\title{
The Regulatory Role of Syntaxin I N-terminal Conformation in Vesicle Priming and Exocytosis
}

\author{
Dissertation \\ zur Erlangung des Doktorgrades \\ der Mathematisch-Naturwissenschaftlichen Fakultäten \\ der Georg-August-Universität zu Göttingen
}

Vorgelegt von

Jong-Cheol Rah

aus Seoul, Korea

Göttingen

2004 
D 7

Referent: Prof. Dr. Erwin Neher

Korreferent: Prof. Dr. Dietrich Gradmann

Tag der mündlichen Prüfung: 3 November 2004 


\section{CONTENTS}

\section{Abstract................................................................ I}

2. Introduction................................................................. 3

2.I Basic function of a neuron and a synapse................................................ 5

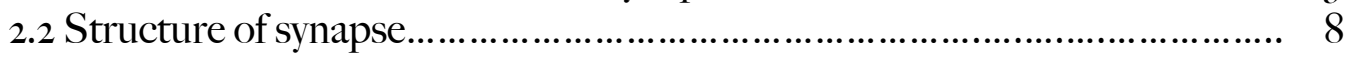

2.2.I Active zones........................................................................ 8

2.2.2 Post-synaptic density.............................................................. IO

2.2.3 Glutamate receptors................................................................ IO

2.3 Vesicle Cycle and Pool Dynamics........................................................... I2

2.4 Release machinery............................................................................. 16

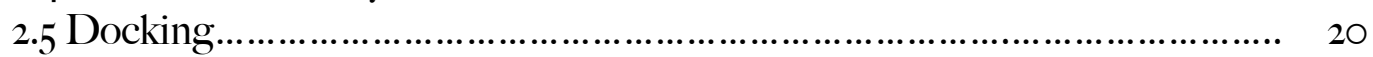

2.6 Priming: Munci8-I, Munciz and RIM.................................................. 2I

2.7 $\mathrm{Ca}^{2+}$ sensing/fusion: synaptotagmins and complexins............................... 25

2.8 Short-term plasticity and vesicle priming............................................ 27

2.9 Specific aim of the study: Syntaxin I-isoforms, structure and function....... 30

3. Experimental Procedure................................................... $\quad 35$

3.I Cell Culture.................................................................................. 36

3.I.I Microisland Hippocampal Culture............................................ 36

3.I.2 Hippocampal Neuron Preparation............................................. 39

3.2 Genotyping................................................................................ 40

3.2.I Genomic DNA Purification...................................................... 40

3.2.2 Genotyping PCR …………………………………….......... 42

3.3 Protein Assays............................................................................. 44

3.3.I Immunoblot Analysis................................................................. 44

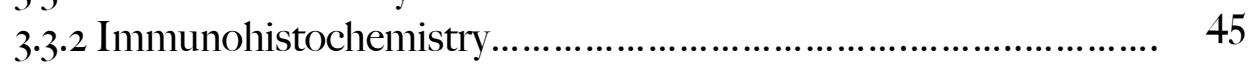

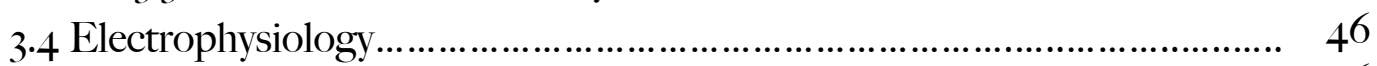

3.4.I Experimental conditions........................................................... 46

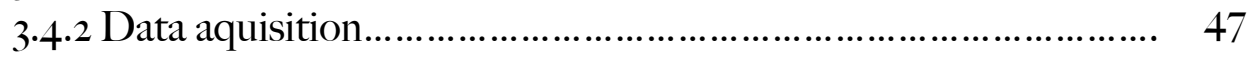

3.4.3 Standard external and internal solutions for electrophysiology.. $\quad 47$

3.4.4 Stimulation protocols and Electrophysiological parameters...... 48

3.4.4-I Evoked response............................................................. 48

3.4.4-2 Determining the size of RRP and PVr.......................... 50

3.4.4-3 Short-term plasticity.................................................. $5^{\mathrm{I}}$

3.4.4-4 Calcium Sensitivity of evoked neurotransmitter release ... $\quad 5^{\mathrm{I}}$

3.4.4-5 Synaptic release probability........................................... 54

3.4.4-6 Spontaneous release.................................................. 55

3.4.4-7 Somatic Calcium Current............................................... $5^{6}$ 
3.4.4-8 Kinetics of sucrose responses.

3.4.4-9 Osmotic pressure-dependent neurotransmitter release.... $\quad 5^{8}$

3.4.4-IO Maximum release rate................................................. $5^{8}$

3.4.4-II Rate of vesicle turn over or refilling of readily releasable pool...

3.4.4-I2 PDBU-induced augmentation...................................... 60

3.5 Experimenter bias minimization.............................................................. 60

3.6 Data display and Statistics.......................................................................... 6I

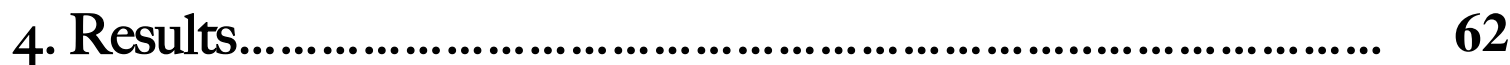

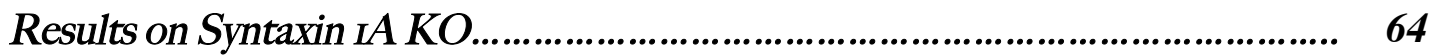

4.I Mutant mouse strain, StxiAKO .............................................................. 64

4.2 Expression of StxiA and StxiB in WT, HZ and KO............................. 64

4.3 Tissue distribution of StxiA and StxiB in hippocampus......................... 67

4.4 Basic Characteristics in release efficacy of stxiA KO............................ 70

4.5 Short-term plasticity characteristics of StxiA KO................................... 72

4.6 Calcium Sensitivity............................................................................. $\quad 75$

4.7 Spontaneous Neurotransmitter Release in stxiA KO............................. $\quad 78$

4.8 Turnover rate of readily releasable pool in stxiA KO.............................. 80

4.9 StxiA KO cells as a control .................................................................... 83

Results on double mutant mice expressing constitutively open conformation

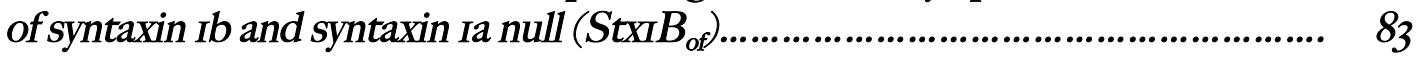

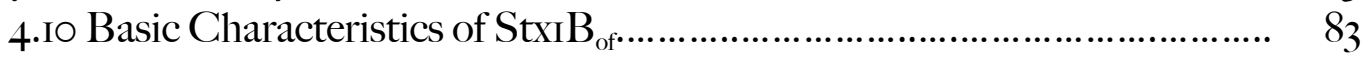

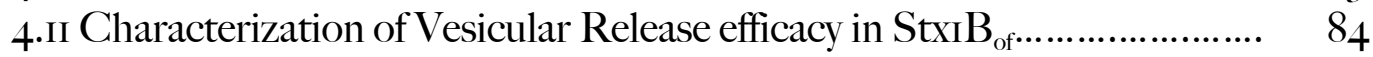

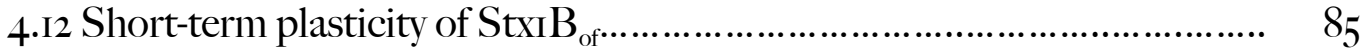

4.I3 Synaptic release probability ................................................................. 88

4.I4 Calcium sensitivity to neurotransmitter release...................................... $\quad 92$

4.I5 Somatic Calcium Current..................................................................... 93

4.I6 Spontaneous Neurotransmitter Release.................................................. 97

4.I7 Neurotransmitter release time course by osmotic pressure...................... I. IOO

4.I8 Neurotransmitter release by smaller osmotic pressure.............................. IO. $\mathrm{IO} 4$

4.I9 Priming rate of vesicles.......................................................................

4.20 Diacyl glycerol/ $\beta$-phorbol ester induced augmentation.......................... I.. I09

5. Discussion................................................................. II5

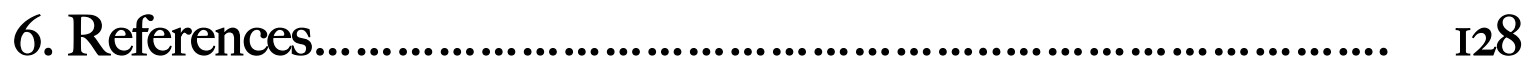




\section{Index of figures and tables}

Figure I Synapses...................................................................................... 5

Figure 2 Model of neuronal SNAREs assembly into the core complex................. 15

Table I Previous studies on SNARE proteins................................................... 19

Figure 3 Model for the role of Munci8-I in membrane fusion................................... 21

Figure 4 Schematic diagram and example of autaptic neuron................................. 38

Table 2 Polymerase chain reaction for genotyping............................................. 43

Figure 5 EPSC, RRP and calculation of vesicular release probability.................. 49

Figure 6 Calculation of EPSC change in various $\mathrm{Ca}^{2+}$ concentrations................... 53

Figure $7 \quad$ Western blotting analysis of StxiA and StxiB ..................................... 66

Figure 8 Distribution of StxiA and StxiB in hippocampus................................. 69

Figure 9 Release efficacy and short-term plasticity of WT and StxKO neurons..... 74

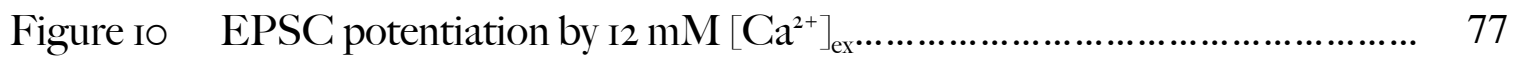

Figure II Spontaneous neurotransmitter release in WT and StxiAKO.................. 79

Figure I2 Turnover rate of readily releasable pool............................................... 82

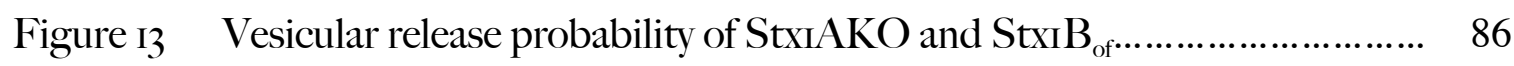

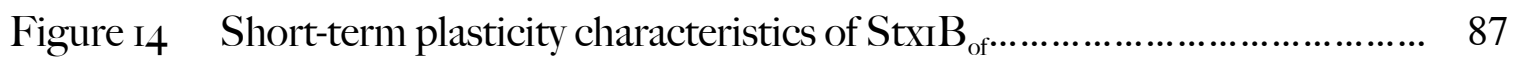

Figure I5 Determining Synaptic release probability as given by the decay of NMDA dependent current in the presence of MK-80I. 
Figure i6 Dependency of EPSC amplitude upon external $\mathrm{Ca}^{2+}$ concentration in $\mathrm{Stxi}_{\text {of }}$ neurons............................................................................... 95

Figure 17 Somatic $\mathrm{Ca}^{2+}$ current comparison between control and $\mathrm{StxIB}_{\text {of }} \ldots \ldots \ldots \ldots \ldots . . . . . \quad 96$

Figure I8 Spontaneous neurotransmitter release efficiency in $\mathrm{StxiB}_{\text {of }}$ neurons........ 99

Figure I9 Time courses of neurotransmitter release by $500 \mathrm{mM}$ sucrose................. 103

Figure 20 Determination of an energy barrier for fusion by released fraction of RRP and maximum released pool unit/s........................................... 107

Figure 2I Steady state amplitude of sucrose response.......................................... 112

Figure 22 Comparison of priming rate between control and $\mathrm{StxiB}_{\text {of }}$ neurons.......... 113

Figure 23 Alternative role of Muncı 3 in vesicle priming/fusion................................. 114 


\section{Abbreviations}

$\begin{array}{ll}\text { AMPA } & \text { Alpha-amino-3-hydroxy-5-methyl-4-isoxazole } \\ \text { propionic acid } \\ \text { ATP } & \text { Adenosine triphosphate } \\ \text { BSA } & \text { Bovine serum albumin } \\ \text { BTCI } & \text { Botulinum toxin CI } \\ \text { C. elegans } & \text { Caenorhabditis elegans } \\ \text { ConA } & \text { Concanavalin A } \\ \text { DAG } & \text { Diacyl glycerol } \\ \text { DMEM } & \text { Dulbecco's Modified Eagle Medium } \\ \text { DNA } & \text { Deoxyribonucleic acid } \\ \text { dNTP } & \text { Deoxyribonucleoside triphosphat } \\ \text { D-AP5 } & \text { D-2-amino-5-phosphonopentanoic acid } \\ \text { Drosophila } & \text { Drosophila melanogaster } \\ \text { EDTA } & \text { Ethylenediaminetetraacetic acid } \\ \text { EPSC } & \text { Excitatory Postsynaptic Current } \\ \text { EtBr } & \text { Ethidium Bromide } \\ \text { FBS } & \text { Fetal Bovine Saline } \\ \text { FUDR } & \text { 2-deoxy-5-fluorouridine } \\ \text { GTP } & \text { Guanine triphosphate } \\ \text { HBSS } & \text { Hanks balanced salt solution } \\ \text { HEPES } & \text { N-2-Hydroxyethylpiperazine-N'-2-ethanesulfonic } \\ & \text { acid } \\ \text { HZ } & \text { Heterozygote } \\ \text { IPSC } & \text { Inhibitory Postsynaptic Current } \\ \text { KA } & \text { kainate } \\ \text { KO } & \text { Knockout } \\ \text { mEPSC } & \text { Miniatur EPSC } \\ \text { mOsm } & \text { Miliosmolar } \\ \text { Munc } & \text { Mammalian Homolog of unc } \\ \text { NBA } & \text { Neurobasal-A-Medium } \\ \text { NBQX } & \text { 6-nitro-7-sulfamobenzo quinoxaline-2,3-dione } \\ \text { NMDA } & \text { N-methyl-D-aspartate } \\ \text { NSF } & \text { N-ethylmaleimide-sensitive fusion protein } \\ \text { PAGE } & \text { Polyacrylamide gel electrophoresis } \\ \text { PCR } & \text { Polymerase chain reaction } \\ \text { PDBU } & \text { 3-phorbol ester dibutyrate } \\ \text { Pvr } & \text { Vesicular release probability } \\ & \end{array}$




\begin{tabular}{|c|c|}
\hline RIM & Rab3 interacting molecule \\
\hline RRP & readily releasable pool \\
\hline SDS & Sodium dodecyl sulfate \\
\hline SEM & Standard Error of the Mean \\
\hline SNAP & Soluble NSF attachment proteins \\
\hline SNAP-25 & Synaptosomal associated protein of $25 \mathrm{kDa}$ \\
\hline SNARE & $\begin{array}{l}\text { Soluble N-ethylmaleimide-sensitive fusion- } \\
\text { attachment protein receptors }\end{array}$ \\
\hline STP & Short-Term Plasticity \\
\hline Stx & Syntaxin \\
\hline $\mathrm{StxIB}_{\text {of }}$ & $\begin{array}{l}\text { Double mutant mice expressing constitutively open } \\
\text { conformation of syntaxin } \mathrm{Ib} \text { and syntaxin Ia null }\end{array}$ \\
\hline Taq & Thermophilus aquaticus \\
\hline TBE & Tris-Borate-EDTA \\
\hline TE & Tris-EDTA \\
\hline TEA & Tetraethyl ammonium \\
\hline TS & Tris-Sodium Chloride buffer \\
\hline t-SNARE & Target membrane SNAREs \\
\hline TTX & Tetrodotoxin \\
\hline Unc & Uncoordinated Movement Mutant \\
\hline VAChT & Vesicular acethyl choline transporter \\
\hline VAMP & Vesicular-associated membrane protein) \\
\hline VGluT & Vesicular glutamate transporter \\
\hline VMAT & Vesicular Monoamine transporter \\
\hline v-SNARE & Vesicular membrane SNAREs \\
\hline W'T & Wild type \\
\hline$\beta-\mathrm{PE}$ & $\beta$-phorbol ester \\
\hline
\end{tabular}


I. Abstract 


\section{ABSTRACT}

During vesicle priming at the central synapse, Syntaxin I, together with SNAP25 and Synaptobrevin, assemble into the synaptic SNARE complex. The formation of SNARE complex is thought to provide the energy needed that enables vesicle exocytosis during $\mathrm{Ca}^{2+}$ triggering step at the presynapse. Among the SNARE proteins, Syntaxin $\mathrm{I}$ is thought to play a special role in regulating the rate of SNARE assembly by adopting two conformations; a closed autoinhibitory conformation in which core complex domain is hindered by N-terminal Habc domain, and an open conformation in which the SNARE motif of syntaxin is open and ready to interact with the other SNAREs.

In the present study we examined the role of the conformational switch of syntaxin I by analyzing synaptic properties of genetically modified mice expressing a mutation that leaves syntaxin I in a constitutively open conformation. In cultured hippocampal neurons, the mutation led to a significant increase in the rate of vesicle priming, supporting the hypothesis that syntaxin I regulates vesicle priming during the SNARE complex assembly process. Surprisingly, we also found that the mutation led to an increase in synaptic release probability, suggesting that the conformation of syntaxin I also regulates vesicle fusion by reducing the energy barrier of vesicle fusion to the plasma membrane. Our data support the idea that the SNARE complex member syntaxin I and its individual conformations are crucial regulators of the efficacy and short-term plasticity of synaptic transmission. 
2. Introduction 


\section{INTRODUCTION}

“Cogito, ergo sum (I think therefore I am)”, René Descartes said in I637, however, his question, "how does thinking stuff keep company with material stuff?" still remains unanswered. How do molecules, which are basically permutations and combinations of atomic moieties, synchronize, in perfect harmony, to create the orchestra of life: actions and reactions, thoughts, emotions, language, learning and memory? If one embarks on the journey to trace the origin of these highly sophisticated functions of the brain that creates the mind, the neuron is unquestionably the final destination.

Neurons are the basic units of nervous system. There are trillions of neurons in human brain and each of these neurons makes upto Iooo connections through their processes with one another. Therefore, neurons are the fundamental building blocks of the network of which the nervous system is composed. A neuron is designed to detect and integrate incoming information to transmit the reacting activity along its process to other neurons in the network so as to maintain a constant ebb and flow of information. 


\section{I Basic Function of a Neuron and a Synapse}

Neurons are strongly compartmentalized and polarized cells. With a few exceptions, a neuron has three major functional and morphological domains: cell body, dendrite and axon. The cell body contains genetic materials and the cytoplasmic organelles like other cells. Dendrites extend from cell body and ramify so as to increase the area of contact with other neurons. These two specialized compartments of the neuron, the cell body and dendrites are the major domains that receive inputs from other neurons. The axon extends, in most cases, much further from the cell body than the dendritic arbor does. Many of the axons are surrounded by an insulating myelin sheath, which facilitates rapid impulse conduction. The axon is responsible for transmitting neural information to other cells. The terminal region
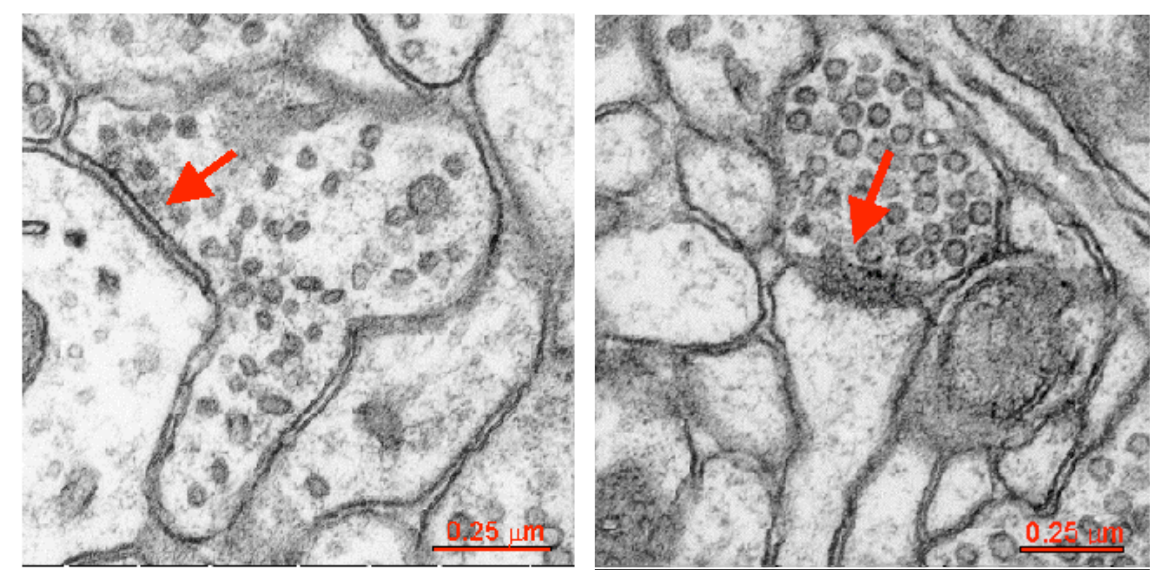

[Figure1] Representative symmetric (presumably inhibitory; left) and asymmetric synapses (presumably excitatory; right). Arrow points to the synapse from the presynaptic side. Adopted from http://synapses.mcg.edu/anatomy/ 
of the axon, where contacts with other cells are made is called presynaptic terminal and is part of a specialized morphological structure called the synapse.

Information is encoded in a neuron by electrical impulses. Synapses serve as the junction of this information flow from one cell to the next, directly in the form of electrical signals (e.g. bidirectional electrical synapses) or transformed as chemical signals via the release of neurotransmitter (e.g. unidirectional excitatory and inhibitory synapses based on the type of transmitter released). These chemical synapses can be morphologically and functionally distinguished: the asymmetric synapses usually carrying excitatory inputs and symmetric synapses carrying inhibitory inputs leading to depolarization and hyperpolarization of the postsynaptic neuron, respectively (figure I).

Most excitable cells have a resting potential in the range of $-60 \mathrm{mV}$ to -90 $\mathrm{mV}$, while equilibrium potentials of the four major ions namely $\mathrm{Na}^{+}, \mathrm{K}^{+}, \mathrm{Ca}^{2+}$ and $\mathrm{Cl}^{-}$of around $+50 \mathrm{mV},-70 \mathrm{mV},+130 \mathrm{mV}$ and $-70 \mathrm{mV}$, respectively along with their asymmetric distributions across the membrane lead to an electrochemical gradient. Thus, in their resting state, cells maintain a constant driving force, in the form of potential difference, for flow of each ion. This resting state of potential difference across the membrane is called polarized state and is maintained primarily by sodium-potassium ATPase, which actively transport three $\mathrm{Na}^{+}$ions out of the cell in 
exchange of two $\mathrm{K}^{+}$ions, thereby establishing a default negative resting membrane potential.

When the neuron receives a large enough stimulus, the sodium conductance increases transiently, due to voltage-dependent gating of $\mathrm{Na}^{+}$channels. These changes in $\mathrm{Na}^{+}$conductance drive the membrane potential toward the $\mathrm{Na}^{+}$ equilibrium potential which depolarizes the membrane, and is followed by an increase in $\mathrm{K}^{+}$conductance that later drives the membrane potential back to its resting potential. At this point the action potential propagates along axons by longitudinal spread of current and finally reaches the point of functional contact, the synapse.

When an action potential invades the axon's presynaptic terminal, depolarization activates voltage-gated $\mathrm{Ca}^{2+}$ channels (mainly $\mathrm{P} / \mathrm{Q}-$ and $\mathrm{N}$-type). $\mathrm{Ca}^{2+}$ ions move inward and trigger the synaptic vesicles to fuse with presynaptic plasma membrane so as to release their neurotransmitter contents into the synaptic cleft. Released neurotransmitters, then, diffuse and subsequently bind to specific receptors at the postsynapse to evoke excitatory or inhibitory postsynaptic potentials (EPSP/ IPSP). Majority of inter-neuronal communication relies on the use of this chemical transmission. Through this process, an action potential in the presynaptic terminal propagates to neighboring cell in a few milliseconds. 


\subsection{Structure of Synapse}

To achieve efficient synaptic transmission, exocytosis of neurotransmitter is restricted to specialized structures known as 'active zones' in the presynaptic terminal, which targets directly upon specialized 'post synaptic density'.

\subsection{Active Zones}

One of the structural studies using electron microscope tomography at a model synapse, the frog neuromuscular junction elegantly showed the arrangement and associations of structural components of compact organelle into an active zone. This study showed that it is approximately $\mathrm{I}-2 \mu \mathrm{m}$ long, $75 \mathrm{~nm}$ wide and typically extends $5^{\circ-75} \mathrm{~nm}$ from the presynaptic membrane into the cytoplasm. The three main components of this structure are known as beams, ribs and pegs. The ribs extend orthogonally into the ridge's long axis and form approximately 7 -I2 connections to docked vesicles located on each flank of the ridge (Harlow et al., 200I; Rosenmund et al., 2003). This might help to dock the vesicles and tether the channels for efficient $\mathrm{Ca}^{2+}$ supply, which is essential for fast turn on and off of exocytosis. Because intracellular $\mathrm{Ca}^{2+}$ concentration $\left(\left[\mathrm{Ca}^{2+}\right]_{\mathrm{i}}\right)$ is maintained in very low (about Ioo $\mathrm{nM}$ ) in comparison to external high extracellular $\mathrm{Ca}^{2+}$ concentration $\left(\left[\mathrm{Ca}^{2+}\right]_{\mathrm{ex}}\right.$ : millimolar $), \mathrm{Ca}^{2+}$ channel opening causes large changes in $\left[\mathrm{Ca}^{2+}\right]_{\mathrm{i}}$. Proximity of vesicles from the $\mathrm{Ca}^{2+}$ channels have an influence on the probability as well as speed of release, one factor determining these two parameters being the $\mathrm{Ca}^{2+}$ 
cooperativity. For the vesicles located far away from the $\mathrm{Ca}^{2+}$ channels would sense apparent changes in presynaptic $\mathrm{Ca}^{2+}$ concentration to a smaller extent and since release probability is proportional to the fourth power of the $\mathrm{Ca}^{2+}$ concentration (Yoshihara and Littleton, 2002) so accordingly, these distant vesicles will have much lower release probability as well as a longer lasting time course of release compared to their proximal counterparts.

Some proteins are exclusively present at the active zone. Bassoon has been reported to be involved in the tethering of vesicles based on the studies on mice lacking Bassoon, which lead to free floating clustered ribbon in retinal neuron (Dick et al., 2003). Munci3 and RIM are known to have a critical role in the priming process but not in the docking or recruiting of vesicles (Augustin et al., I999; Koushika et al., 200Ia). $\alpha$-liprins bind to receptor tyrosine kinase and regulates the differentiation of synaptic terminal (Serra-Pages et al., I998; Zhen and Jin, I999). CAST (cytometrix at the active zone (CAZ) - associated structural protein) is known to form a protein network with other CAZ associated proteins including RIM, Muncizs and Bassoon at the active zone (Ohtsuka et al., 2002) and Piccolo is another CAZ-associated protein but their physiological function remains not clear (Meyer and Rosenmund, unpublished). How these active zone proteins, CAST and Piccolo contribute to the coordination of presynaptic signal remains unknown. 


\subsubsection{Postsynaptic Density}

The structure known as the postsynaptic density (PSD) is located at the opposite side of the active zone across the synaptic cleft. It is here, where specific sets of molecules including each glutamate receptor and other PSD associated proteins are located. In addition to glutamate receptors, this postsynaptic specialization of neurons, is marked by the presence of scaffolding proteins, like PSD-95 and organization of other membrane proteins such as adhesion molecules, receptor tyrosine kinases, and ion channels.

\subsubsection{Glutamate Receptors}

Glutamate receptors can be divided into two categories, ionotropic and metabotropic. Ionotropic receptors are classified into AMPA (alpha-amino-3hydroxy-5-methyl-4-isoxazole propionic acid) receptor subclass, NMDA (N-methylD-aspartate) receptor subclass, and KA (kainate) receptor subclass. Metabotropic receptors, consists of 3 classes (class I through class III) and 8 genes (mGluRI through mGluR8), and they are metabotropic because they are linked by G-proteins to cytoplasmic enzymes and further classified depending on the signal transduction pathway.

The predominant receptor class for fast excitatory synaptic transmission by glutamate is the AMPA receptor. These are widely spread throughout the central nervous system, and require coassembly of 4 subunits (GluRI through GluR4). 
NBQX (6-nitro-7-sulfamobenzo quinoxaline-2,3-dione) and 2,3-benzodiazepine are known to specifically block these receptors. Subunits of the GluR 5 through GluR7 have been found to coassemble with KA receptor subunit KAI or KA2 to form the KA receptor. KA receptors have relatively low binding affinity to glutamate but only two pharmacological drugs, concanavalin A (ConA) and cycothiazide, can distinguish KA receptors from AMPA receptors. These non-NMDA receptors gate cation ions with relatively low conductance $(<<20 \mathrm{pS})$ that are permeable to both $\mathrm{Na}^{+}$and $\mathrm{K}^{+}$but usually not permeable to $\mathrm{Ca}^{2+}$.

NMDA receptors are very tightly regulated. Since they have two distinguished agonist site for glutamate and glycine and their voltage-dependent blocking by extracellular $\mathrm{Mg}^{2+}$. $\mathrm{Mg}^{2+}$ tightly binds to NMDA channel at the resting potential and is expelled from the channel by depolarization. Thus, the activation of NMDA channel requires glutamate, glycine and depolarization of the cell. The receptor controls cation channels of high conductance $(5 \circ \mathrm{pC})$ that is permeable to $\mathrm{Ca}^{2+}$ as well as $\mathrm{Na}^{+}$and $\mathrm{K}^{+}$. D-2-amino-5-phosphonopentanoic acid (D-AP5) is the competitive antagonist of NMDA receptor, and MK-80I can selectively block activated NMDA receptors (for further reading, see (Kandel et al., 2000; Siegel et al., 1999)) 


\subsection{Vesicle Cycle and Pool Dynamics}

The synaptic cleft is too wide to be traversed by current; instead, neurotransmitters stored in synaptic vesicles are secreted to induce postsynaptic excitation. Synaptic vesicles are actively filled with neurotransmitters by 4 different specialized transporter proteins: a monoamine transporter for all biogenic amines (called VMAT), for GABA and glycine, for acetylcholine (called VAChT), and for glutamate (called VGluT). These transporters are basically vacuolar proton pumps, which cause an electrochemical gradient across the synaptic vesicular membrane. This electrochemical gradient across the membrane is energetically coupled to transport of neurotransmitter into the vesicles. The vesicles once filled with transmitters are, then, moved to the active zone of the presynaptic terminal, but exact mechanism is not yet understood (See part 2.5).

Once vesicles arrive at the active zone, vesicles become attach to the plasma membrane. This attachment process is called, 'docking' (see part 2.5). Then, docked vesicles undergo an ATP-dependent pre-fusion reaction called 'priming' (see part 2.6) to bring vesicles to a fusion competent state. Vesicle priming is considered to be equivalent to the assembly of the release apparatus that catalyzes the fusion of the vesicle with the plasma membrane when $\mathrm{Ca}^{2+}$ influx initiates release. When an action potential arrives at the presynaptic terminal, $\mathrm{Ca}^{2+}$ triggers the completion of fusion. This process requires the binding of multiple $\mathrm{Ca}^{2+}$ ions to synaptic $\mathrm{Ca}^{2+}$ binding sites including synaptotagmin, but it occurs in a very rapid manner. 
After exocytosis, synaptic vesicles are rapidly retrieved by endocytosis, most likely via clathrin coated pits. These endocytosed vesicles then get rid of the coats, and are recycled to the interior of the synaptic nerve terminal. Some of the empty vesicles are either refilled immediately with neurotransmitter or may pass through an endoplasmic reticulum while others may be replaced by a rapid endocytosis process called 'kiss and run' (for review (Südhof and Scheller, 200I)).

Readily releasable vesicles cover only a small fraction of the total vesicle population. The pool constitutes those vesicles that are primed and immediately available for release, defined as the readily releasable pool (RRP). In synapses of cultured hippocampal neurons, the RRP comprises only five to nine synaptic vesicles per each synapse (Murthy and Stevens, I999). However, when release is triggered, usually only a single vesicle of the RRP fuses per synapse, although all of the vesicles in the RRP appear to be ready for fusion. The low release probability of each individual vesicle probably restricts exocytosis to a single vesicle. The overall synaptic release probability is the sum of the individual vesicular release probabilities. Consistently, the number of vesicles in the RRP appears to be a major determinant of the synaptic release probability (Dobrunz and Stevens, 1997).

When synapses are stimulated extensively, the RRP will be depleted quickly, and new population of vesicles will replenish the RRP. The population of vesicles 
contributing to this replenishment is known as 'reserved pool', which in the hippocampal neuron is estimated to be around $17-20$ vesicles (Murthy and Stevens, I999). These two pools, RRP and reserved pool can be stained with FMI-43 when synapses are stimulated extensively and these are named as the 'recycling pool'.

Rather surprisingly, the hippocampal synapses were reported to have about 200 synaptic vesicles in a morphological study (Schikorski and Stevens, 1997), thus majority vesicles are reluctant to fuse under normal conditions. This fraction of the entire vesicular population is referred to as the 'resting pool' (for review (Südhof, 2000)) 


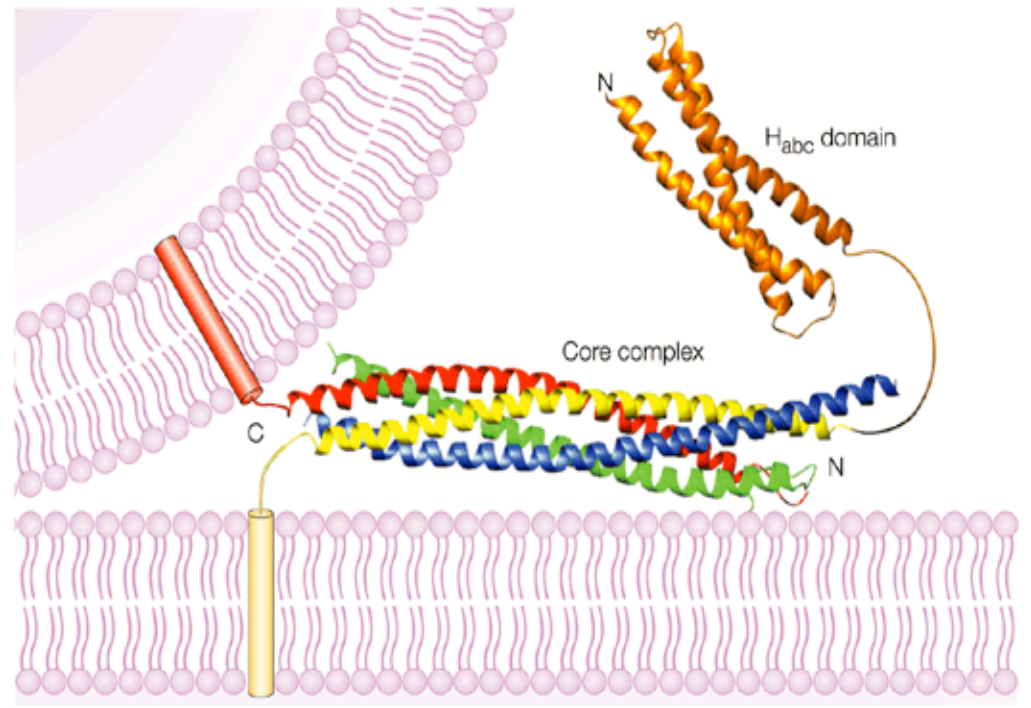

[Figure 2] Model of the neuronal SNAREs assembled into the core complex. The ribbon diagrams represent the crystal structure of the core complex The Habc domain is shown in orange and the SNARE (SNAP receptor) motifs are colored as follows: synaptobrevin, red; syntaxin 1, yellow; SNAP25, blue; SNAP25 carboxyl terminus, green. The cylinders represent the transmembrane regions of synaptobrevin and syntaxin 1 , which are inserted into the synaptic vesicle and plasma membranes, respectively. The curved lines represent short sequences that connect the SNARE motifs and the transmembrane regions, as well as the linker region between the Habc domain and the SNARE motif of syntaxin 1. Adopted from (Rizo and Südhof, 2002) 


\subsection{Release machinery}

How exactly do fundamental processes like vesicle targeting to the active zones and fusion events occur, are still largely left to be elucidated. However, there is compelling evidence to support the hypothesis that the SNARE (soluble Nethylmaleimide-sensitive fusion-attachment protein receptors) complexes drive membrane fusion.

The current hypothesis poses that the selective docking of a vesicle with the appropriate target membrane is mediated by the formation of a complex between the vesicle membrane protein (v-SNARE: synaptobrevin or VAMP) and corresponding target membrane proteins (t-SNAREs: syntaxin and SNAP-25 (synaptosomal-associated protein of $25 \mathrm{kDa})$ ). These proteins zipper up from their N-termini towards the C-termini so as to form a stable trans-SNARE complex, thereby, overcoming counteracting electrostatic forces and leading to the close apposition of opposing vesicle and target lipid bilayers [figrue2]. (for review see Fasshauer, 2003 or Jahn et al., 2003).

SNARE motifs, a moderately conserved stretch of 60 to 70 amino acids, at C-terminal domain of syntaxin I, the cytoplasmic domain of synaptobrevin and Nand C-terminal domains of SNAP-25 assemble spontaneously into an extraordinarily stable complex of four-helix bundles. It has been reported that at the 
center of the SNARE complex, there are conserved Leucine-zipper-like layers embedded with ionic layers consisting of three glutamine and one arginine residues contributed from each SNARE motif alpha-helix (Sutton et al., I998).

Several lines of evidence utilizing SNARE protein-targeted clostidial neurotoxins and genetically modified organisms have shown that the SNARE complex is absolutely necessary for efficient membrane fusion [Table I]. Furthermore, in vitro reconstitution experiments have shown that SNAREs are the minimally sufficient fusion machinery, by demonstrating that SNAREs reconstituted in separate artificial lipid bilayer vesicles lead to spontaneous fusion under physiological condition (Weber et al., I998).

However, although all of the mutations or truncations in SNARE proteins have led to complete ablation of action-potential induced evoked responses, spontaneous release was still observed in some cases, such as SNAP-25 null mice and synaptobrevin knock out mice (Schoch et al., 200I; Washbourne et al., 2002), Table I. These findings suggest that perhaps SNARE complex is not necessary for all kinds of neurotransmitter release, or that spontaneous neurotransmitter release uses a different mechanism other than the SNARE complex formation. Another explanation is that the observed releases in theses null mouse lines might be originated from the isoforms of eliminated proteins. For example, in the case of study with SNAP-25 knock-out mouse, SNAP-23 may compensate the lack of 
SNAP-25. In fact, western blot analysis in the lack of SNAP-25 in this study showed comparably increased expression of SNAP-23 in both heterozygotes and knock-out mice, even though it has not been discussed in detail (Washbourne et al., 2002). In case of synaptobrevin-2 knockout study, however, synaptobrevin I and cellubrevin were examined and considered carefully as a potential substitute for the protein. Furthermore, in this case, IO\% of RRP remained. Thus, it is less likely that the release is purely the outcome of compensation, because the compensation under detection limit of western blotting does not sufficiently explain the remaining Io\% of RRP (Schoch et al., 200I). A more realistic explanation is that the fusion reaction can still happen without SNARE complex, but SNARE complex is needed because it critically catalyzes an increase in the rate of membrane priming and fusion (Schoch et al., 200I).

In vivo, the SNARE proteins are not the only proteins involved in neurotransmitter release but numerous proteins, which do not function as fusion factors, are reported to mediate or facilitate fusion events. These proteins include NSF (N-ethylmaleimide-sensitive fusion protein), SNAP (soluble NSF attachment proteins). NSF is the ATPase chaperone which disassemble the stable SNARE complex in conjunction with the soluble NSF attachment proteins (SNAPs) as cofactors (Otto et al., I997). Proteins such as synaptotagmin I and complexins are reported to be crucial mediator for $\mathrm{Ca}^{2+}$ mediated exocytosis. Muncizs and Munc I8 are known as key regulator for conformation of syntaxin I (see part 2.6). 


\section{[Table 1] Previous studies on SNARE proteins}

\begin{tabular}{|c|c|c|c|c|}
\hline \multirow{4}{*}{$\begin{array}{l}\text { Synapto- } \\
\text { brevin }\end{array}$} & $\begin{array}{l}\text { Tetanus } \\
\text { toxin }\end{array}$ & $\begin{array}{l}\text { Abolished evoked, but not } \\
\text { spontaneous synaptic } \\
\text { vesicle release }\end{array}$ & $\begin{array}{c}\text { Normal } \\
\text { development }\end{array}$ & $\begin{array}{c}\text { (Sweeney et al., } \\
\text { I995) }\end{array}$ \\
\hline & C. elegans & $\begin{array}{c}\text { Impaired cholinergic } \\
\text { transmission; aldicarb } \\
\text { resistance }\end{array}$ & $\begin{array}{c}\text { Die after } \\
\text { embryogenesis }\end{array}$ & (Nonet et al., I998) \\
\hline & Drosophila & $\begin{array}{l}\text { Abolished evoked, and } 75 \% \\
\text { reduced spontaneous, } \\
\text { synaptic vesicle release }\end{array}$ & $\begin{array}{c}\text { Lethal; } \\
\text { paralysis; } \\
\text { normal } \\
\text { morphology }\end{array}$ & $\begin{array}{c}\text { (Deitcher et al., } \\
\text { I998) }\end{array}$ \\
\hline & Mice & $\begin{array}{l}\text { Abolished evoked, and } 85 \% \\
\text { reduced spontaneous, } \\
\text { synaptic vesicle release; } 90 \% \\
\text { reduced RRP }\end{array}$ & $\begin{array}{l}\text { Lethal; die } \\
\text { immediately } \\
\text { after birth }\end{array}$ & $\begin{array}{l}\text { (Schoch et al., } \\
\text { 200I) }\end{array}$ \\
\hline \multirow{5}{*}{ SNAP-25 } & $\begin{array}{l}\text { Botulinum } \\
\text { toxin } \mathrm{A} / \mathrm{E}\end{array}$ & $\begin{array}{c}\text { Abolished SNAP-25, } \\
\text { overcome by increased } \\
\mathrm{Ca}^{2+} ; \text { decreased } \mathrm{Ca}^{2+} \\
\text { sensitivity to release }\end{array}$ & & (Binz et al., I994) \\
\hline & $\begin{array}{c}\text { Antisense } \\
\text { oligoneucleo } \\
\text { tide } \\
\end{array}$ & $\begin{array}{c}\text { Inhibited axonal growth, } \\
\text { synaptogenesis }\end{array}$ & & $\begin{array}{l}\text { (Osen-Sand et al., } \\
\text { I993; Osen-Sand et } \\
\text { al., I996) }\end{array}$ \\
\hline & $\begin{array}{c}\text { Botulinum } \\
\text { toxin A }\end{array}$ & Reduced neurite outgrowth & & $\begin{array}{c}\text { (Morihara et al., } \\
\text { I999) }\end{array}$ \\
\hline & $\begin{array}{l}\text { Drosophila } \\
\text {-temperatur } \\
\text { e sensitive }\end{array}$ & $\begin{array}{c}\text { Reduced SNAP-25 reduced } \\
\text { evoked; normal miniature } \\
\text { vesicular release }\end{array}$ & & (Rao et al., 200I) \\
\hline & Mice & $\begin{array}{c}\text { Abolished EPSC; still have } \\
\text { mEPSC with bigger } \\
\text { amplitude } \\
\end{array}$ & $\begin{array}{l}\text { Embryonic } \\
\text { lethal }\end{array}$ & $\begin{array}{l}\text { (Washbourne et } \\
\text { al., 2002) }\end{array}$ \\
\hline \multirow{3}{*}{ Syntaxin } & $\begin{array}{c}\text { Botulinum } \\
\text { toxin } \mathrm{CI}_{\mathrm{I}} \\
\end{array}$ & $\begin{array}{c}\text { Blocked } \\
\text { neurotransmitterelease }\end{array}$ & & (Blasi et al., I993) \\
\hline & $\begin{array}{l}\text { Syntaxin } \\
\text { fragment/ } \\
\text { Anti- } \\
\text { syntaxin } \\
\text { antibody }\end{array}$ & $\begin{array}{c}\text { Depression in } \mathrm{Ca}^{2+}- \\
\text { dependent transmission }\end{array}$ & & $\begin{array}{l}\text { (Bennett et al., } \\
\text { I993) }\end{array}$ \\
\hline & Drosophila & $\begin{array}{l}\text { Complete failure in evoked, } \\
\text { very rare spontaneous } \\
\text { transmitter release }\end{array}$ & $\begin{array}{c}\text { Abnormal } \\
\text { development } \\
\text { (cuticle/ gut/ } \\
\text { yolk digestion) }\end{array}$ & $\begin{array}{l}\text { (Broadie et al., } \\
\text { I995; Schulze et al., } \\
\text { I995) }\end{array}$ \\
\hline
\end{tabular}




\subsection{Docking: Rab3 proteins}

Little is known about how the neurotransmitter vesicles get to have initial contact with plasma membrane (docking). Rab proteins, which are a family of Rasrelated small GTPases are proposed to play the main role in membrane docking. When bound to membranes, Rab proteins operate as molecular switches that are active in the GTP-bound form and inactive in the GDP-bound form. The likely function of active Rab3 is the recruitment of a variety of proteins such as rabphilin, RIM (rab3 interacting molecule), etc. that bridge the membranes of the active zone. However, recent studies by Schlüter et al. showed that quadruple knock-out of $\mathrm{Rab}_{3} \mathrm{~A}, \mathrm{~B}, \mathrm{C}, \mathrm{D}$ led to $30 \%$ decrease in evoked responses due to reduced release probability without significant changes in spontaneous or sucrose-evoked release (Schluter et al., 2004). This finding suggests that Rab3s are essential for $\mathrm{Ca}^{2+}$ triggered neurotransmitter release but unlikely for vesicular docking.

Recently, the possible role of Muncı8 in vesicle docking is suggested, based on the finding of defects in vesicle docking in null mutant C.elegans and mouse chromaffin cells (Voets et al., 200I; Weimer et al., 2003b). 


\subsection{Priming: Munci8-I, Munci3 and RIM}

SNARE proteins are not always ready to form, since syntaxin I exhibits a closed conformation. Release of syntaxin I into an open conformation is believed to be required to allow form the SNARE complex (Dulubova et al., I999; Munson and Hughson, 2002). Syntaxin contains N-terminal Habc domain, linker domain, SNARE motif and C-terminal transmembrane domain. The N-terminal Habc
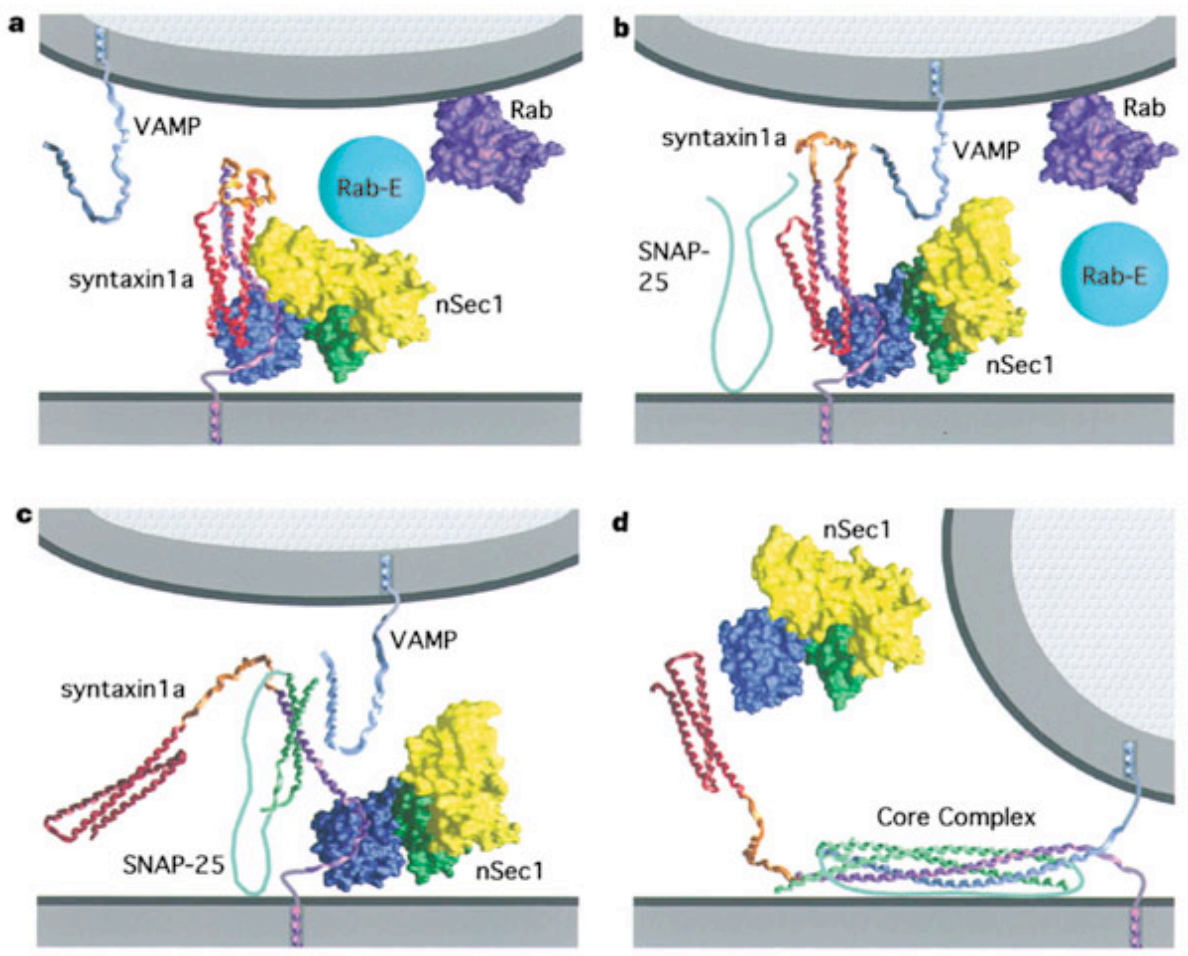

[Figure 3] Model for the role of Munc 18-1(nSec1 in figure) in membrane fusion. a, nSec1 bound to closed conformation of syntaxin 1a. Possibly through a Rab or Rab effector protein (not appeared in this figure). b, Munc13-1 and RIM induces a conformational change in nSec1 and causes the loss of contacts with syntaxin 1a. Exposed residues of $\mathrm{H} 3 \mathrm{a}$ interact with SNAP-25, initiating the formation of the core complex. c, The syntaxin Habc domain moves away from the $\mathrm{H} 3$ region of syntaxin 1a. The SNAP-25 helices propagate towards the C-terminus, and VAMP begins to bind to the helical $\mathrm{H} 3 \mathrm{a}$ region. $\mathbf{d}$, The nucleated VAMP-SNAP-25-syntaxin 1a helices assemble rapidly and complete the long straight helical bundle structure (SNARE complex). Formation of SNARE might then promote membrane fusion. Adopted with modification from (Misura et al., 2000) 
domain is essential for the binding to its interacting partners including Muncr3, Munc I8, and N-type $\mathrm{Ca}^{2+}$ channel (Bennett et al., 1992; Betz et al., 1997; Hata et al., 1993), and reversibly folds back upon the SNARE motif (Dulubova et al., I999; Fernandez et al., 1998). NMR studies on Habc domain showed three long alphahelices in a twisted left-handed up-down bundles (Fernandez et al., 1998). The linker domain of syntaxin I is known to be highly flexible and plays a crucial role in determining a conformation of syntaxin mainly based on the finding that double point mutation of the linker domain (Li65, Ei66) led to fixed open-conformation of syntaxin (Dulubova et al., 1999; Margittai et al., 2003a).

The closed conformation of syntaxin I is believed to be stabilized by Munci8I. Single-molecule fluorescence resonance energy transfer study on the conformation of free syntaxin showed dynamic equilibrium between closed and open conformation with $0.8 \mathrm{~ms}$ of relaxation time, explaining the requirement of regulatory proteins to arrest in one conformational state (Margittai et al., 2003b). Munci8-I is a hydrophilic, 60-70 kDa polypeptide that was first discovered in $C$. elegans for an uncoordinated phenotype named Unc-I8 (Brenner, 1974) and rediscovered in yeast as sec as a gene involved in the yeast secretory pathway (Novick et al., I980). It is generally believed that the role of Munc I8 is to bind to the closed conformation of syntaxin and compete with SNARE complex formation. These consensus based on the finding that the binding of syntaxin to other SNARE partners preclude their interaction with Munc I 8 and that Munc I 8 binds selectively 
to closed form of syntaxin I (Dulubova et al., I999; Yang et al., 2000). This hypothesis is supported by the findings in Drosophila, that syntaxin mutations that eliminate ROP (Munc-I8 orthologue in Drosophila) binding display increased neurotransmitter release, suggesting that ROP negatively regulates neurosecretion through its interaction with syntaxin (Wu et al., I999).

Unexpectedly, the lack of Munci8-I led to complete failure of neurotransmitter release in mammalian system (Verhage et al., 2000). Furthermore, overexpression of Munc I8 in chromaffin cells induced increased membrane fusion (Voets et al., 200I), showing positive role in fusion process for Munci8-I. Also, recently evidence that expression of open-mutation syntaxin I cannot bypass the requirement for unci8 (Weimer et al., 2003a), strongly suggests that the role of Munci8 is not only assist the conformation of syntaxin. Therefore, although it is clear that Munc I8-I plays an essential role in neurotransmitter release, the general physiological role of Munc I8 is not yet clear.

Given that Munci8 is involved in stabilizing the closed conformation of syntaxin I, how can syntaxin be re-activated? Munciz is the strongest candidate for releasing syntaxin out of the lock in by Munc 18 , by switching the conformation of syntaxin I to the Munci8-binding independent open conformation, based on following findings. First, Munciz localizes selectively in active zone. Second, studies in Drosophila, C. elegans, and mice suggest that Munciz functions at a post- 
docking step of exocytosis, most likely during synaptic vesicle priming (Aravamudan et al., 1999; Augustin et al., 1999; Brose et al., 1995; Maruyama and Brenner, I99I). Third, from in vitro binding assay, the carboxyl terminus of Muncr 3 binds competitively with Munc I8 to amino terminal region of syntaxin I, sharing binding domain with Munc I8-I (Betz et al., I997; Sassa et al., I999). Finally, and most importantly, overexpression of constitutively open form of syntaxin rescued the phenotype of unc-I3 null C. elegans, which strongly suggesting that Munciz is the protein to switch conformation of syntaxin I (Richmond et al., 200I).

RIM is another molecule necessary for switching the conformation of syntaxin I. RIMI and RIM2 were found in the search for putative effector molecule of rab3. RIMs bind to rab3 via their zinc finger domains, and via its PDZ domain and $\mathrm{C}_{2}$ domain it can bind phospholipids and $\mathrm{Ca}^{2+}$. Thus, hypothetically, by means of domain structure to function prediction, RIM could bring the vesicles to dock to the membrane.

However, it is not likely that this is the main role of RIMs, since deletion of one of the isoforms, RIMI, caused a much more severe phenotype (severe reduction in neurotransmitter release) than rab3 deletion (Koushika et al., 200Ia). RIMI seems to have its main role as a critical helper for Muncız because $60 \%$ reduction of Munci3 is reported in RIMı null mice (Schoch et al., 2002). Moreover, disruption of the interaction between RIMi and Munci3-I led to a loss of fusion-competent 
synaptic vesicles, creating a similar phenotype to Munciz-I-deficient neurons, suggesting that RIMI is necessary for Munc13-I to carry out its function in priming (Betz et al., 200I). Correlatively, the phenotype of $C$. elegans lacking RIM can also be rescued by overexpression of syntaxin I (Koushika et al., 200Ia).

\section{7 $\mathrm{Ca}^{2+}$ sensing/fusion: synaptotagmins and complexins}

Once the SNARE complex is formed, neurotransmitter release is regulated by the increment of intracellular calcium concentration $\left(\left[\mathrm{Ca}^{2+}\right]_{\mathrm{i}}\right)$. When an action potential arrives at the presynaptic terminal, high-voltage activated P/Q- and N-type $\mathrm{Ca}^{2+}$ channels open, and $\left[\mathrm{Ca}^{2+}\right]_{\mathrm{i}}$ increases to IO-2O mM, a concentration that triggers vesicular fusion with a I00-200 ms time delay. To meet this time delay, $\mathrm{Ca}^{2+}$ channels must be localized in close proximity to the release machinery, and need a very efficient, but low affinity $\mathrm{Ca}^{2+}$ sensor. Synaptotagmin I is the most attractive candidate since it is a highly conserved synaptic vesicle protein, and binds calcium at physiological concentrations in a complex with negatively charged phospholipids (Brose et al., 1992). Recently, in a reconstitution study involving synaptotagmin and SNAREs, synaptotagmin enhanced both the rate and extent of fusion by binding anionic phospholipids in $\mathrm{Ca}^{2+}$-dependent manner (Tucker et al., 2004). Furthermore, the lack of synaptotagmin I led to dramatic deficits in $\mathrm{Ca}^{2+}$ triggered synchronous neurotransmitter release but not in asynchronous or $\mathrm{Ca}^{2+}$-independent release, strongly suggesting that synaptotagmin I is the major low affinity $\mathrm{Ca}^{2+}$ 
sensor which mediates $\mathrm{Ca}^{2+}$ regulation of synchronous neurotransmitter release in hippocampal neurons (Geppert et al., I994). Most synaptotagmins bind $\mathrm{Ca}^{2+}$ ions through their two C2 domains, which causes secondary acidic phospholipids and/or SNARE protein binding. When a positively charged amino acid residue surrounding the $\mathrm{Ca}^{2+}$ binding domain in the $\mathrm{C}_{2} \mathrm{~A}$ region was mutated to alter $\mathrm{Ca}^{2+}$ binding affinity without affecting three-dimensional structure of $\mathrm{Ca}^{2+}$ binding site, the $\mathrm{Ca}^{2+}$ sensitivity of neurotransmitter release was decreased twofold, but spontaneous release or the size of the RRP were not affected (Fernandez-Chacon et al., 200I). On the other hand, mutating the equivalent amino acid residue in the $\mathrm{C}_{2} \mathrm{~B}$ domain $\left(\mathrm{K}_{3} 66 \mathrm{Q}\right)$ caused no change in $\mathrm{Ca}^{2+}$-dependent properties in syntaptotagmini or evoked transmitter release. Instead, double point mutation in two serial amino acid residues in polybasic domain of $\mathrm{C}_{2} \mathrm{~B}\left(\mathrm{~K}_{32} 6,327 \mathrm{~A}\right)$ showed essentially the same phenotype of $\mathrm{R}_{233} \mathrm{Q}$ mutation in $\mathrm{C}_{2} \mathrm{~A}$ domain ( $\mathrm{Li}$ et al., unpublished), showing that these two $\mathrm{C}_{2}$ domains have. Results described so far indicate that synaptotagmin $\mathrm{I}$ is indeed a $\mathrm{Ca}^{2+}$ sensor and it mediates $\mathrm{Ca}^{2+}$ dependent, rapid, synchronous neurotransmitter exocytosis through its $\mathrm{C}_{2} \mathrm{~A}$ and $\mathrm{C}_{2} \mathrm{~B}$ domain.

Complexin I and II, the abundant proteins in the central synapse, are known to bind to the fully assembled SNARE complex and enhance $\mathrm{Ca}^{2+}$ sensitivity (Pabst et al., 2000). In double deficient mice, the synchronous component of evoked response 
was selectively impaired, while priming and $\mathrm{Ca}^{2+}$ independent neurotransmitter release were unaffected (Reim et al., 200I).

\subsection{Short-term plasticity}

Information within neurons is encoded by spike train patterns. At the synapse, these incoming action potentials are transmitted to the receiving neuron by the initiation of neurotransmitter release via fusion of vesicles with the plasma membrane. In response to stimulation at high frequencies, synapses can undergo profound changes in strength, which can be even greater than Io fold increase or decrease, depending on types of synapses. The efficacy of neurotransmitter release represents the major determinant of how reliable information cross the synapse.

The changes in efficacy of synapses, whether transient or permanent, is referred as synaptic plasticity. Transient or short-term plasticity (STP) critically depends on the rate and duration of incoming action potentials trains and on other factors that may be variable for each presynaptic neuron and each synapse, such as the initial release probability, the number of fusion competent, primed vesicles per synapse.

Various forms of STP, such as short term depression, frequency facilitation, and augmentation govern processes as diverse as sound localization, the generation of oscillatory brain activity, cortical gain control and perhaps many others, even the 
terminals made by the same axon on different target cells can show very different short-term plasticity characteristics. Thus it is generally accepted that STP is of central importance for information processing in the brain.

It has long been known that $\mathrm{Ca}^{2+}$ ions are the mediators of most forms of short-term plasticity. The function and localization of presynaptic $\mathrm{Ca}^{2+}$ channels as well as $\mathrm{Ca}^{2+}$ buffers are major contributors to short-term plasticity, because they influence the presynaptic $\mathrm{Ca}^{2+}$ dynamics.

As actual functional presynaptic changes during STP, two key determinants have been suggested: Residual $\mathrm{Ca}^{2+}$ and pool depletion. Residual $\mathrm{Ca}^{2+}$ hypothesis can explain the facilitation factor during the repetitive stimuli. Since rapid shut off of exocytosis is achieved by rapid removal of $\mathrm{Ca}^{2+}$ from the site of action by various mechanisms i.c. diffusion, $\mathrm{Ca}^{2+}$ buffering etc., a second stimulus can be influenced by the residual calcium left over from the first $\mathrm{Ca}^{2+}$ influx.

The pool depletion factor is mainly governed by vesicle priming rate and the vesicular release probability. Because the number of primed fusion competent vesicles is small, high release probability synapses exhaust the number of available vesicles within a few action potentials. Therefore, replacement of used vesicles with newly primed ones and adaptation of vesicle priming rates during STP will strongly influence how synaptic responses are maintained during trains of action potentials.

As the number of RRP is the limiting factor for short-term plasticity, it valid predicted that the priming factors such as Muncizs and RIMs play crucial roles. 
Munci3-I and -2, especially determine the patterns of short-term plasticity. While Munci3-I mediates synaptic depression, Munci3-2 causes augmentation by a $\mathrm{Ca}^{2+}-$ dependent increase in release probability and RRP size in hippocampal neurons (Rosenmund et al., 2002). In addition, the lack of RIMI causes short-term facilitation by decreasing vesicular release probability. 


\subsection{Specific aim of the study: Syntaxini- isoforms, structure and function}

Syntaxini, initially named p35, was first described by biochemical studies as 'two 35 -kilodalton proteins' (later known as syntaxin $\mathrm{Ia}$ and $\mathrm{Ib}$, respectively) that interact with the synaptic vesicle protein synaptotagmin and N-type calcium channels (Bennett et al., I992; Bennett et al., I993). The mammalian syntaxin family consists of I $_{5}$ different genes on different chromosomes. All of these syntaxins are type II oriented proteins, with amino terminus, the bulk of the polypeptide facing the cytoplasmic side. Most of the syntaxins are distributed ubiquitously while the predominant isoforms syntaxin Ia and $\mathrm{Ib}$ together with the less abundant syntaxin Ic, an alternative splicing product of syntaxin Ia (Jagadish et al., I997), are selectively distributed in neuronal and secretory cells (Teng et al., 200I). The main secretory cell specific isoforms, syntaxin Ia and syntaxin ib share more than $75 \%$ of homology in protein level, as well as the secondary structure, thus, probably also tertiary structure (Perez-Branguli et al., 2002). Even though there is no direct evidence to prove the functional differences between isoforms, wide range of literature suggest possible functional differences: in the light of their differential distribution in both central and peripheral nervous system (Aguado et al., I999; Ruiz-Montasell et al., I996), different Munci8 binding affinities (Perez-Branguli et al., 2002) and isoformpreferential co-localization with synaptobrevin (Perez-Branguli et al., I999). As Perez-Branguli et al. have shown, syntaxin Ia is preferentially localized with synaptobrevin/VAMP 2, and syntaxin Ib with synaptobrevin/ VAMP I, meaning 
that syntaxin Ia is predominant in the sensory neuron, while syntaxin Ib is exclusively expressed in motor neurons, suggesting that syntaxin $\mathrm{Ib}$ would be involved in neuronal networks, which need a very concrete amount of neurotransmitter release in a short time; conversely, syntaxin Ia could be related to a more regulated but slower exocytosis. The study on pancreatic beta cells revealed functional difference as well. In this system, they observed negative regulatory role in insulin release with syntaxin Ia overexpression, whereas insulin release did not show any difference in case of its isoform, syntaxin ib (Nagamatsu et al., I996). Biochemical approach on the long-term potentiation in mossy fiber terminals has shown increased expression of syntaxin Ib, together with increased capacity for glutamate release (HelmeGuizon et al., I998). All of these independent studies on the difference of isoforms correlatively allow the hypothesis that synapses with syntaxin ra could be more finely regulated, while those with $\mathrm{StxIB}$ would be bigger and/or more immediate responding.

Findings on differential regulation of N-type $\mathrm{Ca}^{2+}$ channel between isoforms also support this hypothesis. Coupling of exocytosis with excitation of the presynaptic terminals in brain is dominated by $\mathrm{Ca}^{2+}$ influx through $\mathrm{N}$ - and P/Q-type $\mathrm{Ca}^{2+}$ channels (Takahashi and Momiyama I993; Wheeler et al., I994; Bezprozvanny et al., 2000). Syntaxin binds to these $\mathrm{Ca}^{2+}$ channels (Bennett et al., I992) maybe to anchor synaptic vesicles near the $\mathrm{Ca}^{2+}$ entry site. Furthermore, syntaxin I has been reported to play a role as a negative regulator of the channels. Syntaxin I co- 
expressed with $\mathrm{N}$ - or $\mathrm{P} / \mathrm{Q}$-type $\mathrm{Ca}^{2+}$ channel in Xenopus oocyte, reduced the availability of the channel by stabilizing inactivated channel (Bezprozvanny et al., 1995; Wiser et al., 1996).

Another way of $\mathrm{Ca}^{2+}$ regulation by syntaxin I is a G-protein mediated manner (Stanley and Goping, 199I; Stanley and Mirotznik, 1997). They showed that expression of syntaxin Ia causes G-protein mediated $\mathrm{Ca}^{2+}$ current inhibition in calyxtype nerve terminal of the chick ciliary ganglion synapses, which can be reversed by a preceding depolarization or cleavage of syntaxin I with botulinum toxin CI (BTCI) (Stanley and Mirotznik, 1997). Parallel results were observed in tsA-20I cells. They found negative shift in the steady-state inactivation curve as well as G-protein mediated tonic inhibition. Interestingly, this G-protein mediated $\mathrm{Ca}^{2+}$ channel inhibition is caused only by syntaxin Ia, but not syntaxin Ib (Jarvis et al., 2000) (Jarvis et al., 2002). Moreover, when syntaxin I was co-expressed with either SNAP25 or Munc-18 additionally, the negative shift of the steady state inactivation caused by syntaxin I disappeared, whereas G-protein mediated tonic inhibition still persists (Jarvis and Zamponi, 200I). Thus, in the physiological condition, where all the interacting molecules of syntaxin I are present concomitantly, G-protein mediated regulation by syntaxin ra could be more important than direct inhibition.

If this isoform-specific regulation of $\mathrm{Ca}^{2+}$ channels causes difference in net $\mathrm{Ca}^{2+}$ influx at the presynaptic terminal, it would correlate well with the hypothesis 
that these two isoforms could bring different efficiency of membrane fusion. To address this question, we studied synapses with only syntaxin ib as compared to synapses containing both isoforms of syntaxin I, with respect to the parameters determining neurotransmitter release.

As described in part 2.6, release of syntaxin I into an open-conformation is required to allow syntaxin I to associate into the SNARE complex (Munson and Hughson, 2002; Weimer et al., 2003b). Since SNAP-25 or synaptobrevin does not have a self-regulatory function, switching conformation of syntaxin I is the core determinant of SNARE complex formation. However, in spite of its importance, the functional consequence of syntaxin I conformation change from closed to open state is largely not known.

Free syntaxin I exists in a dynamic equilibrium state between its open and closed conformations (Margittai et al., 2003b). If this equilibrium can be artificially shifted by genetic manipulations so as to obtain a specific predominant conformation, then the functional significance of such a conformation switch upon SNARE-mediated membrane fusion. Double mutation in the linker domain (Li65A, Ei66A) of syntaxin I has been reported to eliminate the default, closed conformation and lock syntaxin in its open conformation, thereby shifting the equilibrium to the constitutively active state. Also, this mutation abolished its binding ability to Munci8-I (Dulubova et al., I999). Thus, the second aim of our 
study was to understand the physiological consequence of open conformation of syntaxin $\mathrm{I}$.

To provide a comprehensive and in depth understanding of functional role of open conformation of syntaxin I in vesicular docking and membrane fusion, we characterized the mice expressing of syntaxin I with constitutively open conformation (double point mutation, Li65A, Eı66A; Gerber and Südhof, unpublished).

As described, the release of syntaxin into its open conformation is believed to be the main role of the essential priming factor, Muncizs. To resolve whether switching conformation of syntaxin I is indeed the sole function of Muncizs in membrane fusion, we tested whether activation of Muncizs via the diacylglycerol (DAG) stimulation, causes any further enhancement of neurotransmitter release over and above syntaxin I open mutation synapses 
3. Experimental Procedure 


\section{EXPERIMENTAL PROCEDURE}

\section{I Cell Culture}

\section{I.I Microisland Hippocampal Culture As a Model Synapse}

Microisland neuronal culture provided excellent experimental model synapse in this study. This central synapse model system is ideal for studying the most important parameters underlying synaptic transmission. In fact, it has been well established that these autaptic neurons are functionally indistinguishable from those in vivo including pharmacological kinetics, permeation properties as well as spontaneous activity (Bekkers and Stevens, I99I; Clements et al., I992).

Moreover, this serves as a powerful tool for highly reliable quantitative estimation of synaptic transmission. Since all synapses originate from a single axon and connect to the same neuron post-synaptically, different synaptic release modes can be quantified, including spontaneous neurotransmitter release activity as well as responses induced by an action potential or hypertonic solutions. All these synaptic parameters are collected from the same cell, which is ideal for parameter crosscorrelation, and also helps to reduce variability of data. One of the primary advantages of using this model system is that unlike conventional approaches, where pairs of neurons and electrodes are required, the autaptic system enables the use of a single electrode (Bekkers and Stevens I99I). 
To obtain autaptic hippocampal neuronal culture, neurons were cultured on the feeder-layer of astroglial microislands. Culture dishes with microislands of astrocytes were prepared as follows. First, we coated glass cover slips with coating material microdots. Entire sterile round glass cover slips (30 $\mathrm{mm}$ in diameter) were first coated uniformly with o.I5\% agarose (Type II-A, Sigma, Steinheim, Germany), a substrate upon which cells fails to attach, and placed in 6-well tissue culture plates. Coating material (o.I mM acetic acid, $0.2 \mathrm{mg} / \mathrm{ml}$ collagen, and o.I $\mathrm{mg} / \mathrm{ml}$ poly-Dlysine) is applied on top of the agarose using a rubber stamp with protruding pins to form small "islands", namely microdots, on which cells readily attach. By this method, we could provide distinct microisland area where cells can survive.

Next, astrocytes were enriched and plated using the following procedures. Brain cortexes of wild-type mice were enzymatically digested with a papain solution (20 units/ml of papain, $0.2 \mathrm{mg} / \mathrm{ml}$ cystein, I $\mathrm{mM} \mathrm{CaCl}{ }_{2}$ and $0.5 \mathrm{mM}$ EDTA in Dulbecco's modified Eagle’s medium (DMEM; GibcoBRL, Grand island, NY, USA) and bubbled with carbogen gas until the papain dissolved in completely) for an hour, followed by Io min-incubation in papain-inactivation solution $(2.5 \mathrm{mg} / \mathrm{ml}$ albumin, $2.5 \mathrm{mg} / \mathrm{ml}$ trypsin-inhibitor, and I0\% fetal bovine serum in DMEM). Subsequently, the brain tissue was gently triturated so as to obtain a single cell suspension. Cortical cells thus obtained were seeded and grown on $\phi 75-\mathrm{mm}$ tissue culture flask with Io\% FBS-DMEM until cells became confluent. Once confluent, the culture was vigorously vortexed for Io min and buoyant cells, which are mainly comprised of microglia, were discarded to enrich the astroglial population. 
Adherent cells were collected with trypsin-EDTA and plated on the prepared coated dishes with IO\% FBS-DMEM media containing IOO unit/ml of penicillin and IO० $\mu \mathrm{g} / \mathrm{ml}$ streptomycin (GibcoBRL, Grand Island, NY, USA). Approximately IO०,००० astrocytes were plated in each well of 6-well tissue culture plates. In the substrate forming microdot, astroglial as well as neuronal processes grow within the borders of the coated island but cannot reach outside because of the agarose. When astrocytes formed confluent islands, the anti-mitotic drug, FUDR (0.04 mM 2'deoxy-5-fluorouridine; I.O mM uridine) was applied for 24 hours to prevent astrocytes from overgrowing.
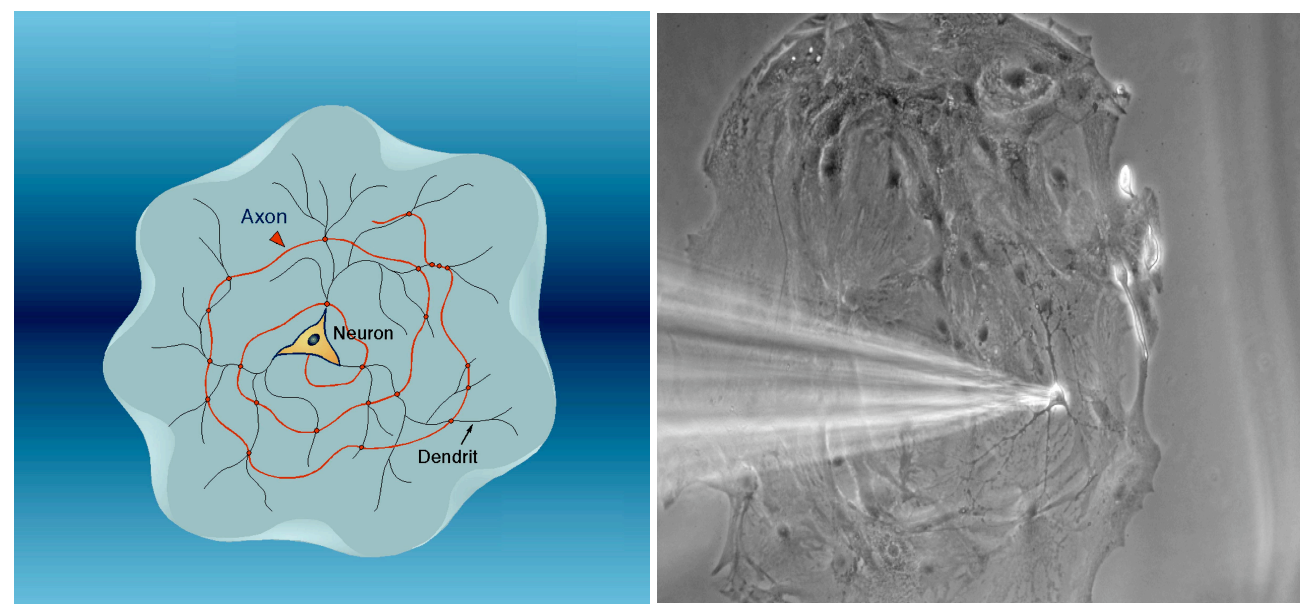

[Figure 4] Schematic diagram (left) and example (right) of autaptic neuron. On top of astroglial island, single neuron was grown to form all of synapses with its own dendrites. This model synaptic system is ideally suited to determine quantitatively the most important parameters underlying synaptic transmission. It is also unique, as all synapses originate from a single axon, which enables quantify different synaptic release modes including spontaneous release an responses by an action potential, by hypertonic solution in this study. 


\section{I.2Hippocampal Neuron Preparation}

Hippocampi were dissected out of neonatal Po mice brain in ice cold Hanks balanced salt solution (HBSS; GibcoBRL, Grand Island, NY, USA) and incubated with papain solution for $\mathrm{I}$ hr at $37^{\circ} \mathrm{C}$ with gentle shaking, followed by incubation with papain-inactivation solution for Io min. After removal of inhibition solution, the tissue was incubated in neurobasal A medium, with $2 \% \mathrm{~B}_{2} 7$ supplement, I\% glutamax I, $20 \mathrm{IU} / \mathrm{ml}$ penicillin and $0.02 \mathrm{mg} / \mathrm{ml}$ streptomycin (NBA medium) at $37^{\circ} \mathrm{C}$ with shaking at $900 \mathrm{rpm}$ while genotyping PCR reaction was completed. NBA medium and all of its supplements were purchased from GibcoBRL,Grand Island, NY, USA. Once we knew genotypes of littermates, hippocampi of homozygous mice were mechanically dissociated by gentle trituration in NBA medium. The tubes were allowed to stand for about 2 minutes so as to let the untriturated tissue sink-down by gravity. Dissociated single-cells in supernatant were transferred to fresh tubes, and cell number was determined. Approximately 4,000 to 5, 000 single neurons were plated on the prepared confluent microisland astrocyte culture. Cultures were incubated in NBA medium at $37^{\circ} \mathrm{C}$, with $5^{\circ}$ carbon dioxide and $95 \%$ humidity for 9 days or longer before being used for electrophysiological recordings. To reduce possible variation between culture plates, the two different groups of neurons being compared were seeded on the same plate. 


\subsection{Genotyping}

To preserve a homogeneous genetic background, we crossed heterozygote mice. In addition, comparing with control littermates, we were able to reduce the possible variability between different experimental animals. However, that required reliable genotyping of littermates. In order to genotype littermates, genomic DNA was enriched from the tail or fore brain of each animal. Primers for polymerase chain reaction (PCR) were designed to generate PCR products of different length, so that the wild-type and mutant gene were distinguished (S.Gerber and T.Südhof, unpublished).

\subsection{Genomic DNA Purification}

The template DNA was obtained from the tail biopsy from adult animals, or from brain tissue in case of neonatal mice and extracted using phenol/chloroform extraction. Tissues were incubated at $55^{\circ} \mathrm{C}$, with $300 \mu$ SNET buffers $(20 \mathrm{mM}$ Tris-HCl (pH 8.0), 5 mM Na_EDTA (pH 8.o), $400 \mathrm{mM} \mathrm{NaCl}$ and $\mathrm{I} \%$ sodium dodecyl sulfate (SDS)) for lysis of cells, along with $0.17 \mathrm{mg} / \mathrm{ml}$ Proteinase K (Roche Diagnostics, Mannheim, Germany) which breaks down polypeptides for better dissolution in phenol. Cell lysates were mixed with the same volume of a I:I mixture of phenol and chloroform (USB chemicals, Cleveland, the USA). These organic 
solvents precipitate proteins but leave nucleic acid in the aqueous phase. After $5_{5}$ min of centrifugation at $13,000 \mathrm{rpm}$, protein molecules are left as a white coagulated mass at the interface between the aqueous and organic phases. The upper aqueous phase containing nucleic acids was transferred into a clean tube.

The DNA in the aqueous phase was precipitated with 0.6 volume of isopropanol and pellets were washed twice with $70 \%$ ethanol, and then, dried out in a speed vacuum drier (Eppendorf, Hamburg, Germany). Purified DNA was resuspended in $300 \mu \mathrm{l}$ Tris-EDTA ( $\mathrm{o}$ mM Tris-HCl, I mM Na 2 EDTA; TE) buffer (pH 8.o) before PCR was performed.

Although the DNA extraction protocol described above (phenol-chloroform extraction) leads to relatively pure and high yield of DNA, it is not suitable to genotype during the preparation of neurons for the cell culture since it requires relatively long time, thereby subjecting the neurons to a suboptimal condition. Thus, phenol-chloroform extraction was used only for genotyping adult mice or confirming the results of genotyping during primary culture. Instead, quicker protocol (on-line genotyping) was used in the case of neonates for the purpose of culturing. Triturated brain was used because of the faster digestibility in this case. The brain tissues left-over after dissecting out the hippocampus from newborn pups were triturated and incubated in $0.5 \mathrm{ml}$ detergent free Tris-Sodium chloride buffer (Io $\mathrm{mM}$ Tris- $\mathrm{HCl}$ and $\mathrm{IOO} \mathrm{mM} \mathrm{NaCl}$; TS) with $0.17 \mathrm{mg} / \mathrm{ml}$ at $55^{\circ} \mathrm{C}$ with vigorous 
shaking for Io min. Undigested tissue was spun-down by centrifugation for $2 \mathrm{~min}$, and the proteinase $\mathrm{K}$ was inactivated by 5 minute boiling in a heating block. Another centrifugation of 3 minutes at $\mathrm{I} 3,000 \mathrm{rpm}$ was done to spin-down undigested tissue and coagulated matter. I-I.2 $\mu$ l of the supernatant was used as a template for the PCR.

\subsubsection{Genotyping PCR}

Two separate polymerase chain reactions (PCRs) were designed for detecting the type of gene present (S.Gerber and T.Südhof, unpublished). The reactions were performed according to [table 3] using a T-gradient ${ }^{\circledR}$ thermal cycler (Biometra, Goettingen, Germany). The amplified fragments were isolated by means of electrophoresis at $\mathrm{I} 2 \mathrm{O}-200 \mathrm{~V}$ in a $\mathrm{I} .8 \%$ agarose gel in Tris-Borate-EDTA (TBE: Ioo mM Tris-Cl (pH 8.o), I mM Na.EDTA, 90 $\mathrm{mM}$ borate) buffer. Ethidiumbromide (EtBr) was added in the gel and the PCR products were visualized under UV light. All chemicals used for gel electrophoresis were purchased from Life Technologies (Carlsbad, CA, USA), Gibco BRL (Grand Island, NY, USA), Roche (Indianapolis, IN, USA), Invitrogen (Grand Island, NY, USA) or Sigma (Steinheim, Germany). 


\section{A Wild type reaction}

Sense (SG01459): 5' GAA CGT CAG CTT CAG GCC TTC GCC TGC ATG 3 Antisense (SG01460):5'CCA GCT AGG GAA TAA TTA GACT AG GCG 3'

\section{Syntaxin 1 A knock out reaction}

Sense (SGO0368): 5' CAT AGC CT G AAG AAC GAG ATC AGC AGC CTC 3' Antisense (SG00395): 5' CT G CCT TTC CTG TGC CCT TAG GGG AAA GCC 3'

B

\begin{tabular}{|c|c|c|c|c|c|}
\hline \multicolumn{3}{|c|}{ wilc-type reaction } & \multicolumn{3}{|c|}{ knock-out reaction } \\
\hline Temp $\left({ }^{\circ} \mathrm{C}\right)$ & Time (m:s & & Temp $\left({ }^{\circ} \mathrm{C}\right)$ & Time (m:s) & \\
\hline 95 & 3:00 & & 95 & 3:00 & \\
\hline 95 & $0: 50$ & & 95 & 0:30 & \\
\hline 58 & $1: 10$ & 40 cycles & 66 & $1: 10$ & 40 cycles \\
\hline 72 & $2: 45$ & & 72 & $1: 00$ & \\
\hline 72 & $5: 00$ & & 72 & $3: 00$ & \\
\hline
\end{tabular}

\section{Wild type/stx1 Bof knock in reaction}

Sense: 5' CAA GGA CCG AAT CCA GAG GCA G $3^{\prime}$ Antisense :5' GCT CAC ATC ATC AGT GAA GAT GGC 3'

D

wilcttype reaction

Temp (으) Time (m:s)

\begin{tabular}{lll}
\hline 94 & $3: 00$ & \\
95 & $0: 30$ & \\
58 & $0: 45$ & 40 cycles \\
72 & $0: 45$ & \\
72 & $2: 00$ &
\end{tabular}

\section{E PCR-Mix}

per $25 \mu$ I Reaction

$$
\begin{aligned}
& 2.5 \mu \mathrm{l} 10 \times \text { Soriano Buffer } \\
& 0.3 \mu \mathrm{INTPS}(25 \mathrm{mM}) \\
& 2.5 \mu \mathrm{I} \text { Primer } 1(10 \mathrm{pmol} / \mu \mathrm{l}) \\
& 2.5 \mu \mathrm{I} \text { Primer } 2(10 \mathrm{pmol} / \mu \mathrm{l}) \\
& 0.2 \mu \mathrm{I} \text { Promega Taq }(5 \mathrm{unit} / \mu \mathrm{l}) \\
& 9.3 \mu \mathrm{l} \mathrm{H}_{2} 0 \\
& 1.0 \mu \mathrm{INA} \text { Demplate }
\end{aligned}
$$

\section{0x Soriano-Buffer}

166 mMAmmoniumsulphate $670 \mathrm{mMTris}-\mathrm{HCl} \mathrm{pH} 8.8$

$67 \mathrm{mM} \mathrm{MgCl}$.

50 mM B-Mercaptoethanol

$67 \mu \mathrm{MEDTA}$

in $\mathrm{H}_{2} \mathrm{O}$

[Table 2] Polymerase chain reaction for genotyping. For genotyping of Stx $1 \mathrm{~A} K O$ and Stx1Bof mice line, primers were designed as shown in $A$ and $C$ to generate different product in size. The reaction was performed in the condition described in $B$ and $\mathrm{D}$. The composition of PCR mixture was described in $\mathrm{E}$. 


\subsection{Protein assays}

\subsection{Immunoblot Analysis}

Immunoblot analysis allows quantitative estimation on the expression of a protein of interest. In the present study, this method was used to evaluate the amount of each isoform of Stxi. Extracted hippocampi were homogenized in lysis buffer (I50 mM NaCl, 5 mM EDTA (pH 8), I\% Triton X-ıоo, IO mM Tris-Cl (pH 7.4)), containing protease inhibitors ( $0.2 \mathrm{mM} \mathrm{PMSF}, 2 \mu \mathrm{g} / \mathrm{ml}$ each of aprotinin, pepstatin and leupeptin). Total protein concentrations of mice hippocampal lysates were determined by Bradford method (BioRad, Hercules, CA) using bovine serum albumin (BSA) as a standard. $20 \mu \mathrm{g}$ of total protein was boiled in sample buffer $(25 \%$ glycerol, 2\% SDS, I4.4 mM 2-mercaptoethanol, $3 \mathrm{mg} / \mathrm{ml}$ bromophenyl blue, and 60 mM Tris-HCl (pH6.8)) for Io min to make proteins have uniformed conformation and charge, eventually to resolve proteins only according to their molecular weight. Denatured proteins were resolved by sodium dodecyl sulfate - polyacrylamide gel electrophoresis (SDS-PAGE) and electrically transferred onto nitrocellulose membranes together with prestained molecular markers (Kaleidascope marer, BioRad, Hercules, CA, USA). To confirm proper transfer of proteins, the membrane was reversibly stained with Ponceau S (Sigma, Steinheim, Germany). The blotted membrane was incubated with blocking solution ( $5 \%$ skim milk, 5\% goat serum, 
$0.05 \%$ Tween 20 in Tris-buffered saline) for one hour at room temperature for blocking the membrane from non-specific protein binding and incubated either with monoclonal anti-StxiA antibody (Cl. 78.I) or polyclonal StxiB antibody, diluted I:IOoo in blocking solution. These antibodies were kindly provided by R. Jahn, MPI for biophysical chemistry and Synaptic System, Göttingen, Germany. The membranes were incubated with primary antibodies overnight at $4^{\circ} \mathrm{C}$ and then washed three times in TBS-T (0.05\% Tween20 in Tris-buffered saline). Subsequently they were incubated with horseradish peroxidase-conjugated secondary antibody for an hour at room temperature (I:IO,০oO; Jackson Immunoresearch laboratories, Pennsylvania, USA). After second wash in TBS-T, chemiluminescent detection was performed using ECL plus (Amersham, Uppsala, Sweden) and membranes were exposed to X-ray film (Amersham, Uppsala, Sweden).

\subsubsection{Immunohistochemistry}

We investigated a possible change in tissue distribution of syntaxin Ia and structural alteration of hippocampus caused by the lack of syntaxin $\mathrm{Ib}$ using immunohistochemistry. To reduce developmental differences, age-matched mice were used. Anaesthetized mice were intercardially perfused with 5-IO $\mathrm{ml}$ of ice cold heparinized saline, immediately followed by $50 \mathrm{ml}$ of fixative solution $(4 \%$ paraformaldehyde in o.I M sodium phosphate buffer). Then, the whole brain of each 
experimental group was extracted, and incubated with fixative solution for $30 \mathrm{~min}$ before being frozen. $14 \mu \mathrm{m}$ thick slices from the fixed and frozen brains were incubated with monoclonal anti-syntaxin Ia and polyclonal anti-syntaxin $\mathrm{Ib}$ antibodies (Synaptic system, Göttingen, Germany) and probed with Alexa Fluor 488 dye-conjugated anti-mouse antibody and Alexa Fluor 568 dye-conjugated anti-rabbit antibody. Primary and secondary antibodies were applied with $0.3 \%$ Triton X-Ioo to increase cellular penetration. As negative controls, wild-type brain slices were sustained using the same procedure with no primary antibody incubation. The processed slides prepared were mounted with mounting solution (I.5\% N-propyl gallate, 60\% glycerol in PBS) and analyzed by Confocal microscopy.

\subsection{Electrophysiology}

Electrophysiological recordings were done using the hippocampal autaptic neuron model system (figure 4: see part 3.I, Cell culture in this section). Around the same number of cells from each experimental group were recorded in a day to even out possible changes in synaptic release properties during development.

\subsection{Experimental Condition}

All electrophysiological recordings were carried out at room temperature $\left(23-25^{\circ} \mathrm{C}\right)$. Cultured autaptic neurons were perfused with the standard recording solution at a flow of $\mathrm{I} \mathrm{ml} / \mathrm{min}$ approximately. The solution in the dish was kept at a 
constant volume by a low-pressure aspiration system. Pharmacological agents and other solutions were applied using a fast flow application system, which allowed reliably rapid exchange of solutions in as little as $20-50 \mathrm{~ms}$. The patch pipettes were prepared using a horizontal puller (model p-87, Sutter of instrument, Novato, USA) from glass capillaries (type GBI5oF-8P, Science Products, Hofheim, Germany). Pipettes were pulled to a tip opening corresponding to about 2.2-3 $\mathrm{M} \Omega$ in chloridebased internal solution.

\subsubsection{Data acquisition}

Currents were filtered at $5 \mathrm{kHz}$ and recorded using a patch-clamp amplifier (Axopatch 200A; Axon Instruments, Union city, CA, USA), and were digitalized at Io kHz using Digidata I32IA and Clampex 8.03 software (Axon Instruments, Union city, CA, USA) and subsequently analyzed using Axograph 4.5 software (Axon Instruments, Union city, CA, USA).

\subsubsection{Standard external and internal solutions for electrophysiology}

The standard external solution consists of I $40 \mathrm{mM} \mathrm{NaCl}, 2.4 \mathrm{mM} \mathrm{KCl,} \mathrm{IO}$ mM HEPES, IO mM glucose, $4 \mathrm{mM} \mathrm{CaCl}_{2}, 4 \mathrm{mM} \mathrm{MgCl}$. Osmolarity and $\mathrm{pH}$ were adjusted to about 300 mOsm and 7.4 respectively to maintain physiological conditions during recording. The internal solution was made as following: $12 \mathrm{mM}$ phosphocreatin, $0.3 \mathrm{mM}$ GTP-Na, $4 \mathrm{mM}$ ATP-Mg, 50 units/ml creatinphosphokinase, $0.6 \mathrm{mM} \mathrm{MgCl}_{2}$, I mM potassium-EGTA, I36 mM KCl, I7.8 mM 
HEPES, solution was adjusted to $\mathrm{pH} 7.4$ and $300 \mathrm{mOsm}$.

\subsubsection{Stimulation Protocols and Electrophysiological parameters}

\subsection{4-I Evoked response}

As the standard procedure, EPSCs were recorded every 5 s to monitor the quality of voltage clamping and evaluate non-specific changes in EPSC amplitude such as the influence of time-dependent run-down, leaky seals etc. Neurons were somatically voltage-clamped in the whole cell patch clamp configuration at a holding membrane potential of $-70 \mathrm{mV}$. The cells were depolarized to $\circ \mathrm{mV}$ for $2 \mathrm{~ms}$ using a 0.2 $\mathrm{Hz}$ stimulation protocol to evoke action potentials, leading to an immediate $\mathrm{Na}^{+}$ inward current followed by $\mathrm{K}^{+}$outward current (figure 5 left). As described in Introduction (part 2.I), depolarization of membrane and subsequent action potential propagation induces $\mathrm{Ca}^{2+}$ influx in the presynaptic terminal which triggers a cascade of events evoking neurotransmitter release which can be detected as a post synaptic inward transient of quantal events normally with a 2-3 ms synaptic delay (EPSC in case of excitatory cells; Figure 5). The height of the peak and integration of the postsynaptic response were measured for EPSC amplitude and charge respectively. 


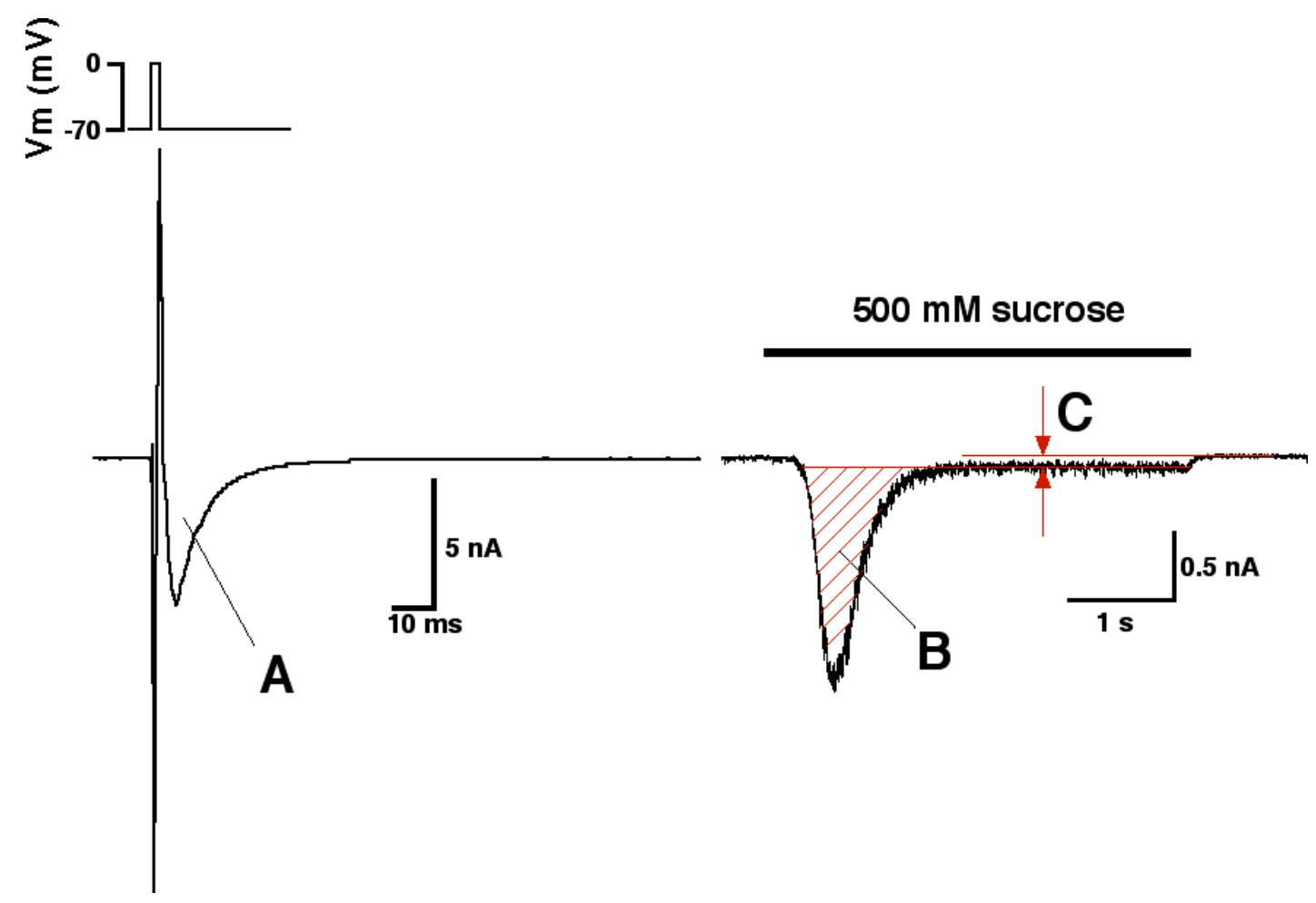

[Figure 5] EPSC, RRP and calculation of vesicular release probability. Left, Depolarization induced $\mathrm{Na}^{+}$inward and $\mathrm{K}^{+}$outward, and excitatory post synaptic currents (EPSC ;A). Right, Hypertonic solution (500 mM sucrose for 4 s)-evoked Inward transient current signifying the release of a readily releasable pool (RRP; B) of quanta (presumably corresponding to the primed synaptic vesicles) and followed by a steady state component representing equilibrium state between release and refilling. The mean amplitude of the steady state component quantifies refilling $\mathbf{C}$ (Rosenmund and Stevens, 1996). The charge of the EPSC (A in the figure) divided by the charge of RRP ( $B$ in the figure) gives the vesicular release probability. 


\subsection{4-2 Determining the size of readily releasable pool and vesicular release probability}

As a manner of determining the total charge of RRP, the charge induced by hypertonic solution was measured as described in (Rosenmund and Stevens, 1996). $500 \mathrm{mM}$ sucrose was extracellularly applied for more than $3 \mathrm{~s}$ using the fast flow application system. This application induces release of RRP, which leads to a transient followed by a steady state inward current (Figure 5, right). Since the transient part consists of a burst-like release of all fusion-competent, primed vesicles, the integral of the transient component after subtraction of steady state component give the total charge of the RRP (Figure 5 B). The sustained component induced by sucrose application is believed to represent the release of vesicles that have just been primed into the RRP (Figure 5 C; Reim et al., 200I).

To determine the vesicular release probability, $500 \mathrm{mM}$ sucrose were applied for $4 \mathrm{sec}, 3 \mathrm{sec}$ after evoking the action potential. The vesicular release probability was calculated as the charge released by an action potential divided by total charge of the RRP [Figure 5]. 


\subsection{4-3 Short-term plasticity}

Cells were stimulated at Io or $20 \mathrm{~Hz}$ frequencies so as to reveal the shortterm plasticity characteristics. Peak amplitudes of EPSCs during each train were measured and averaged. Furthermore, to reduce cell-to-cell variation in amplitude, every measured response was normalized to the amplitude of the first EPSC, and then averaged.

\subsection{4-4 Calcium Sensitivity of evoked neurotransmitter release}

The efficiency of the $\mathrm{Ca}^{2+}$-triggered neurotransmitter release is one of the most important factors that decide the vesicular neurotransmitter release probability. Thus, to evaluate possible changes in the relationship between EPSCs and the external $\mathrm{Ca}^{2+}$ concentration by a given mutation, we studied neurotransmitter release as a function of external $\mathrm{Ca}^{2+}$ concentration.

The sensitivity of synaptic responses to various concentrations of external $\mathrm{Ca}^{2+}$ was measured at constant $\mathrm{Mg}^{2+}$ concentrations (I $\mathrm{mM}$ ). To control for the rundown of synaptic responses, individual test measurements were preceded and followed by EPSC measurements under standard conditions, namely $4 \mathrm{mM}\left[\mathrm{Ca}^{2+}\right]_{\mathrm{ex}}$ and $4 \mathrm{mM}\left[\mathrm{Mg}^{2+}\right]_{\mathrm{ex}}$. EPSCs in various $\left[\mathrm{Ca}^{2+}\right]_{\mathrm{ex}}$ are taken after being stabilized, and compared to mean EPSC amplitudes of preceding and following standard conditions. As an example, in figure 6, the level of EPSC change was determined as $2 \mathrm{~B} /(\mathrm{A}+\mathrm{C})$. A represents the averaged EPSC amplitude of a standard condition $(4$ 
$\mathrm{mM} \mathrm{Ca}^{2+}$ and $\left.4 \mathrm{mM} \mathrm{Mg}{ }^{2+}\right)$ before varying $\left[\mathrm{Ca}^{2+}\right]_{\mathrm{ex}}$; shows mean EPSC amplitude under experimental condition ( $12 \mathrm{mM} \mathrm{Ca}^{2+}$ and I $\mathrm{mM} \mathrm{Mg}^{2+}$ in figure 6) and $\mathrm{C}$ signifying average EPSC in standard condition after changing $\left[\mathrm{Ca}^{2+}\right]_{\mathrm{ex}}$.

The change in $\mathrm{Ca}^{2+}$-sensitivity of neurotransmitter release will lead to alterations in the level of potentiation at high $\left[\mathrm{Ca}^{2+}\right]_{\mathrm{ex}}$ and also reduction of EPSC at low $\left[\mathrm{Ca}^{2+}\right]_{\mathrm{ex}}$.

On the other hand, in case $\mathrm{Ca}^{2+}$-independent vesicular release probability is changed (even without affecting $\mathrm{Ca}^{2+}$-sensitivity), level of potentiation can be expected to be altered because high probability of release limits the potential for any further enhancement while lower release probability allows a greater degree of potentiation. 


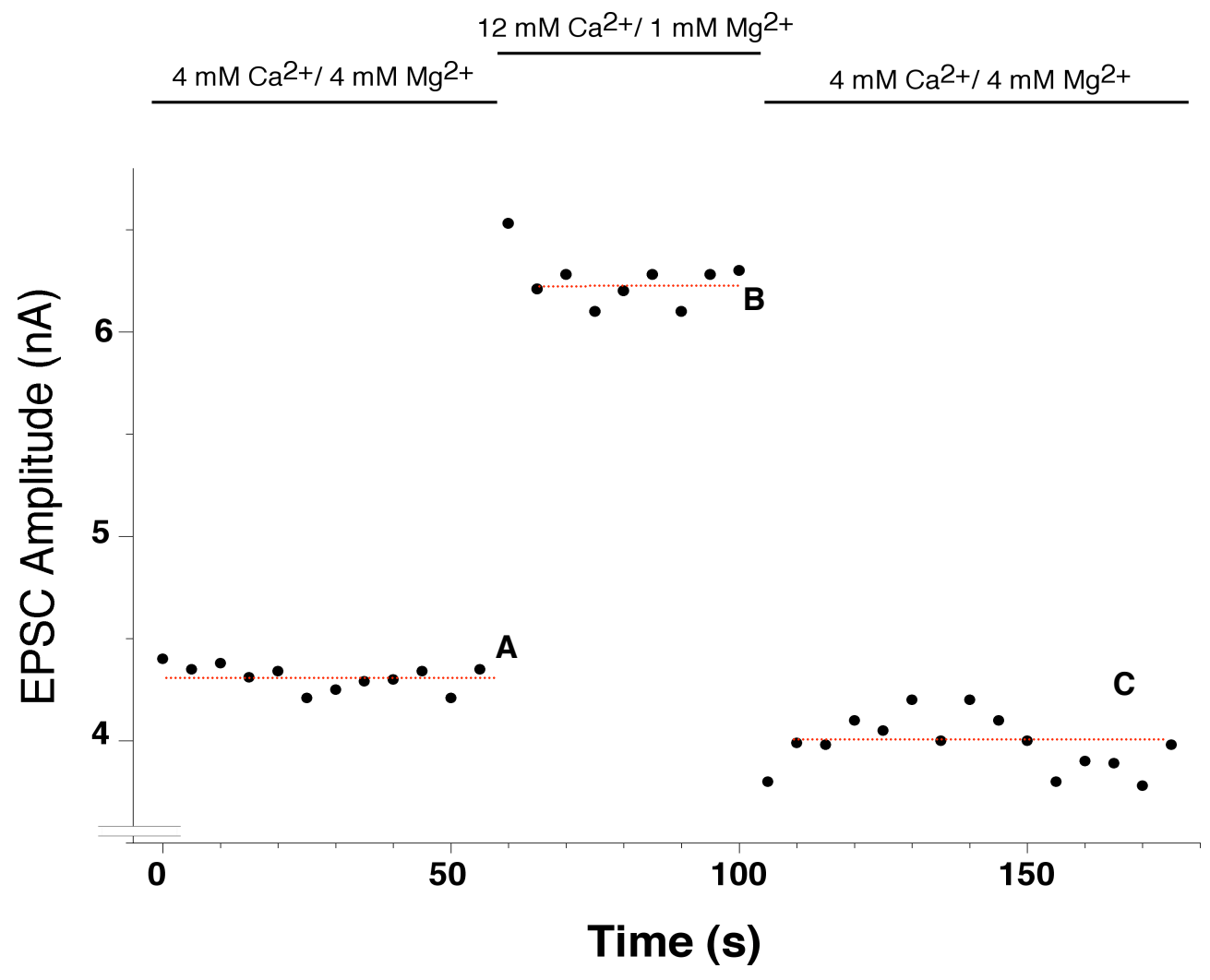

[Figure 6]Calculation of EPSC change in various $\mathrm{Ca}^{2+}$ concentrations. The sensitivity of synaptic responses to various $\left[\mathrm{Ca}^{2+}\right]_{\mathrm{ex}}(12 \mathrm{mM}$ in this figure) was measured at constant $\mathrm{Mg}^{2+}$ concentrations $(1 \mathrm{mM})$. To control the rundown of synaptic responses, individual test measurement was preceded and followed by EPSC measurements under standard control conditions, $0.2 \mathrm{~Hz}$, in $4 \mathrm{mM}\left[\mathrm{Ca}^{2+}\right]_{\mathrm{ex}}$ and $4 \mathrm{mM}\left[\mathrm{Mg}^{2+}\right]_{\mathrm{ex}}$. Dashed lines show averages of each measurement under standard (before (A) and after (C)) and experimental $(B)$ conditions. The level of augmentation was calculated as $2 \mathrm{~B} /(\mathbf{A}+\mathbf{C})$. 


\subsection{4-5 Synaptic release probability}

Synaptic release probability differs from vesicular release probability by the probability that any vesicle, no matter how many, at a given synapse is released during an action potential, note that vesicular release probability is the ratio of the charge released by an action potential to that of RRP. This can be different because of non-uniform size and structure of synaptic terminal. As a way of measuring synaptic release probability, the NMDA component of EPSC was measured with IOO - continuous action potentials in presence of MK-8OI, the irreversible NMDA open channel blocker. Since the antagonist will only be able to lock the pore of the receptor channel, only channels opened before are blocked. Thus, if the release probability of a given synapse is high, more post-synaptic NMDA channels will be open, and the progressive block should be faster (Rosenmund et al., I993).

NMDA-EPSCs were revealed in external solution containing $2.7 \mathrm{mM}$ external $\mathrm{Ca}^{2+}$ and Io-mM glycine, without any added $\mathrm{Mg}^{2+}$ (see glutamate receptors in introduction part 2.2.3). This modified external solution allowed us to measure both AMPA and NMDA component of EPSC.

The amplitude of the NMDA component was measured by Ioo successive stimuli at every 5 seconds in presence of $5 \mu \mathrm{M}$ MK-8oI. The rate of progressive block of NMDA-EPSCs was estimated to evaluate synaptic release probability over synapses of entire neuron (Rosenmund et al., I993). 
Since this protocol takes relatively long time, there could be significant rundown of synaptic response in addition to decaying by MK-80I. To compensate for the non-specific change of EPSC amplitude, every peak current of AMPA component of the individual response was set to one and normalized NMDA currents were measured by the mean amplitude between $50 \mathrm{~ms}$ and IOO ms (or later, depending on the size of the AMPA component).

\subsection{4-6 Spontaneous release}

Miniature EPSCs (mEPSCs or minis) were recorded at $-70 \mathrm{mV}$ at least for 5os with $0.3 \mu \mathrm{M}$ tetradotoxin (TTX) to completely block the possibility of undesired action-potentials. A template-based detection program within the function of Axograph 4.5 was used for detecting and analyzing the miniature events. The template of mEPSC was determined as the following equation and criteria:

$$
f(t)=\exp (-t / \text { rise time })-\exp (-t / \text { decay time })
$$

Template amplitude $=-20 \mathrm{pA}$, template rise time $=0.5 \mathrm{~ms}$, decay time $=5 \mathrm{~ms}$, base line before events $=5 \mathrm{~ms}$, and total template length $=30 \mathrm{~ms}$. When the amplitude or charge was evaluated, events spaced within Io ms of one another were discarded. Detection threshold was set to -3.5 fold of the noise standard deviation. Detected events were reselected based on their amplitude, rise time, and half-width. Events within the range of Io to IOO $\mathrm{pA}$ in amplitude, $O$ to $\mathrm{I} \mathrm{ms}$ in rise time, and $\circ$ to $5 \mathrm{~ms}$ in 
half-width were considered as mini events. Selected events of individual cell were averaged to determine the mean amplitude and charge.

The number of vesicles in the RRP was calculated by dividing the integrated charge of the transient burst component in the sucrose response by the mean mEPSC charge measured for each cell. By comparing the frequency of events to the number of vesicles in RRP, spontaneous release activity was normalized for variation in size of RRP, which defines the spontaneous release probability (pool unit/s).

\subsection{4-7 Somatic Calcium Current}

Somatic calcium currents were measured in the external solution containing O.5 $\mu \mathrm{M}$ tetradotoxin (TTX) which blocks the sodium current and Io $\mathrm{mM}$ tetraethyl ammonium (TEA) to obstruct potassium current and we used Cesium-gluconate based internal solution to block potassium conductance. Cells were held at $-70 \mathrm{mV}$ and depolarized to $\circ \mathrm{mV}$ for Io ms. Traces were subtracted by the current with external $200 \mu \mathrm{M}$ cadmium, which completely blocks all types of $\mathrm{Ca}^{2+}$ current without affecting the $\mathrm{Na}^{+}$or $\mathrm{K}^{+}$current, to rule out possible interferences of other ionic flow. 
Because mainly $\mathrm{P} / \mathrm{Q}$ - and $\mathrm{N}$-type $\mathrm{Ca}^{2+}$ channels govern the $\mathrm{Ca}^{2+}$ influx in the presynaptic terminal (see (Catterall, I999)), $3 \mu \mathrm{M}$ nifedipine was applied to eliminate the L-type channel dependent calcium current out of total $\mathrm{Ca}^{2+}$ current to provide better resolution of presynaptic $\mathrm{Ca}^{2+}$ current.

\section{$3 \cdot 4 \cdot 4-8$ Kinetics of sucrose responses}

To get a preliminary idea on neurotransmitter release, the release time course during sucrose application was analyzed. First, averaged shapes of sucrose responses were gained. Before averaging, each sucrose response was aligned at its peak of the transient component to determine width of the response. In another side, the evoking time delay was measured independently from individual sucrose response. Average shapes of sucrose responses were shifted according to the average time to evoke. To avoid artifacts resulting from improper application of the solution, comparable numbers of cells were analyzed on a given day and responses delayed by more than one second from the point of digital switching, were ruled out in this study. To compare release properties of each group, sucrose responses were integrated with a zero line as the sustained component and normalized by the total charge of the pool in each cell to standardize different RRP sizes.

Then, for more accurate analysis of hypertonic sucrose solution-evoked responses, the time-to-peak, half-width, I0-90\% rise time, and time-to-onset of individual transient components were analyzed using Axograph 4.5 (Axon instruments, Union city, CA, USA). Responses with values lying outside of three 
times standard deviation in any of the mentioned parameters were ruled out in this study.

\subsection{4-9 Osmotic pressure-dependent neurotransmitter release}

Neurons with greater release rates require less energy to have the same charge of transient component, since the transient component of a hypertonic solution-induced response represents the faster depletion factor in comparison to the sustained component. We therefore compared 250 and $35^{\circ} \mathrm{mM}$ sucrose-evoked transient component between the experimental groups. The charge was measured in the same way depicted in figure 5 and the released charge was normalized by the RRP charge of individual cells.

\subsection{4-IO Maximum release rate}

The maximum slope of the integrated sucrose response was determined in terms of maximum pool units released per second and used to compare the efficiency of neurotransmitter release by mechanical force. To compare neurotransmitter release rate as a function of various osmotic pressures, cells were subjected to IOO, $250,35 \circ, 500 \mathrm{mM}$ sucrose solutions. To calculate the maximum release rate by $250,35^{\circ}$ and $500 \mathrm{mM}$ sucrose, each transient component of sucrose response was integrated after baselining at the sustained component, and then normalized by the total charge of RRP judged by the released charge of the transient component during a $500 \mathrm{mM}$ sucrose application, to even out various 
RRP sizes. To measure the relese rate triggered by $100 \mathrm{mM}$ sucrose, we used miniature events detection program within the function of Axograph 4.5 as described in spontaneous release section and calculated release rate based on the frequency of mini events. Measured frequency in presence of Ioo $\mathrm{mM}$ sucrose was divided by the corresponding number of vesicles in RRP (calculated as charge of transient component caused by $500 \mathrm{mM}$ divided by mean charge of spontaneous release).

\subsection{4-II Rate of vesicle turn over or refilling of readily releasable pool}

The rate of replenishment of the RRP was compared by two different methods. The mean amplitude of the sustained component of a sucrose response was determined as the refilling amplitude, since the steady state sustained amount of release after depleting RRP is believed as the release from the vesicles just primed. The values were normalized to the total charge of the RRP of individual cell to compare refilling portion of the pool in a second (pool unit/s). However, this measurement is often not feasible, because of relatively low signal to noise ratio.

To overcome this problem, a paired pulse of sucrose application protocol was used. Two pulses of sucrose were applied onto the cell at variable time intervals. Then, the refilling rate during a time interval between two sucrose applications was determined by the ratio of the second release over the first release. 


\subsection{4-I2 PDBU-induced augmentation}

Augmentation of neurotransmitter release by $\beta$-phorbol ester $(\beta$-PE), a functional analogue of the endogeneous second messenger, diacyl glycerol (DAG) had been believed as a consequence of protein kinase $\mathrm{C}$ activation before it was found that no augmentation can be achieved by $\beta$-PE in neurons expressing mutant Munciz deficient in DAG binding (Rhee et al., 2002). Since it is now well accepted that the $\beta$-PE induced augmentation is mediated by Muncizs, the level of augmentation was measured to examine whether Munci3 plays an additional role besides switching the conformation of syntaxin to the open state.

After obtaining baseline EPSC in standard condition, I $\mu \mathrm{M} \beta$-PE was applied for I min, during $0.2 \mathrm{~Hz}$ stimulation, and then the effect of washing it out was measured for another $5 \mathrm{~min}$. All of measured EPSCs were normalized to initial baseline EPSC and then averaged. The degree of augmentation was determined from the augmented EPSC after it reached steady state.

To resolve whether decreased augmentation in $\mathrm{StxIB}_{\text {of }}$ is caused by increased initial release probability, the levels of augmentation caused by $\beta$-PE for both groups was plotted in a graph as a function of vesicular release probability for each cell. Exponential fits were gained using a curve fit function of Kaleidagraph 3.०.

\subsection{Experimenter bias minimization}


Having prepossession during experiment and analysis of data can make significant effect on the results. To rule out any artifact that the experimenter could make by prejudice, all electrophysiological recordings and analysis were performed without knowledge of genetic background of the culture to eliminate experimenter bias artifact. A. Meyer regularly analyzes the genotyping results and assigned the mice of the different genotypes as letters A, B, C etc.

\subsection{Data display and Statistics}

All data shown represent the mean \pm S.E.M. Statistical comparisons were performed using Mann-Whitney Test using statistical software Instat version 2.03 (GraphPad software, USA). A $p$ values below $\circ \cdot 05$ was regarded as a statistically significant difference. 
4. Results 


\section{RESULTS}

Being an integral component of the SNARE complex, syntaxin I plays a major role in neurotransmitter release via SNARE-mediated synaptic vesicle fusion. As described in the Introduction (part 2.9) syntaxin (Stx) has I5 different isoforms, and StxiA and StxiB are the two major isoforms expressed exclusively in secretory cells. Previous experiments showed differences between StxIA and StxiB including differences in distributions, co-localization with other SNARE partners, $\mathrm{Ca}^{2+}$ channel regulation, binding affinity to Munci8-I (Aguado et al., I999; Jarvis et al., 2000; Jarvis and Zamponi, 200I; Lu et al., 200I; Perez-Branguli et al., 2002; PerezBranguli et al., I999; Ruiz-Montasell et al., I996). Although these studies suggest the possibility of functional differences between StxiA and StxiB, it is not yet documented whether these isoforms indeed have distinct roles in neurotransmitter release.

Stxı is implicated to be the key determinant in regulating actual formation of SNARE complexes by its conformational switch (Duluvoba et al., I999, Margittai et al., 2003a, Richmond et al., 200I). However, the physiological outcome of openconformation of StxI remains unclear. Furthermore, in spite of the elegant study in C.elegans showing that opening of Stx is the sole function of Unci3 (Richmond et al., 200I), it has not yet been documented in mammalian system.

To address these questions, we characterized two genetically manipulated mouse lines, namely StxiA null mice (StxiAKO) and double mutant mouse 
expressing the constitutively open conformation of StxIB and lacking StxiA $\left(\mathrm{StxiB}_{\mathrm{of}}\right)$ using interdisciplinary methods including western blotting, immunohistochemical and electrophysiological techniques.

\section{I Mutant mouse strains, StxIAKO}

To examine the possible isoform-specific functional differences of Stxi by characterizing synapses exclusively expressing StxiB against those expressing both isoforms, we used StxiA null mice. Furthermore, it is necessary to identify the effects of eliminating StxiA to provide a proper control for the second part of the study, which explores the physiological consequences of open-form Stxi by studying the synaptic release properties of $\mathrm{Stxi}_{\mathrm{of}}$.

StxiAKO mice were generated and provided by S. Gerber and T.Südhof at UT Southwestern, Dallas, TX, U.S.A. The line was maintained as heterozygotes to obtain mutant and wild-type in any given litter to eliminate possible litter-to-litter differences.

Homozygous StxiAKO mice are viable and fertile with no detectable abnormality in behavior or in gross anatomy (S.Gerber and T.Südhof, unpublished).

\subsection{Expression of StxIA and StxIB in wild-type, heterozygote and knock out mice}


To confirm these mouse models, we had to verify that the deletion of the gene leads to complete abolishment of StxiA expression. Therefore, we used western blot analysis against StxiA to verify the absence of StxiA at the protein level. Also to evaluate possible changes of expression level of StxiB protein, we quantified expression of StxiB in the absence of StxiA protein. The same amount of hippocampal extract was separated on SDS-PAGE electrophoresis and blotted onto nitrocellulose membrane. Blots were probed with isoform specific antibodies.

Figure 7 left shows that expression of StxiA was notably reduced in heterozygote mice (HZ) and completely abolished in StxiAKO mice. However, the lack of StxiA apparently did not affect the expression of StxiB (Figure 7 right). We conclude that in StxiA KO mice, StxiA protein is completely eliminated whereas StxiB expression remains unchanged, and the total amount of Stxi protein is reduced.

Since StxIAKO mice did not show any notable deficits in survival, behavior or anatomy, this demonstrates that neither absence of StxiA nor reduction in total amount of Stxi critically affected normal development and survival of mice. 


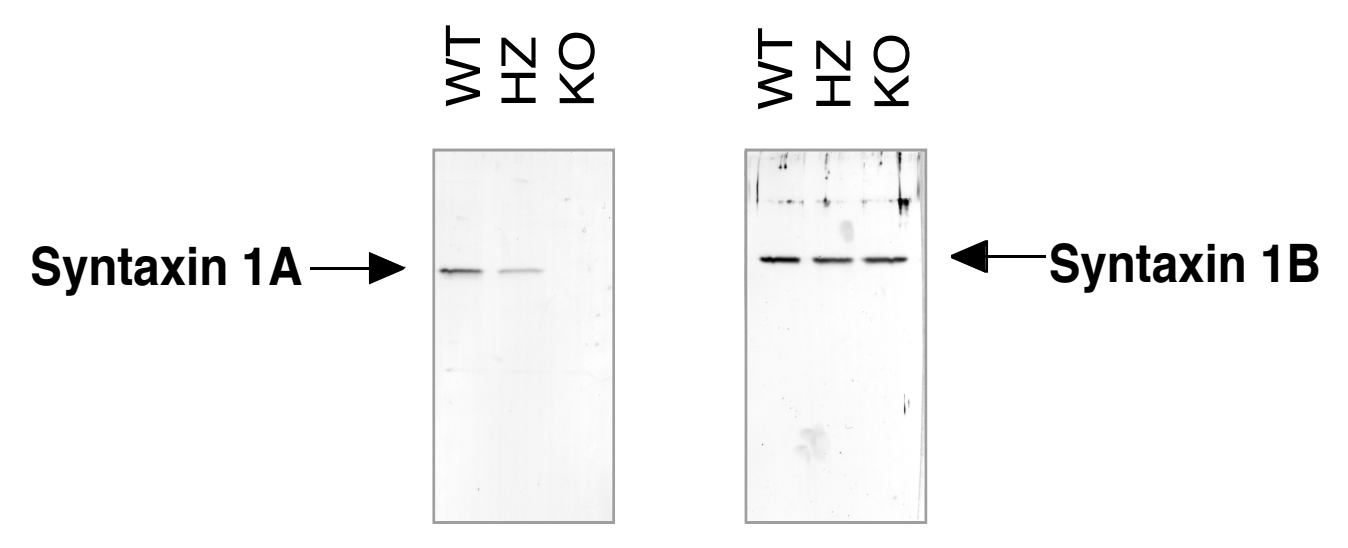

[Figure 7] Western blotting analysis Expressions of syntaxin 1a (left panel) and syntaxin $1 \mathrm{~b}$ (right panel) were analyzed using western blotting in wild type (WT), Stx1A heterogygotes (HZ) and Stx1A knock out (KO) mice. $20 \mu \mathrm{g}$ of total protein extracts were loaded in each lane, separated by SDS-PAGE and probed with monoclonal Stx1A (Cl. 78.1; left) or polyclonal Stx1B antibody (right). As expected the level of Stx1A expression was decreased in heterozygotes by half and knocked out in null mice, while expression of Stx $1 \mathrm{~B}$ is essentially constant. 


\subsection{Tissue distribution of StxIA and StxIB in hippocampus}

To verify the complete elimination of StxiA and to test out possible changes in brain structure or distribution of StxiB by deletion of StxiA protein, we performed immunohistochemical staining experiments on slices from adult hippocampus. Fixed and frozen brains of age-matched adult mice were sliced at I4 $\mu \mathrm{m}$ thick and probed with anti-StxiA and $\mathrm{IB}$ antibodies. As a negative control for non-specific staining, we stained wild-type brain slices using the same procedure omitting out the primary antibody incubation. Figure 8 a shows distribution of these two isoforms in wild-type brain, in contiguity with the previous report of RuizMontasell (Ruiz-Montasell et al., I996). The signal of StxiA was significant reduced in the brain of heterozygote mice and signal detected in StxiAKO brain was comparable to that of negative control, which confirms the complete absence of StxiA in StxiAKO brain. We observed no obvious structural changes neither in the HZ nor the StxiAKO brains $(n=2)$, implying that absence of StxiA does not significantly affect development of brain structure.

This result, along with that of western blot analysis consistently shows that genetic ablation of StxiA leads to complete absence of the protein and proves that our genotyping assay is reliable. Furthermore, we found that removal of StxiA does not alter the expression of StxiB gene. Therefore, we could conclude that StxiAKO mouse line serves an ideal experimental system to investigate isoform-specific 
function of Stxi. In addition, it allows direct comparison between synapses exclusively using wild-type StxiB versus those using StxiB with open-form mutation, in the second part of the study. 


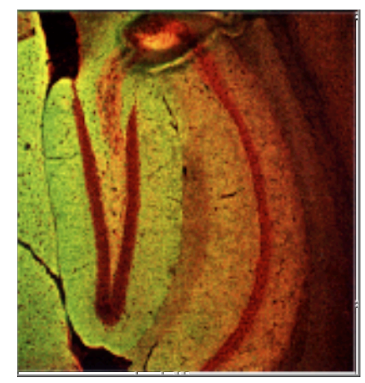

WT

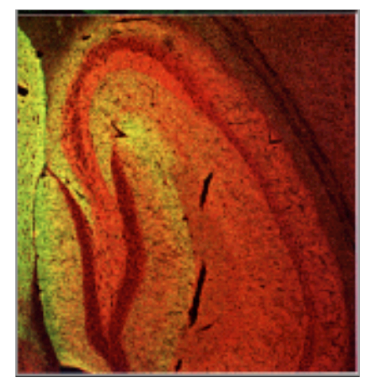

$\mathrm{HZ}$

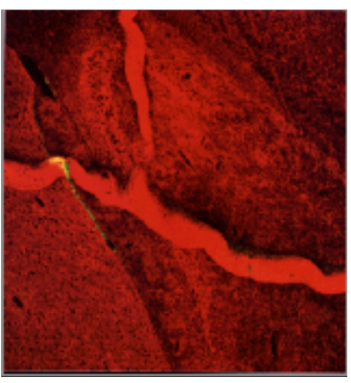

$\mathrm{KO}$

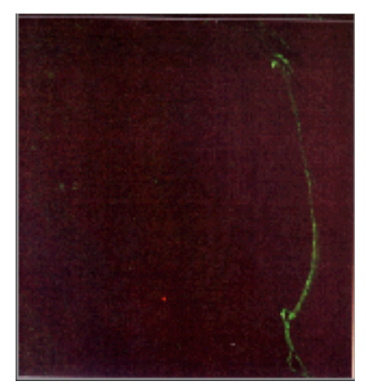

(-) control

[Figure 8] Distribution of Stx1A and Stx1B in hippocampus. Fixed and frozen whole brains were sliced in $14 \mu \mathrm{m}$-thick sections, and incubated with monoclonal Stx1A (Cl. 78.1), green; and polyclonal anti-Stx1B, red; followed by with secondary antibodies. Wild-type (WT), heterozygotes (HZ) and knock out (KO). Negative control is gained from the same protocol omitting primary antibody incubation. 


\subsection{Basic Characteristics in release efficacy of StxIA KO}

To study synaptic neurotransmitter release properties of synapses using only StxiB in comparison to those using StxiA and StxiB, we performed voltage-clamp recording on cultured individual hippocampal neurons from newborn pups (Bekkers and Stevens, I99I). We focused our analysis on excitatory glutamatergic neurons because they are more numerous, in addition, inhibitory GABAergic neurons show different molecular properties of vesicle release (Augustin et al., I999). GABAergic neurons can be easily distinguishable from glutamatergic neurons by their time course of evoked neurotransmitter release. Neurons were voltage clamped at $-70 \mathrm{mV}$ and synaptic responses were evoked by $2 \mathrm{~ms}$ of somatic depolarization.

Our comparison of the mean amplitudes of the EPSCs between StxiAKO mice and the control wild-type littermates, showed no obvious change in synaptic amplitude (figure 9A, 9B: $7.0 \pm 0.6 \mathrm{nA}, \mathrm{n}=76$ for $\mathrm{WT} ; 7.3 \mathrm{nA} \pm 0.6, \mathrm{n}=77$ for StxiAKO, $p=0.9253)$. This data indicate that complete removal of StxiA isoform lead to no significant impact on amplitude of evoked responses.

Next, we compared mean EPSC charge to detect possible changes in EPSC time course. If knocking out StxiA caused major alteration of EPSC time course, for an example increases in asynchronous component, charge of EPSC could be altered, while the peak amplitude remains constant. Also, the EPSC 
charge provided crucial information for calculating vesicular release probability. The measured mean EPSC charge was not affected upon removal of StxiA $(54 \pm 5$ pC, $\mathrm{n}=76$ for $\mathrm{WT}$ and $55.8 \pm 0.7 \mathrm{pC}, \mathrm{n}=77$ for StxIAKO, $\mathrm{p}=0.9824)$, supporting the conclusion that the lack of StxiA does not bring about considerable alteration of evoked synaptic neurotransmitter responses.

Another important factor that determines the efficiency of neurotransmitter release is that total number of vesicles available for fusion, readily releasable pool (RRP). RRP can be quantified in autaptic neurons by pulsed application of hypertonic solution.

Four-second application of the hypertonic solution causes a transient followed by the steady state inward current. The transient component subtracted by the sustained component represents RRP and the sustained component represents the release of vesicles, which newly become available (Rosenmund and Stevens, I996). We integrated the transient component of hypertonic sucrose response to define the RRP size and found no significant difference in their size between the StxiAKO and their W'T littermates (figure ${ }_{9} \mathrm{D}$ and ${ }_{9 \mathrm{E}}$ : $690 \pm 80 \mathrm{pC}, \mathrm{n}=68$ for $\mathrm{W} T$; $690 \pm 8 \circ \mathrm{pC}, \mathrm{n}=65$ for $\mathrm{KO}, p=0.9534)$

Knowing the charge of EPSCs as well as the charge of RRP, we can directly calculate which fraction of the RRP is released during the single action potential. This number is defined as vesicular release probability and it can be easily calculated by forming the ratio of the evoked response charge and RRP charge. We calculated vesicular release probability for each cell and found as expected that the vesicular 
release probability was unchanged in the StxIA deficient neurons $(0.104 \pm 0.007, \mathrm{n}=$ 68 for WT; O.IOI $\pm 0.006, \mathrm{n}=65$ for StxiAKO, $p=0.9193$, Figure $9 \mathrm{~F})$. This finding indicates that regardless whether StxiA is present or absent, an action potential releases synaptic vesicles with the chance of approximately IO\%.

\subsection{Short-term plasticity characteristics in StxIAKO neurons}

Presynaptic short-term plasticity is critically governed by changes in the vesicular release probability, along with other parameters such as action poteinalinduced $\mathrm{Ca}^{2+}$ influx and accumulation, RRP size, and pattern of stimulation (see (Zucker and Regehr, 2002)). Synapses with high probability of neurotransmitter release tend to depress during tetanic stimulation because limited number of fusioncompetent vesicles are more quickly consumed than replenished. This phenomenon is clearly elucidated in the case of synapses expressing Munci3-I $\mathrm{H}_{5} 67 \mathrm{~K}$, point mutant knock-in mice that render Munci3-I insensitive to diacylglycerol/ $\beta$-phorbol ester, which showed stronger depression of synaptic responses during trains of action potentials because of their initially higher release probability in comparison to wild-type littermates (Rhee et al., 2002). On the other hand, low release probability synapses like those lacking complexin I,2 or expressing mutant synaptotagmin ( $\mathrm{R} 233 \mathrm{Q})$, which have 2-fold apparent decrease in $\mathrm{Ca}^{2+}$ sensitivity of release and thus reduced vesicular release probability, tend to facilitate (Fernandez-Chacon et al., 200I; Reim et al., 200I), probably because of accumulated residual presynaptic $\mathrm{Ca}^{2+}$ 
(Schneggenburger et al., 2002). In contrast, wild-type hippocampal autaptic neurons consistently show approximately $50 \%$ of depression under identical condition.

Therefore, to further confirm the effect of StxiA removal protein on initial release probability and subsequently on synaptic depression, we analyzed the normalized synaptic responses of amplitude during 50 trains of action potentials at a frequency of IoHz.

We quantified peak current of each evoked response of the spike train and plotted the mean amplitude of all cells recorded (Figure $9 G$ ). In addition, we normalized EPSCs to amplitude of the first EPSC to reduce the cell-to-cell scatter in amplitude and then averaged (Figure $9 \mathrm{H}$ ). As shown in figure $9 \mathrm{G}$ and $9 \mathrm{H}$, both groups exhibited a typical short-term plasticity pattern of wilt-type neuron, prominent depression of EPSC during IO $\mathrm{Hz}$ trains, which reach equilibrium at $45 \%$ for WT and $48 \%$ for StxiAKO of the initial amplitude. Therefore, the patterns of short-term plasticity during Io $\mathrm{Hz}$ in hippocampal neuronal autapses with and without StxiA are essentially the same as expected by the finding concerning vesicular release probability. 

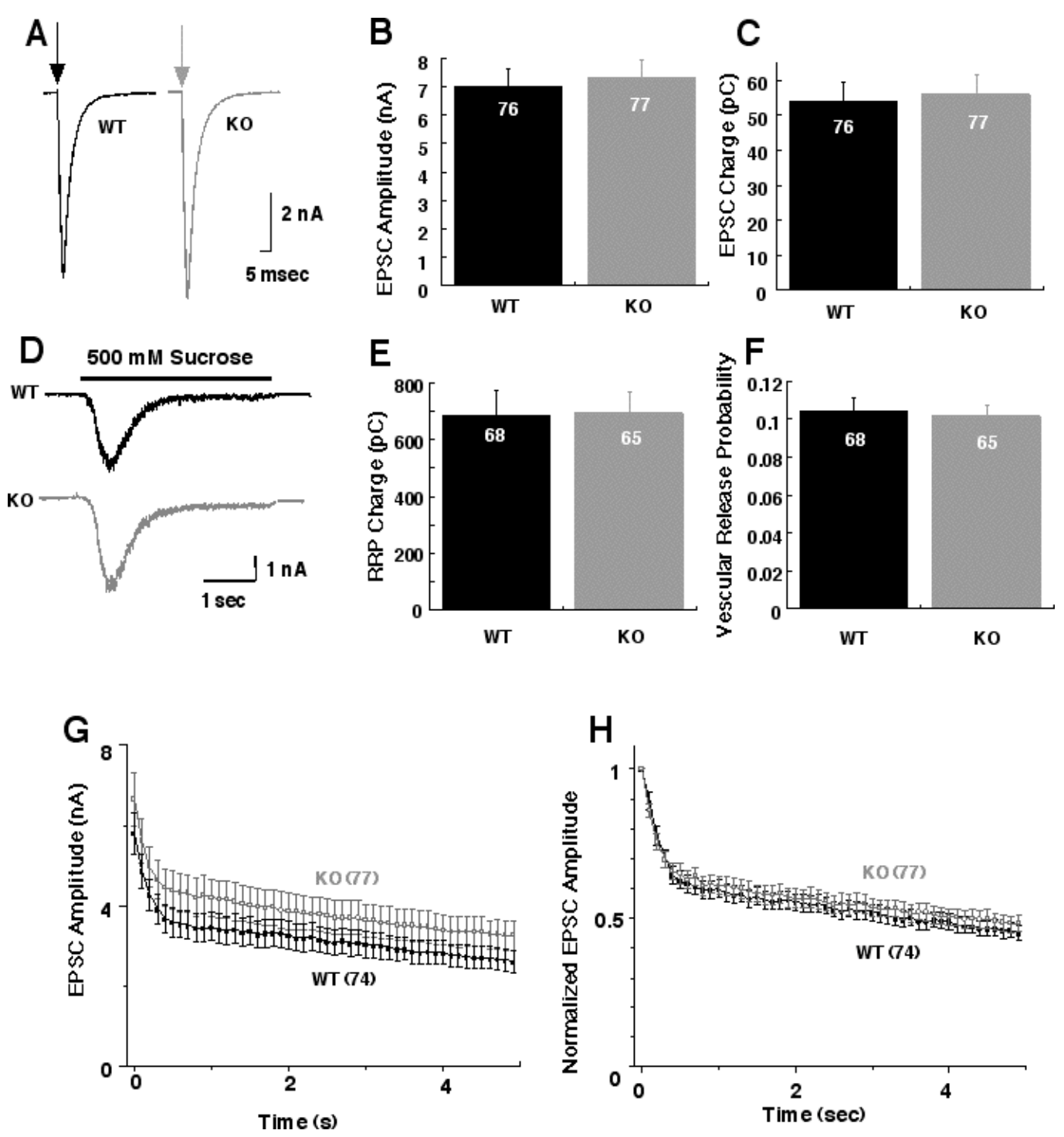

[Figure 9] Release efficacy and short-term plasticity of wild-type (WT) and syntaxin 1a knock-out (KO) neurons. Sample traces of wild-type (black) and Stx1AKO (gray) are shown in A. Arrows indicate the point of AP were evoked. Mean amplitude (B) and charge (C) of EPSC are compared between WT and KO in autaptic hippocampal neuronal culture. Neither charge nor amplitude of EPSC shows significance in difference (B, C). To determine the RRP size, $500 \mathrm{mM}$ sucrose was applied for $4 \mathrm{~s}$, and the transient component of inward current was integrated and determined as RRP (Rosenmund and Stevens, 1996). Examples of sucrose responses are shown in $\mathbf{D}$. Black bar above traces shows time the sucrose was applied. E shows bar plot of mean RRP charges of two groups. F, Vesicular release probability, as calculated from the ratio of charge released by a single action potential to the charge released by sucrose application, was not changes by the Stx1A. G, H, Change of EPSC during $10 \mathrm{~Hz}$ trains. The averaged actual amplitudes (G) and average normalized amplitude during tetanic stimulation $\mathbf{( H )}$. Both WT and KO amplitudes show approximately $50 \%$ depression, compared to the first of the train. Numbers in bars (B, C, E and F) and in blanket in G, H shows $n$. 


\subsection{Calcium Sensitivity of EPSC}

The sensitivity of EPSC amplitude to external $\mathrm{Ca}^{2+}$ is a parameter of importance, since evoked neurotransmitter release is tightly governed by the triggering molecule, $\mathrm{Ca}^{2+}$.

To access whether there is any basic change in the sensitivity to external $\mathrm{Ca}^{2+}$ concentration $\left(\left[\mathrm{Ca}^{2+}\right]_{\mathrm{ex}}\right.$ ), we used a simple experimental manipulation. Sensitivity to $\mathrm{Ca}^{2+}$ and saturation of release efficacy was monitored by measuring the level of potentiation of EPSC caused by increasing $\mathrm{Ca}^{2+}$ concentration in the external medium. As explained before (see part 2.8 and 3.4.4-4), the relationship between release probability and $\left[\mathrm{Ca}^{2+}\right]_{\mathrm{ex}}$ shows sigmoid shape. In other words, once release probability reaches plateau state, increasing $\left[\mathrm{Ca}^{2+}\right]_{\mathrm{ex}}$, henceforth, cannot cause any further increase in the release probability. Based on the principles of this relationship, manipulation of $\left[\mathrm{Ca}^{2+}\right]_{\mathrm{ex}}$ can be used for estimating release probability. The cells with high release probability will show less potentiation in response to elevation of $\left[\mathrm{Ca}^{2+}\right]_{\mathrm{ex}}$, because they are more saturated in terms of release probability. On the other hand, more potentiation is expected for the cell with initially low release probability.

External solution containing I2 $\mathrm{mM} \mathrm{Ca}{ }^{2+}$ and I $\mathrm{mM} \mathrm{Mg}{ }^{2+}\left(\mathrm{I}_{2} \mathrm{Ca}^{2+} / \mathrm{I} \mathrm{Mg}^{2+}\right)$ was applied, and the potentiation in EPSC was analyzed. To even out the run-down 
of amplitude, EPSC measuring in I2 $\mathrm{Ca}^{2+} / \mathrm{I} \mathrm{Mg}^{2+}$ was preceded and followed by standard external solution containing $4 \mathrm{mM} \mathrm{Ca}^{2+}$ and $4 \mathrm{mM} \mathrm{Mg}^{2+}$, at least for 60 . Mean amplitude in I2 $\mathrm{Ca}^{2+} / \mathrm{I} \mathrm{Mg}^{2+}$ was compared to average of control measurement before and after. Typical wild-type autapses show approximately 50\%$70 \%$ of potentiation in response to this manipulation.

I2 $\mathrm{Ca}^{2+} / \mathrm{I} \mathrm{Mg}^{2+}$ induced approximately I.6 fold potentiation of EPSC amplitude both in the W'T and StxiAKO groups ( $\mathrm{I} .6 \pm 0.3, \mathrm{n}=43$ for wild-type; $1.6 \pm$ O.I, $\mathrm{n}=38$ for StxiAKO, $p=0.567 \mathrm{I} ;$ Figure IO). This result indicates that $\mathrm{Ca}^{2+}$ sensitivity of neurotransmitter release machinery is not significantly altered, thereby, further corroborating the fact that the StxIAKO neurons show classical release efficacy. 

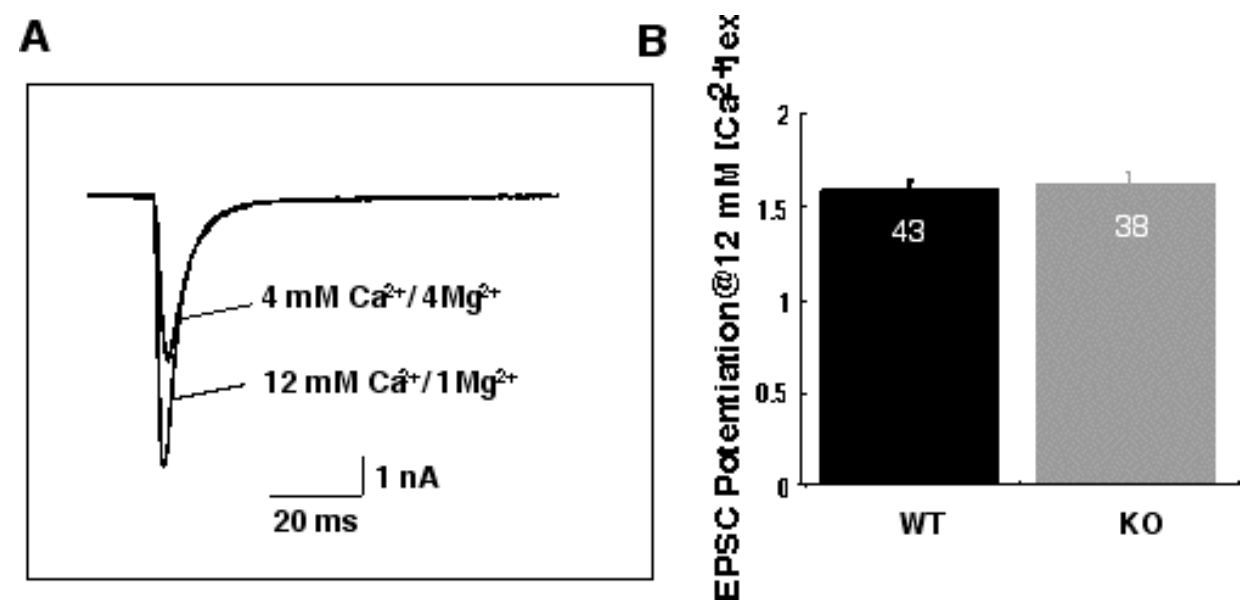

[Figure 10] EPSC potentiation by $12 \mathrm{mM}\left[\mathrm{Ca}^{2+}\right]_{\mathrm{ex}}$. Potentiation of EPSC by high $\mathrm{Ca}^{2+}$ concentration $\left(12 \mathrm{mM} \mathrm{Ca}^{2+}\right.$ and $1 \mathrm{mM} \mathrm{Mg}^{2+}$ ) was measured in comparison to EPSC in normal external solution containing $4 \mathrm{mM} \mathrm{Ca}^{2+}$ and $4 \mathrm{mM} \mathrm{Mg}^{2+}$. A, Representative raw traces for each condition $\mathbf{B}$, Averaged potentiation in EPSC, showing the same $\sim 1.6$ fold increase in both WT and KO groups. 


\subsection{Spontaneous Neurotransmitter Release in StxIAKO}

Another critical aspect of synaptic function is $\mathrm{Ca}^{2+}$-independent, spontaneous neurotransmitter release (mEPSC or minis). In spite of having no significant effect on the $\mathrm{Ca}^{2+}$ - dependent release properties such as vesicular release probability and short-term plasticity, the removal of StxiA from neurons could induce possible changes in the spontaneous activity. In principle, the amplitude and kinetics of minis provide information about postsynaptic responsiveness, charge of minis shows vesicular load or the quantal size, and the frequency of mini conveys information about $\mathrm{Ca}^{2+}$ independent release efficacy. In order to detect changes in any of those parameters as a consequence of the StxiA deletion, mEPSCs were recorded and analyzed. Recording of mini events was performed in the presence of $0.3 \mu \mathrm{M}$ tetrodotoxin (TTX) at holding potential of $-70 \mathrm{mV}$, which is close to neuronal resting membrane potential. The amplitude, charge and frequency of mini events were analyzed using template-based miniature detecting program.

No significant change was found in the amplitude $(\mathrm{WT}=24.6 \pm$ I.I pA, $\mathrm{n}=39: \mathrm{KO}=26.3 \pm \mathrm{I} .2 \mathrm{pA}, \mathrm{n}=40, p=0.4473)$, charge $(0 . \mathrm{IO} 6 \pm 0.006, \mathrm{n}=39$ for $\mathrm{WT}$; $0 . \mathrm{IIO} \pm 0.005, \mathrm{n}=4 \mathrm{O}$ for $\mathrm{KO}, p=0.33 \mathrm{I} 7)$, or frequency $(\mathrm{WT}=7.5 \mathrm{~Hz} \pm 0.7, \mathrm{n}=39$ and $\mathrm{KO}=6 . \mathrm{I} \pm 0.6, \mathrm{n}=40, p=0.155 \mathrm{I})$ of the mEPSC as shown in the Figure II.

Therefore, from this observation, it can be concluded that knocking out StxiA does not lead to any change in either evoked or spontaneous neurotransmitter release. 


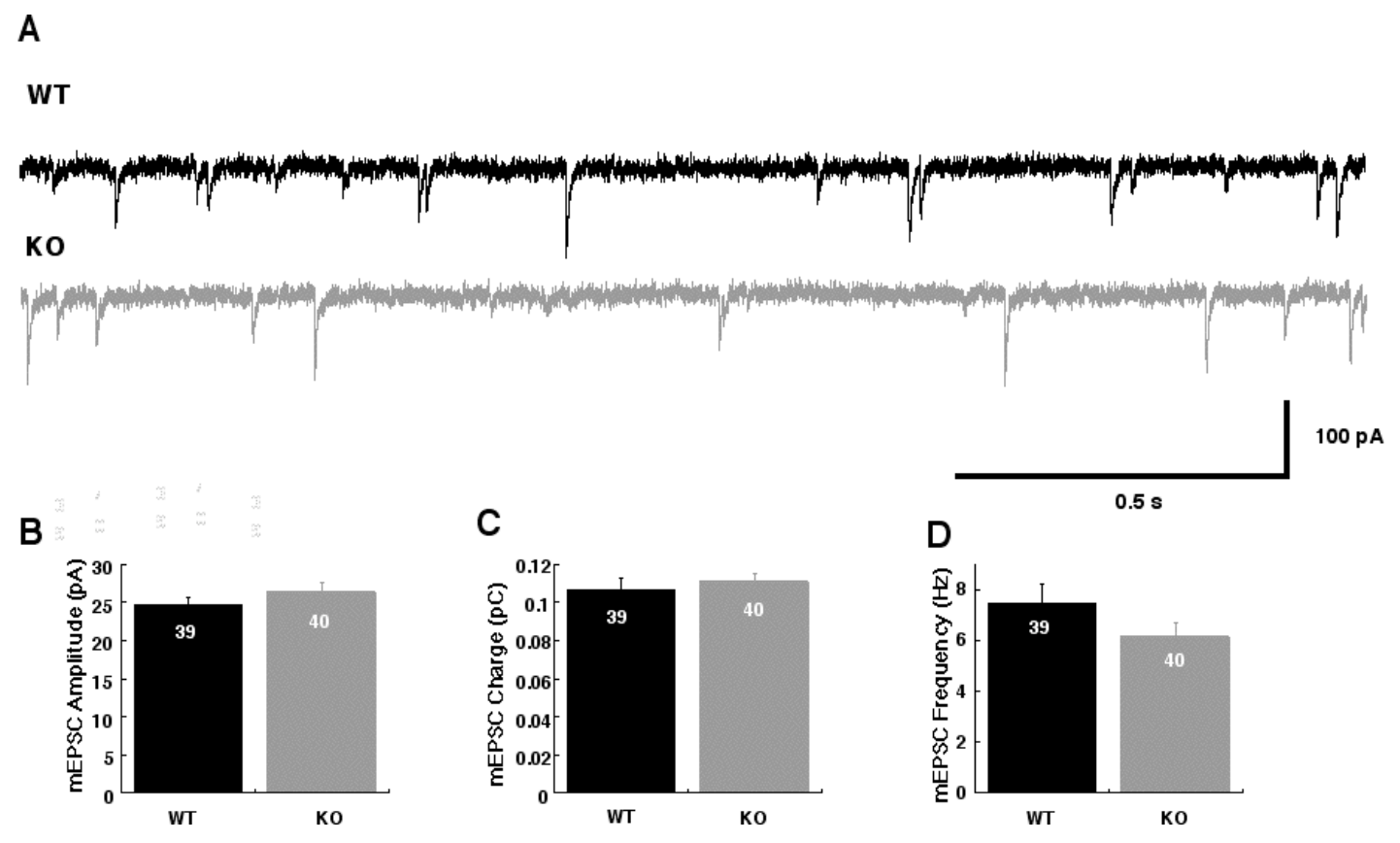

[Figure 11] Spontaneous neurotransmitter release in WT and Stx1AKO. A, Sample traces of miniature release in WT (black) and Stx1AKO (gray) neurons. To compare $\mathrm{Ca}^{2+}$ independent neurotransmitter release between WT and KO neurons, amplitude (B), charge (C) and frequency (D) of spontaneous neurotransmitter release were analyzed. Template shape based detection program was used for this analysis. Detected events were averaged in every cell for estimating charge and amplitude. None of the measured parameters vis-à-vis mean amplitude, charge or frequency shows significant alteration from wild type behavior. Numbers in bars show number of cells tested. 


\subsection{Turnover rate of readily releasable pool in StxIA KO}

It has been consistently found that the various parameters of vesicle fusion, vis-à-vis evoked response, RRP size, vesicular release probability, short-term plasticity, $\mathrm{Ca}^{2+}$-senstivity as well as spontaneous response remained unaffected by the removal of StxiA.

In view of these findings, attention was shifted to the pre-fusion stages, in order to study the effect of StxiA deletion on rate of the vesicular pool turnover, the efficiency of synaptic vesicle translocation, docking and priming to active zone of synapse. As described in experimental procedure, typically sucrose response is composed of a fast, transient component, which stands for the release of the RRP, followed by a sustained steady state component, which provides a measure for the refilling rate of the RRP.

Average refilling rate of RRP was determined by measuring the mean amplitude of the sustained component of the sucrose response. To quantify variance in the pool size of individual cells, mean amplitudes of the sustained components were normalized to the charge of the respective sucrose transients within the cell. Thus, the values show the turnover rate, refilled proportion of the RRP per unit time (in pool units/s) and the reciprocal of these values represent the time needed for refilling one pool unit. In the comparison between $\mathrm{W} T$ and StxiAKO, no difference 
was observed $\left(0.14 \pm 0.02\right.$ pool unit/s for $\mathrm{W}^{\prime} \mathrm{T}$ and $0 . \mathrm{I} 4 \pm 0 . \mathrm{OI}$ pool unit/s for StxIAKO, $p=0.5502$; Figure I2). This result of RRP turnover rate correlates well with the previously reported rate of W'T hippocampal autaptic neurons (Reim et al., 200I), implying that StxiA loss did not considerably affect the rate of vesicle translocation, docking and priming. 
A

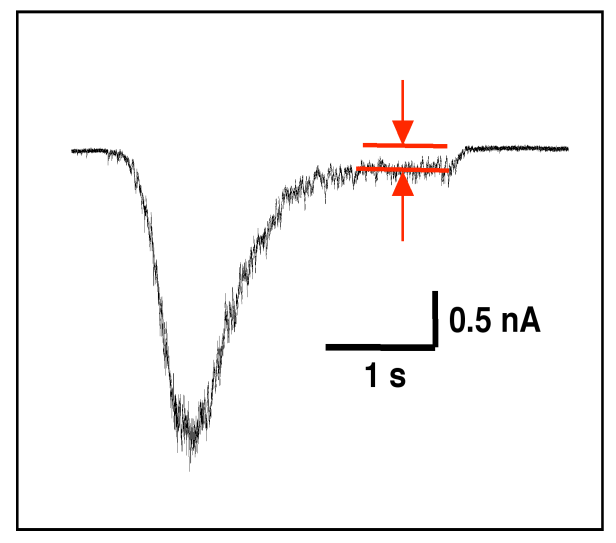

B

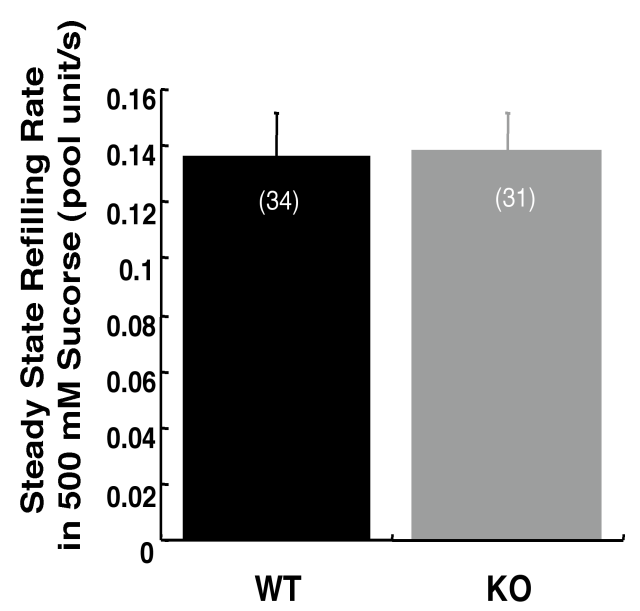

[Figure 12] Turnover rate of readily releasable pool. Sustained component of sucrose response stands for refilling rate of the RRP after depletion. A. Representative sucrose response and the way of measuring mean amplitude of sustained component (red arrows). B. Average of mean amplitude in sustained component of the response. Refilling rates obtained from the mean amplitude of sustained component show no difference between wild-type (WT) and knock-out (KO). Numbers in bars show the number of cells measured. 


\subsection{StxIA KO cells as a control for comparison with syntaxin Ib open-form} mutants

It has been establised through these studies comparing the fundamental parameters of neurotransmitter relase of the murine hippocamal StxIAKO neurons to their wild-type counterparts, that their phenotypes are essentially the same with respect to $\mathrm{Ca}^{2+}$-dependent as well as $\mathrm{Ca}^{2+}$-independent neurotransmitter release, $\mathrm{Ca}^{2+}$ sensitivity, and turnover rate of the RRP.

Therefore, we concluded that it is acceptable to regard neurons from StxiA $\mathrm{KO}$ mice as a control for analysis of double mutant mice with StxiB open-form mutation and StxiA knock-out $\left(\operatorname{StxiB}_{\text {of }}\right)$.

\section{Io Basic Characteristics of double mutant mice expressing constitutively open conformation of syntaxin $\mathrm{Ib}$ and syntaxin Ia null $\left(\mathrm{StxI} B_{o f}\right)$.}

Stxi has been implicated as the only SNARE component, which switches to open-conformation, exposing its core complex region, so as to regulate the actual formation of SNARE complex (Dulubova et al., I999). This implies that syntaxin is the key molecule that controls the critical step of membrane fusion.

However, in spite of the importance of the molecule in regulation of neurotransmitter release, the functional significance of its conformational switching, 
by which it actually exercises this regulation remains poorly understood (Dulubova et al., 1999; Jarvis et al., 2002). In the present study, characterization of double mutant mice expressing constitutively open conformation of StxiB, lacking StxIA $\left(\mathrm{StxIB}_{\mathrm{of}}\right)$ was carried out to address this crucial issue.

$\mathrm{StxiB}_{\text {of }}$ mice were provided by S.Gerber and T.Südhof and maintained as heterozygotes. At birth $\mathrm{Stxi}_{\text {of }}$ homozygote mice had similar body weight compared to control but usually developed seizure at the onset age of two weeks, and eventually died approximately a month after birth. However, the $\mathrm{Stxi}_{\text {of }}$ mice did not display significant abnormalities in gross anatomy, or brain structure (S.Gerber and T.Südhof, unpublished).

\section{II Characterization of Vesicular Release efficacy in $\operatorname{StxI} B_{\text {of }}$}

In hippocampal autaptic neurons from $\mathrm{StxIB}_{\text {of }}$, amplitude and charge of EPSC, RRP were measured and vesicular release probability was calculated in the same manner as in the StxiA null study.

Mean amplitude and charge of EPSCs from $\mathrm{StxIB}_{\text {of }}$ and StxIA KO (control) of the same age in vitro show no significant difference (Figure $13 \mathrm{~A}, \mathrm{~B}$, and $\mathrm{C}$; Measured EPSCs amplitude were $7.7 \pm 0.4 \mathrm{nA}, \mathrm{n}=\mathrm{I} 7 \mathrm{I}$ for control; $7.8 \pm 0.4 \mathrm{nA}$, $\mathrm{n}=185$ for $\mathrm{StxiB}_{\text {of }}, p=0.9824$ and EPSCs charge were $57 \pm 4 \mathrm{pC}$ and $57 \pm 4 \mathrm{pC}$ for control and $\mathrm{Stxi}_{\mathrm{of}}, p=0.6802$ ). These results indicate that basic machinery for 
membrane docking and fusion are not destroyed by constitutive opening of StxiB and time course of EPSC was not dramatically changed.

However, the open-form mutation in StxiB causes reduction of RRP size to half of the control; $480 \pm 70 \mathrm{pC}, \mathrm{n}=\mathrm{I} 47$ in $\mathrm{StxIB}_{\text {of }}$ compared to $760 \pm 60 \mathrm{pC}, \mathrm{n}=\mathrm{I} 67$ for control: $p<0.000 \mathrm{I}$ ). Thus, vesicular release probability of $\mathrm{StxiB}_{\text {of }}$ becomes approximately 2.2 fold as high as control, because this is the ratio of constant EPSC charges to half-reduced RRP ( $\mathrm{Pvr}=8.9 \pm 0.4 \%, \mathrm{n}=\mathrm{I} 46$ for control; $19.7 \pm 0.9 \%$, $\mathrm{n}=\mathrm{I} 67$ for $\mathrm{StxiB} \mathrm{B}_{\mathrm{of},} p<0.000 \mathrm{I}$ : see figure $\mathrm{I}_{3} \mathrm{D}$ and $\left.\mathrm{F}\right)$

\section{I2 Short-term plasticity of $S t x i B_{\text {of }}$}

As described above, short-term plasticity is a very useful tool for comparison of release probability. To confirm the increased vesicular release probability in $\mathrm{StxIB}_{\text {of }}$, synaptic amplitudes during trains of action potentials at frequencies of $\mathrm{IOHz}$ and $20 \mathrm{~Hz}$ were compared. The short-term plasticity of $\mathrm{StxI}_{\text {of }}$ showed dramatically faster depression and greater depletion in both frequencies of trains (Figure I4), confirming the increased vesicular release probability in $\mathrm{StxI}_{\text {of }}$ neurons (Figure I3). 


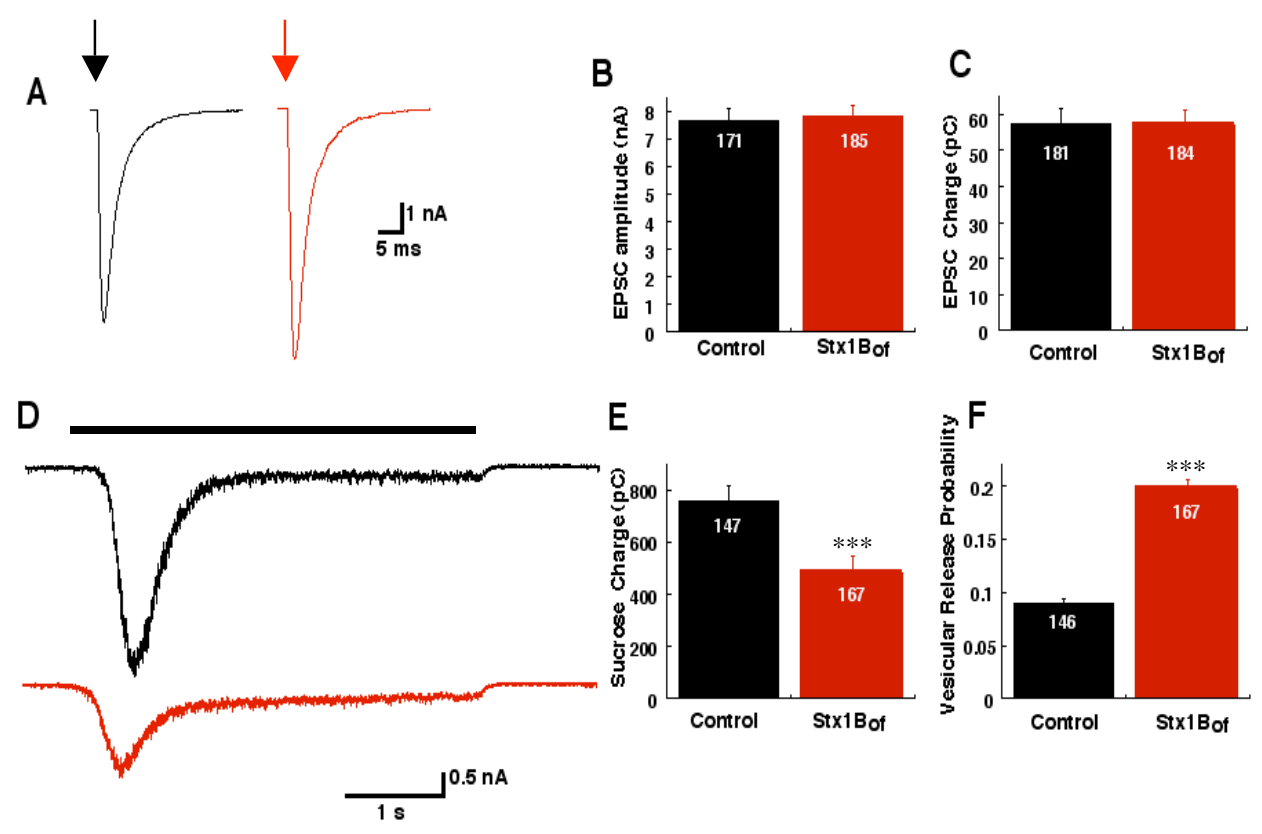

[Figure 13] Vesicular release probability of syntaxin 1b open-form mutant $\left(\mathrm{Stx}_{1 \mathrm{~B}_{\mathrm{of}}}\right)$. A, Sample traces of evoked postsynaptic responses for control (black) and syt1 $\mathrm{B}_{\text {of }}$ (Red). Arrows indicate the point AP was evoked. B,C, While EPSC of Stx $1 B_{\text {of }}$ is the same in amplitude and charge with syntaxin 1a knock out cell (control). D, Raw traces of sucrose reponse for controal and $\mathrm{Stx}_{1 \mathrm{~B}}$ of (black, red respectively). Black bar above traces represents application of hypertonic sucrose solution. E, The charge released by $500 \mathrm{mM}$ sucrose (RRP) was reduced approximately by half ${ }^{* * *} p<0.0001$. F, Thus, the release probability, calculated by these two factors, is increased approximately two folds. Number in each column shows number of cells tested, and error bars show S.E.M. ${ }^{* * *} p<0.0001$ 
A

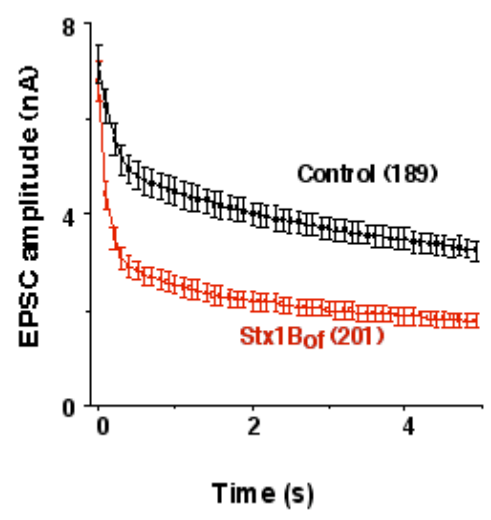

C

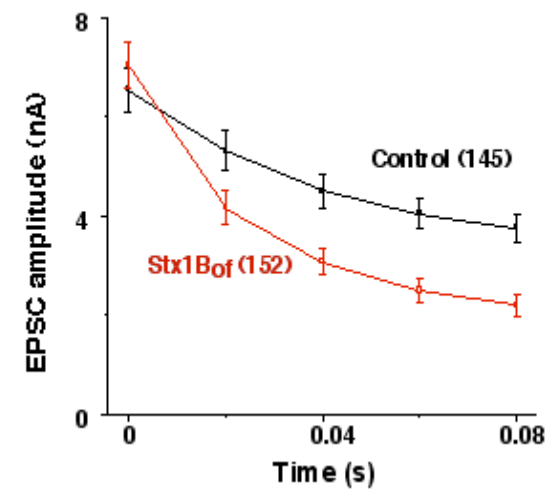

B

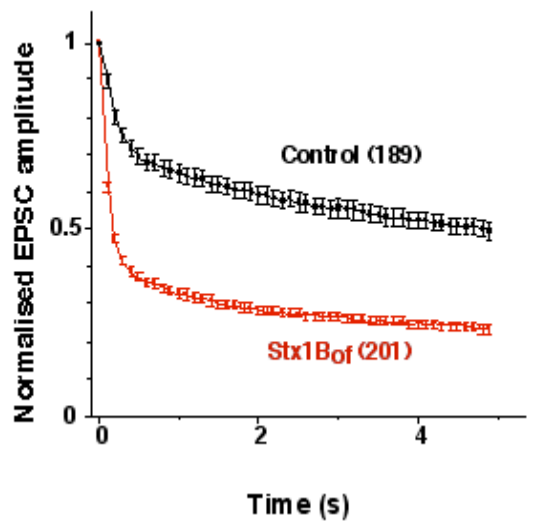

D

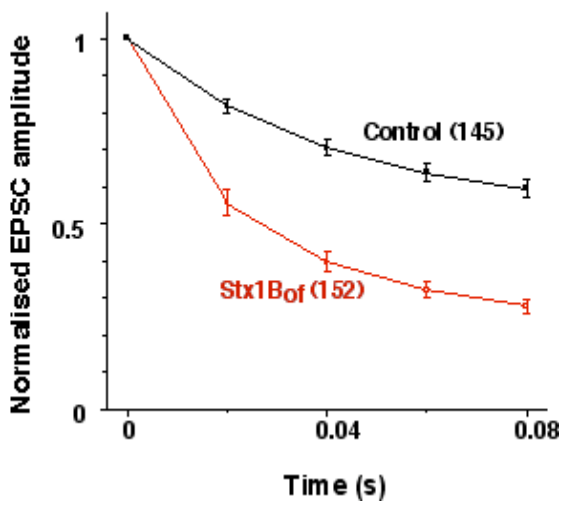

[Figure 14] Short-term plasticity characteristics of Stx $1 B_{\text {of }}$ Synaptic amplitudes during trains of action potential at frequencies of $10 \mathrm{~Hz}(\mathbf{A})$ and $20 \mathrm{~Hz}$ (C) were observed. EPSC amplitudes by tetanic stimulation were normalized to the one by the first stimulus in $10 \mathrm{~Hz}$ (B) and $20 \mathrm{~Hz}$ (D). In both trains, Stx $1 B_{\text {of }}$ shows dramatically faster depression, as well as the greater depletion, in contiguity with increased vesicular release probability. Numbers in blanket show number of cells tested and error bars show S.E.M. 


\section{I3 Synaptic release probability}

So far, we have shown the probability of individual vesicle to fuse by an action potential, so called, vesicular release probability is increased in StxiB $B_{\text {of }}$ neurons. Also, it was revealed that overall synaptic response was not changed and RRP size was rather decreased by the mutation. Since synaptic release probability is proportional to two different factors, vesicular release probability and number of vesicles in RRP (Dobrunz and Stevens, 1997), our results are indicating that both factor that increases efficiency as well as decrease efficiency of synaptic transmission are occurring with open-form mutation of StxiB. To analyze the efficiency using completely independent method that depends on synaptic output, we used MK-80I method.

The underlying principle of this technique is based on the irreversible block of postsynaptic open NMDA receptors by MK-80I. The rate at which synaptic NMDA-EPSCs are blocked is dependent upon the probability that any vesicle at given synapses is released during an action potential. Thus, the higher the synaptic release probability, the faster is the MK80I induced blockage of the NMDA-EPSC (Rosenmund et al., I993). 
To measure the NMDA mediated EPSCs, extracellular solution was modified. $\mathrm{Mg}^{2+}$ was removed, $\mathrm{Ca}^{2+}$ was decreased to $2.7 \mathrm{mM}$ and glycine was added at a concentration of Io $\mu \mathrm{M}$ (Rosenmund et al., I993). Under this condition, we can simultaneously measure the AMPA-mediated and the NMDA-mediated EPSC. The AMPA mediated EPSC was the fast component of dual component of EPSC, while the NMDA mediated EPSC was slower component. After initial measurement of EPSC period, the extracellular solution was switched to the solution containing $5 \mu \mathrm{M}$ MK-8oI. The dual components of EPSCs were recorded during IOO consecutive action potentials and we used AMPA receptor-dependent fast component as internal normalization factor for possible presynaptic run-down of the EPSC.

When we compared the normalized NMDA decay constant for $\mathrm{StxIB}_{\text {of }}$ with control, we noticed that initial, rapid phase of NMDA component decay was slightly faster but not quite significant in $\mathrm{StxIB}_{\text {of }}$ than in control $(\tau=3.5 \pm 0.2$ stimuli, $\mathrm{n}=\mathrm{I} 6$ for control and $3.0 \pm$ o.I stimuli for $\left.\mathrm{StxIB}_{\text {of }} \mathrm{n}=2 \mathrm{O} ; p=0.072 \mathrm{I}\right)$ and ultimately, come to the same level during the later slow decay phase. This result was consistent with heterogeneous synaptic release probability in hippocampal autaptic cultures (Rosenmund et al., I993). Also, our data indicate that the increase in vesicular release probability was compensated by decrease in number of fusion-competent vesicles in RRP keeping the overall synaptic output at low frequency stimulation relatively stable. 
Based on our findings (A) synaptic output remains unchanged, (B) synaptic release probability is almost not changed; we can conclude that the increase in vesicular release probability has been compensated by the decrease in number of vesicles in the RRP. These result is further supported by faster depression and greater depletion of EPSC during trains of action potentials because of the reduced number of vesicles per active zone. 

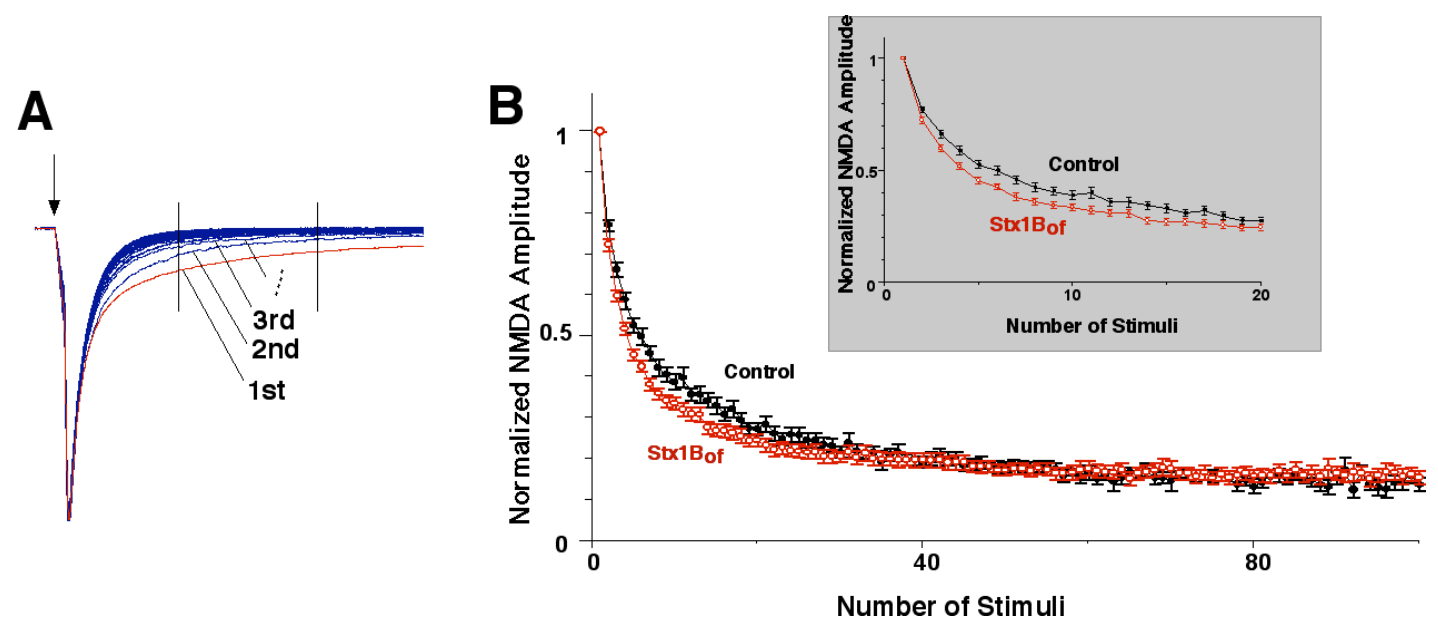

[Figure 15] Determining Synaptic release probability as given by the decay of NMDA ( $\mathrm{N}$-methyl-D-aspartate) dependent current in the presence of MK-801. Dual components of AMPA- and NMDA-mediated were activated every $5 \mathrm{sec}$ by somatic depolarization with $10 \mu \mathrm{M}$ glycine and $2.7 \mathrm{mM}\left[\mathrm{Ca}^{2+}\right]$ in the absence of external $\mathrm{Mg}^{2+}$. Amplitudes of NMDA component were measured during consecutive 100 stimuli at every $5 \mathrm{sec}$ in presence of $5 \mu \mathrm{M}$ MK- 801 . During the successive stimulation, activated proportion of the channel is shown as decay. NMDA currents were determined as mean amplitude between 50$100 \mathrm{~ms}$, in most cases. In the cells that have big AMPA current, time window was shifted accordingly. A, Sample traces of NMDA current during spike train with MK-801. Arrow shows the point where action potential was evoked. AMPA component of EPSC was used as internal control of presynaptic run-down B, Average normalized NMDA EPSC during 100 stimuli. At the beginning of stimulation, $\mathrm{Stx}_{1 \mathrm{~B}_{\text {of }}}$ shows slightly faster but not significant decay in NMDA current and ultimately, reach same level during the later slow decay phase $(p=0.7619)$. First 20 responses were magnified in inset. 


\section{I4 Calcium sensitivity to neurotransmitter release}

Our results so far lead to the conclusion that constitutive opening of StxiB leads to an increase in the release probability of vesicles. One possible explanation of this increased vesicular release probability could be that the calcium sensitivity of the release apparatus was increased by the open-form mutation of StxiB, alternatively, the mutation induced greater calcium influx. To evaluate these possibilities, first, EPSC amplitude was measured in the varying external concentrations of calcium (I, 2, 4, 8 and $\mathrm{I} 2 \mathrm{mM}$ ), holding magnesium at constant concentration (I mM). Each mean EPSC at the testing concentrations was normalized to the mean EPSC amplitude under the standard concentration $\left(4 \mathrm{mM}\left[\mathrm{Ca}^{2+}\right]_{\mathrm{ex}}, 4 \mathrm{mM}\left[\mathrm{Mg}^{2+}\right]_{\mathrm{ex}}\right.$; see figure 6)

As shown in [Figure I6], as compared to the control (StxIAKO), EPSC amplitudes of $\mathrm{StxI}_{\text {of }}$ were less reduced at lower concentrations of external calcium (EPSC of control and $\mathrm{StxiB}_{\text {of }}$ at I $\mathrm{mM}\left[\mathrm{Ca}^{2+}\right]_{\mathrm{ex}}$ was respectively $0.36 \pm 0.03(\mathrm{n}=\mathrm{I} 6)$ and $0.52 \pm 0.04(\mathrm{n}=\mathrm{I9})$ of EPSC in standard condition $(p=0.0 \mathrm{IO}))$. Also, a smaller degree of potentiation was observed in $\mathrm{StxIB}_{\text {of }}$ at $\mathrm{I} 2 \mathrm{mM}\left[\mathrm{Ca}^{2+}\right]_{\mathrm{ex}}$, EPSC of control potentiated I.70 $\pm 0.09\left(\mathrm{n}=\mathrm{I}_{5}\right)$ while $\mathrm{StxIB}_{\text {of }}$ showed I.38 $\pm 0.04\left(\mathrm{n}=\mathrm{I}_{5} ; p=0.0 \mathrm{I}_{4}\right)$

As a consequence, the overall relationship between EPSC and $\mathrm{Ca}^{2+}$ - dose showed an obvious left shift. However, this apparent left shift is not necessarily 
indicative of increased $\mathrm{Ca}^{2+}$ sensitivity of release apparatus but it also can interpreted by the overall increase in $\mathrm{Ca}^{2+}$-independent release probability (see part $3 \cdot 4 \cdot 4-4$ and $4 \cdot 16)$.

\section{I5 Somatic Calcium Current}

Next, to analyze whether the mutation was directly affecting $\mathrm{Ca}^{2+}$ regulating function of Stxi, we assessed $\mathrm{Ca}^{2+}$ channel function. The possible alteration in $\mathrm{Ca}^{2+}$ regulation by open-mutation of Stxi has been suggested by some previous reports that voltage-gated calcium channels are regulated by Stxi and this regulation depends on conformations of Stxi (Jarvis and Zamponi, 200I). Since our culture system does not allow for direct recording of presynaptic terminal because of its small size, we analyzed $\mathrm{Ca}^{2+}$ current at soma. It is known that the soma contains similar types of channels that are present at the presynapse (N- and P/Q-types) and also Stxi is present at the plasma membrane of soma.

To record $\mathrm{Ca}^{2+}$ current specifically, voltage-dependent conductance of $\mathrm{Na}^{+}$ and $\mathrm{K}^{+}$was blocked by using Cs-based internal solution and external solution containing $0.5 \mu \mathrm{M}$ TTX and IO mM TEA. We measured somatic $\mathrm{Ca}^{2+}$ current by depolarizing the neuronal somatic membrane from $-70 \mathrm{mV}$ to $\circ \mathrm{mV}$ for Io msec. In addition, to remove L-type $\mathrm{Ca}^{2+}$ channel-dependent conductance, which contributes little at neurotransmitter release, we added L-type $\mathrm{Ca}^{2+}$ channel antagonist, 
nifedipine $(3 \mu \mathrm{M})$ to the extracellular solution.

When we averaged the mean somatic calcium currents with and without Ltype channel contribution in the control and $\mathrm{Stxi}_{\text {of }}$ neurons, we found no significant difference. $I_{\mathrm{ca}}$ with L-type channel contribution was $0.66 \pm 0 . \mathrm{I} O \mathrm{nA}, \mathrm{n}=\mathrm{I} 5$ for control; $0.65 \pm 0.07 \mathrm{nA}, \mathrm{n}=\mathrm{I} 6$ for $\mathrm{StxIB}_{\mathrm{of}}, p=0.6213$ and $\mathrm{I}_{\mathrm{ca}}$ without L-type channel contribution was $0.40 \pm 0.05(n=I 2)$ for control and $0.40 \pm 0.07(n=I I)$ for $\mathrm{StxIB}_{\text {of }}$, respectively $(p=0.9274)$. Even though further studies on presynaptic $\mathrm{Ca}^{2+}$ current are needed for a definite conclusion, a possible putative change in presynaptic $\mathrm{Ca}^{2+}$ current is unlikely to be the main cause of a 2.2 fold increase in the vesicular release probability and the left shift of the apparent sensitivity of the EPSC to external $\mathrm{Ca}^{2+}$ concentration [Figure i 7 ]. 


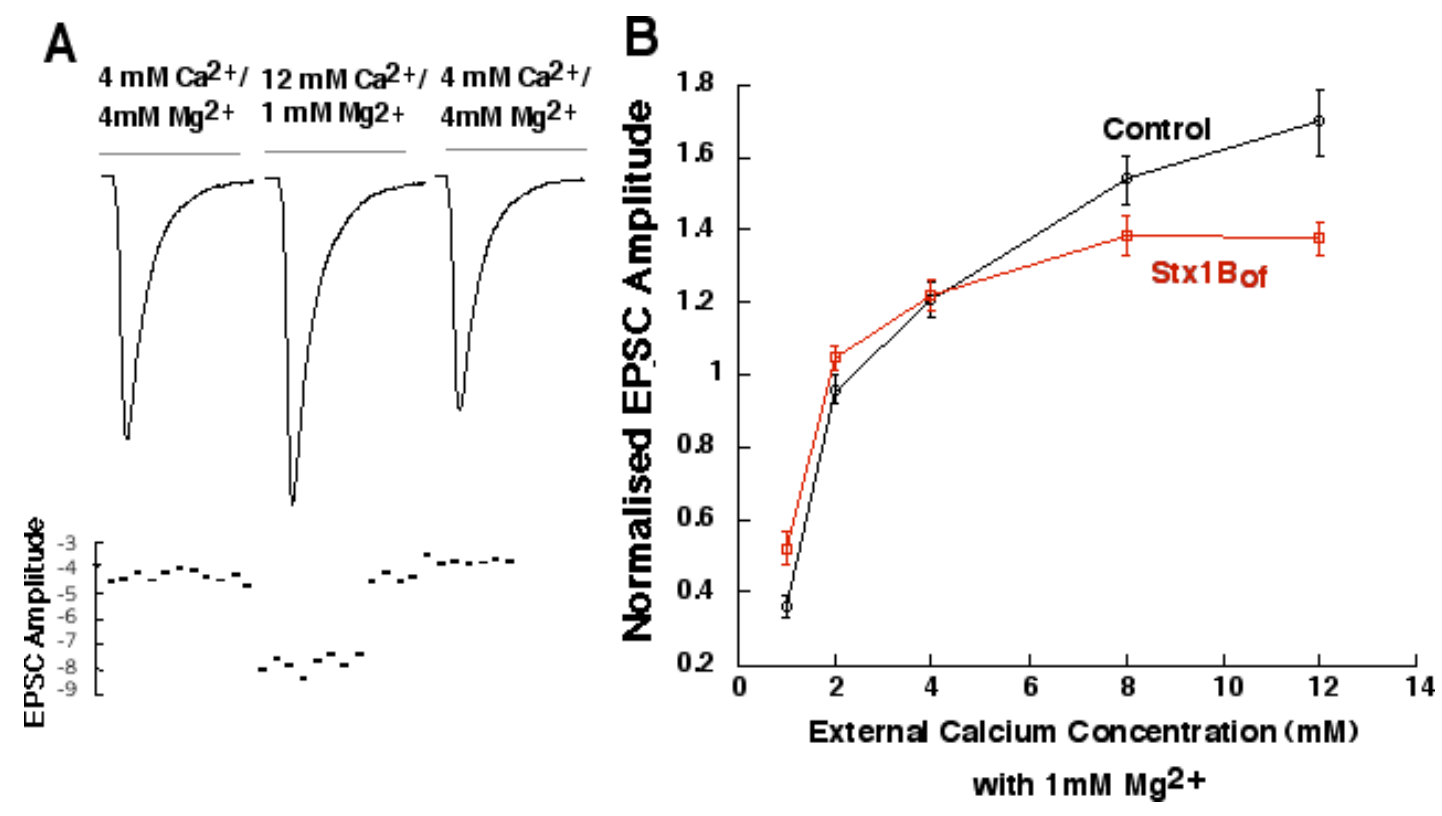

[Figure 16] Dependency of EPSC amplitude upon external $\mathrm{Ca}^{2+}$ concentration. EPSC amplitudes were measured at $0.2 \mathrm{~Hz}$ at various concentration of external calcium (1-12 mM), with constant $1 \mathrm{mM} \mathrm{Mg}^{2+}$. To compensate the rundown effect and variability of EPSC between cells, we scaled EPSC in standard condition ( $4 \mathrm{mM} \mathrm{Ca}^{2+}, 4 \mathrm{mM} \mathrm{Mg}^{2+}$ ) before and after the individual test recording. The responses at various concentrations of external $\mathrm{Ca}^{2+}$ were normalized to EPSC's under standard condition. Note that responses in $\mathrm{Stx}_{1 \mathrm{~B}}$ of less increase at lower $\left[\mathrm{Ca}^{2+}\right]_{\mathrm{ex}}$ and also decreased less at higher $\left[\mathrm{Ca}^{2+}\right]_{\mathrm{ex}}$ in comparison to $\mathrm{KO}$ group. Change of normalized EPSC by $1 \mathrm{mM}$ and $12 \mathrm{mM} \mathrm{Ca}^{2+}$ showed significant difference $p=0.0103$ and 0.0114 , respectively. Error bars show S.E.M. 


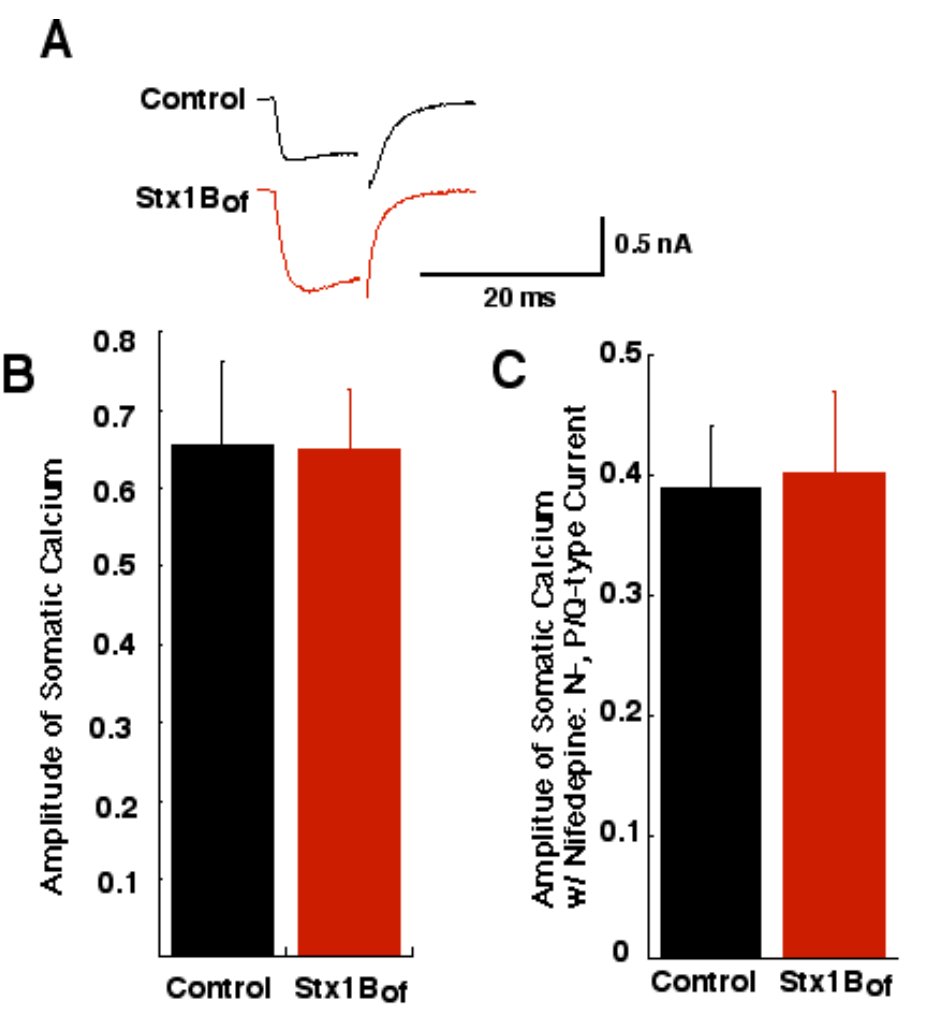

[Figure 17] Somatic $\mathrm{Ca}^{2+}$ current comparison between control and Stx1 $\mathrm{B}_{\text {of }}$ neurons. To measure somatic $\mathrm{Ca}^{2+}$ current, cells were depolarized for $10 \mathrm{~ms}$ to $0 \mathrm{mV}$ in presence of internal Cs and external $0.5 \mu \mathrm{M}$ TTX and $10 \mathrm{mM}$ TEA A, Leak subtracted sample trace of each group. Total somatic $\mathrm{Ca}^{2+}$ current as well as the amplitude L-type dependent current subtracted by $3 \mu \mathrm{M}$ Nifedipine. B, No significantly different amplitude of the currents were observed in both with and without L-type $\mathrm{Ca}^{2+}$ channel conductance. 


\section{I6 Spontaneous Neurotransmitter Release}

So far our data indicates that the open-form mutation of StxiB leads to an enhanced vesicular release probability, in addition to a shift of EPSC sensitivity to external $\mathrm{Ca}^{2+}$. These data could mean that overall sensitivity to $\mathrm{Ca}^{2+}$ is increased; alternatively, this could be the consequence of a shift in the $\mathrm{Ca}^{2+}$-independent release probability, which is not dependent on $\mathrm{Ca}^{2+}$ sensing (see 3.4.4-4). One way of resolving these possibilities would be to test whether spontaneous neurotransmitter release is affected by the open-form mutation. If we observe the enhanced spontaneous release frequency, which is by definition a $\mathrm{Ca}^{2+}$-independent neurotransmitter release, we would gain evidence for more efficient $\mathrm{Ca}^{2+}$ independent fusion of vesicles. In addition, it can be also interpreted as a reduced energy barrier for the fusion process itself. This analysis also conveys information about the possible changes in postsynaptic receptor responsiveness or loading of neurotransmitter into vesicles. Hence, we analyzed amplitude, charge, and frequency of miniature EPSCs in StxiB ${ }_{\text {of }}$ neurons as well as StxiAKO control neurons in the presence of $0.3 \mu \mathrm{M}$ TTX to eliminate any action potential or spontaneous $\mathrm{Ca}^{2+}$-driven release.

Our analysis of mEPSC between the two groups showed that neither average amplitude nor charge of mini events was significantly different between 
control and $\mathrm{Stxi}_{\text {of }}$ neurons, showing that constitutive opening of StxiB does not affect post synaptic or quantal parameters. The mean amplitudes of mEPSCs were $23 \pm$ I $\mathrm{pA}, \mathrm{n}=69$ for control and $25 \pm \mathrm{I} \mathrm{pA}, \mathrm{n}=68$ for $\operatorname{StxiB}_{\text {of }},(p=0.1235)$; and the mean charge of mEPSC were $0.106 \pm 0.005 \mathrm{pC}, \mathrm{n}=69$ for control and $0.094 \pm 0.005$ for $\operatorname{StxiB}_{\text {of }}(p=0.1704$; Figure I8 B and $\mathrm{C})$.

However, the average frequency of the spontaneous neurotransmitter release was significantly increased in $\mathrm{StxiB}_{\text {of }}$ neurons (Figure I8D: $5.3 \pm 0.5 \mathrm{~Hz}, \mathrm{n}=70$ for control, $7 \cdot 3 \pm 0.6 \mathrm{~Hz}, \mathrm{n}=68$ for $\left.\mathrm{StxiB}_{\text {of }}, p=0.0099\right)$. Since we measured mEPSC frequencies and RRP size in the same neuron, we could determine the spontaneous vesicular release probability from these neurons. The 'spontaneous vesicular release probability' was calculated by dividing the frequency of mini events by the number of vesicles in RRP. Because RRP size of $\mathrm{StxI}_{\text {of }}$ neurons was significantly reduced, we observed dramatic increase in probability of spontaneous release. The calculated spontaneous release probabilities were for control $0.0015 \pm 0.0004 \mathrm{~s}^{-1}(\mathrm{n}=62)$ and $0.0033 \pm 0.0004 \mathrm{~s}^{-1}(n=62)$ in $\operatorname{StxiB}_{\text {of }}$ neurons $(p<0.000 \mathrm{I})$ as shown in Figure I8 E. The level of increase in spontaneous release probability was approximately as large as the increase in the vesicular release probability of evoked responses $(2.2$ fold increase in both vesicular release probability as well as spontaneous release probability). Based on this finding, there is a possibility that the increased vesicular release probability of evoked responses may be caused, at least in large part, by this enhanced $\mathrm{Ca}^{2+}$-independent neurotransmitter release efficiency. 
A Control

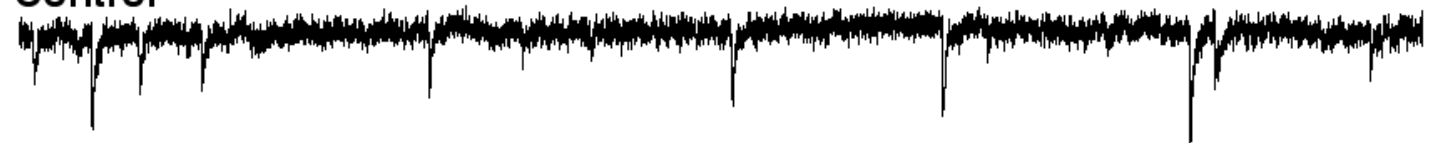

\section{Stx1Bof}
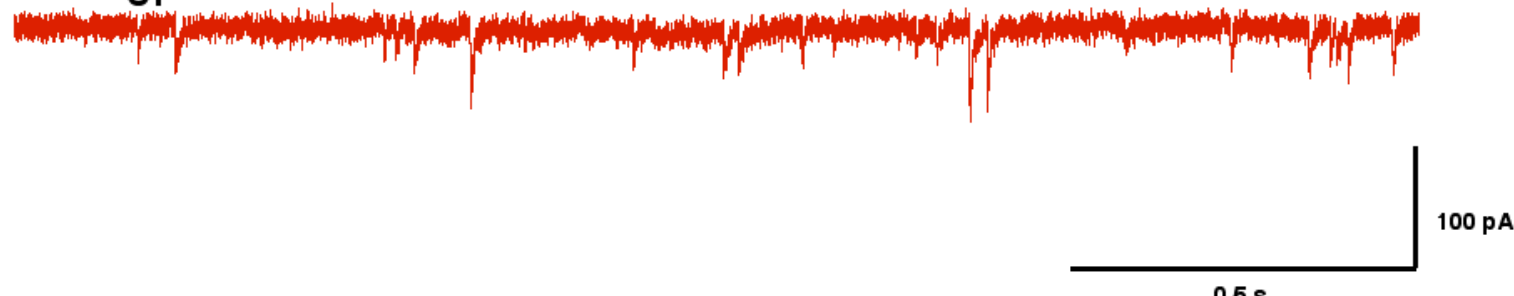

B

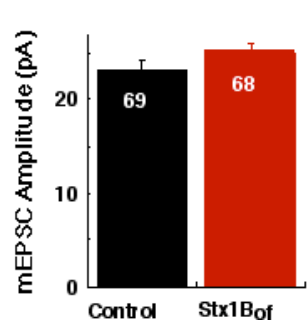

C

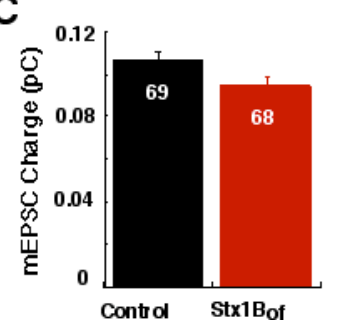

D

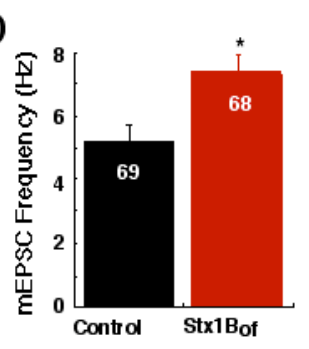

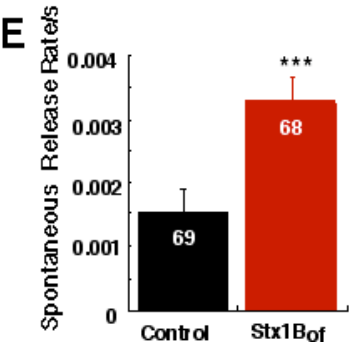

[Figure 18] Spontaneous neurotransmitter release efficiency in Stx1B of neurons. Spontaneous neurotransmitter release was measured at $-70 \mathrm{mV}$, in presence of $0.3 \mu \mathrm{M}$ TTX. A, Black and red traces represent sample trances for spontaneous neurotransmitter release of control and Stx $1 \mathrm{~B}_{\text {of }}$ neurons respectively. B, C, While amplitude and charge of mini events remain unchanged $\mathbf{D}$, frequency of spontaneous events was significantly increased in Stx1B $\mathrm{B}_{\text {ff }} \mathrm{E}$. To compare the frequency of events per number of vesicles in RRP, we calculated spontaneous release probability as the frequencies of mini events divided by the number of vesicles in RRP in each cell. Note that the spontaneous release probability was increased as much as vesicular release probability (2.2 fold). ${ }^{*} p<0.05$, ${ }^{* * *} p<0.0001$, Numbers in bars show the number of cells tested. 


\section{I7 Neurotransmitter release time course by osmotic pressure}

To test the hypothesis that the increased efficiency of spontaneous release is responsible for that of evoked responses, we systematically analyzed the time course of neurotransmitter release by osmotic pressure in more detail. Since hypertonic solutions are known to release the fusion-competent vesicles in a $\mathrm{Ca}^{2+}$ independent manner, if this hypothesis is correct, then vesicles would be released with greater ease. In other words, when the same amount of force, (for example, osmotic pressure) is applied to the synapse, a reduction in the energy threshold for neurotransmitter release, as may be the case for the $\mathrm{StxI}_{\text {of }}$, would cause an earlier onset and faster release of vesicles.

Firstly, to have a preliminary idea for the kinetics of the hypertonicityinduced response from each group, every trace was aligned at the peak point of the response and averaged to compare their width as described in the Experimental Procedure section. As expected, the responses of $\mathrm{StxiB}_{\text {of }}$ neurons are narrower than control (Figure I9A). Then, to retrieve the information altered during the trace alignment process, concerning onset of the response, we measured the time point of onset of the responses without alignment from individual cells. Since the onset of the responses varies depending on the geometry of the cell and overgrowth of the astrocyte, responses with longer than Is of time-to-onset were excluded in this study. 
The analysis showed that timing of the onset of hypertonic response was significantly decreased from $0.59 \pm 0.02 \mathrm{~s}(\mathrm{n}=53)$ to $0.52 \pm 0.02 \mathrm{~s}(\mathrm{n}=68)$ by the open mutation $(p=0.0318)$. Subsequently, knowing the onset time, we adjusted the positioning of the averaged sucrose responses according to measured onset time to directly compare the time course of the sucrose responses. As depicted in figure I9A, our preliminary results suggested possible changes in half-width and rise time. In addition, we noticed that sucrose response of $\mathrm{Stxi}_{\mathrm{of}}$ has greater inward steady state component, which is indicative of faster refilling. This will be dealt with in the priming rate of vesicles section.

For allowing estimation in terms of pool unit released per given time (rate of release of PU), we integrated transient components of these averaged responses and then normalized by the total charge of the RRP (Figure I9B). As shown in figure I9 $\mathrm{B}$, release by sucrose in $\mathrm{StxI}_{\text {of }}$ appears to have decreased time for complete release.

The maximum slope of the sucrose transient integral, which basically represents the release activity at the peak of the response, directly indicates release rate in pool unit/s caused by a constant degree of osmotic pressure. From this estimation, control neurons had I.699 pool unit/s, while Stxi $_{\text {of }}$ neurons had 2.275 pool unit/s for $\mathrm{StxiB}_{\text {of }}$, implying that there may be approximately a $30 \%$ increase in the maximum release rate in response to hypertonic sucrose. 
Next, we analyzed hypertonic solution (500 mM sucrose)-evoked responses from individual cells. In order to detect any change in the kinetics of the response, we analyzed four parameters from each sucrose transient: time-to-peak, half width, IO-90\% rise time and time to onset of the transient sucrose response. The responses with values lying outside of three times standard deviation in any parameter were not considered in this study. As expected by an average sucrose response curve in figure I9A, sucrose responses of $\mathrm{StxiB}_{\text {of }}$ showed narrower transients and required less time to reach the peak of release (figure ${ }_{19} \mathrm{C}$ ). Time-to-peak was significantly reduced from I.OI $\pm 0.03 \mathrm{sec}(\mathrm{n}=53)$ to $0.9 \mathrm{I} \pm 0.02 \mathrm{sec}(\mathrm{n}=68)$ by the open-form of StxiB $(p=0.0078)$. Half width of the response was also notably reduced by the mutation $\left(0.3^{8} \pm 0.02 \mathrm{sec}(n=53)\right.$ for control; $0.3 \mathrm{I} \pm 0 . \mathrm{OI}(\mathrm{n}=68)$ for $\mathrm{StxIB}_{\text {of }}$; $p=0.0029)$, while I0-90\% rise time was slightly but not significantly reduced (0.219 \pm 0.009 sec, $\mathrm{n}=53$ for control and $0.197 \pm 0.005, \mathrm{n}=68 ; p=0.1639$ for $\mathrm{StxiB}_{\mathrm{of}}$ ).

These data support our hypothesis that the open-form mutation of StxiB increases the kinetics of hypertonic solution-mediated neurotransmitter release; therefore, it provides direct evidence for a shift in the energetics of $\mathrm{Ca}^{2+}$-independent neurotransmitter release. 


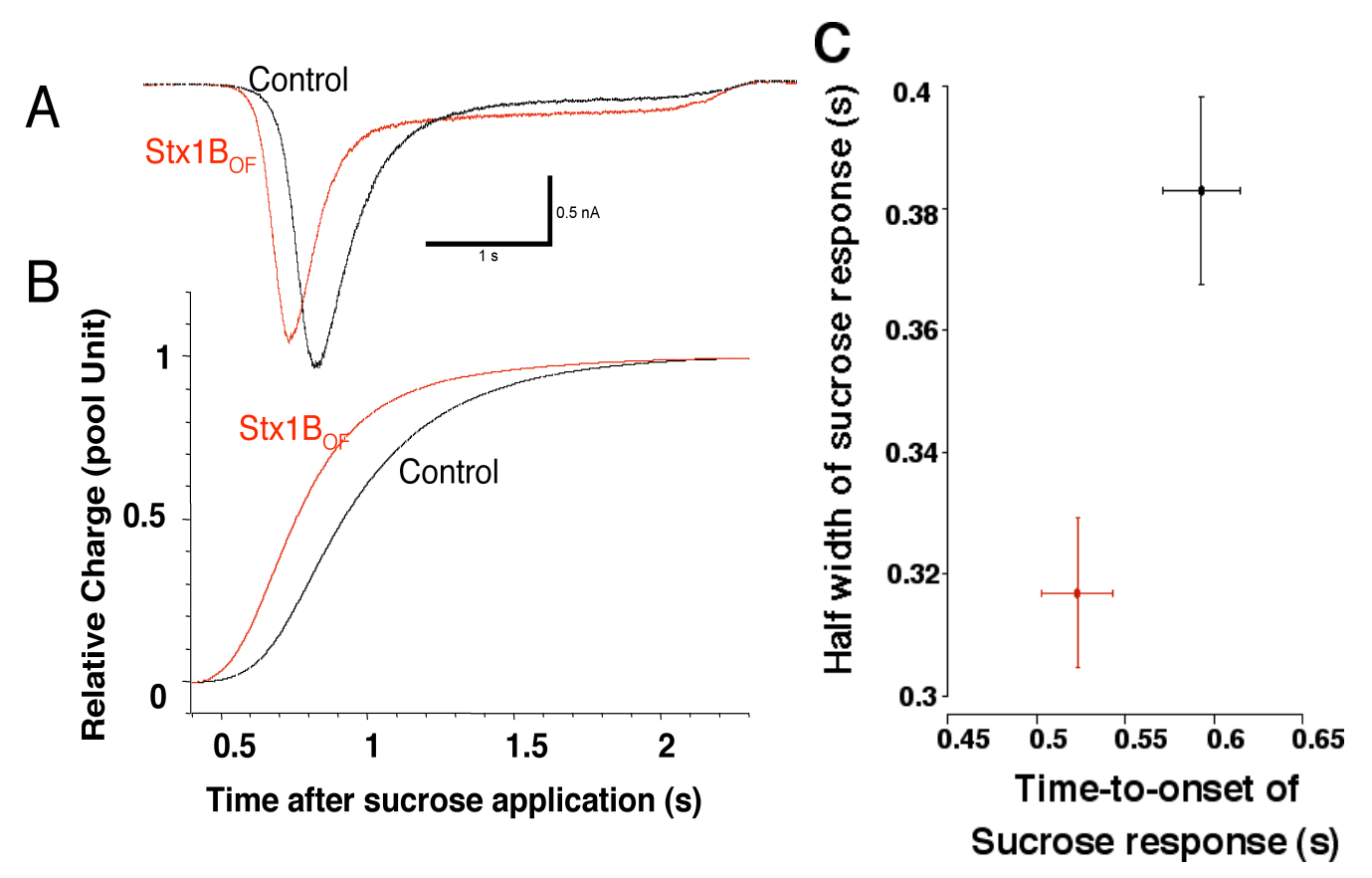

[Figure 19] Time courses of neurotransmitter release by $500 \mathrm{mM}$ sucrose. A, To obtain an average sucrose responses curve in each group. First, the sucrose responses were aligned to time of the peak location to compare average shape, and then shifted according to the average time of onset to elucidate changes in release kinetics. B, The averaged sucrose responses were integrated and normalized to total charge to compare cumulative released charge from the pool. By this comparison, a narrower and a leftward shift in average sucrose response was gained in Stx $1 B_{\text {of }}$ neurons. Cumulative charge vs time curve of Stx $1 \mathrm{~B}_{\text {of }}$ shows an earlier onset as well as a faster time course of release in response to the same osmotic pressure compared to control $(p<0.005)$. C, Half-width and time-to-onset were measured from individual sucrose responses. Responses that deviated more than three times S.D. from average were ruled out. 


\section{I8 Neurotransmitter release by smaller osmotic pressure}

For further confirmation of increased spontaneous efficiency for fusion, we designed a set of follow-up experiments. First, we compared the ratio of transient component charge caused by lower osmotic pressure $(250,350 \mathrm{mM}$ sucrose $)$ to RRP charge for control and $\mathrm{StxI}_{\mathrm{of}}$. Since the transient inward component induced by hypertonic osmotic pressure represents the faster depletion factor than the steady state refilling component, neurons with increased release rate require smaller osmotic pressure for producing the same charge of transient component.

The result depicted in [Figure $20 \mathrm{~B}]$ firmly supports the hypothesis that less energy is required in $\mathrm{StxI}_{\text {of }}$ to release fusion-competent vesicles. While $250 \mathrm{mM}$ sucrose hypertonic solution induced release as much as $20 \pm 3 \%$ of the RRP in control $(n=2 I)$, up to $54 \pm 6 \%$ of RRP was released in StxiB $B_{\text {of }}$ neurons $(n=20$; $p<0.000 \mathrm{I})$. Also, the response evoked by $35 \circ \mathrm{mM}$ sucrose was $5^{2} \pm 4 \%$ of the RRP in control $(n=25)$, as against $79 \pm 6 \%$ of response evoked by $500 \mathrm{mM}$ in $\mathrm{StxiB}_{\text {of }}$ $(\mathrm{n}=22 ; p=0.00 \mathrm{I} 6)$. As expected, the same mechanical force applied to StxiB $\mathrm{B}_{\text {of }}$ containing synapses caused release of a greater fraction of the pool.

However, current transient at particularly lower hypertonicity is hard to detect as the responses reach the steady state without forming a peak. We therefore determined vesicular release rate as a function of hypertonic pressure. Cells were 
subjected to different doses of sucrose hypertonic solution (IOO, 25०, 35 ०, and 500 $\mathrm{mM}$ ) and calculated release rate of responses in each concentration.

For measurement of maximum release rate by $250 \mathrm{mM}, 350 \mathrm{mM}$ and 500 $\mathrm{mM}$ sucrose, we integrated the transient component of each response and normalized by the charge released by $500 \mathrm{mM}$ sucrose hypertonic solution, which termed as RRP for direct comparison across neurons. From this normalized integral, maximum slope was fitted.

For measurement of release rate at Ioo mM sucrose, we used miniature events detection program and the release rates calculated based on the frequency of the events. IOO $\mathrm{mM}$ sucrose was applied for 5 os and the frequency of minis under this condition was monitored. Release rate by Ioo $\mathrm{mM}$ sucrose was calculated from the respective mEPSC rates divided by the corresponding number of vesicles in the RRP of each cell. [Figure $20 \mathrm{C}$ ]. With these methods of analysis in hand, we were able to directly measure the maximum vesicular release rate in pool unit/s.

As expected when $\mathrm{StxiB}_{\text {of }}$ neurons have higher efficiency of $\mathrm{Ca}^{2+}$ independent membrane fusion, we observed the $\mathrm{StxI}_{\text {of }}$ group showed faster vesicular release rate in every concentration of sucrose tested [Figure $20 \mathrm{C}$ ]. Except for the vesicular release rate at IOO $\mathrm{mM}(0.015 \pm 0.002$ pool unit $/ \mathrm{s}, \mathrm{n}=\mathbf{2 2}$ for control and $0.024 \pm 0.003$ pool unit/s, $\mathrm{n}=\mathrm{I} 9$ for $\left.\mathrm{StxiB}_{\text {of }}, p=0.05 \mathrm{I} 5\right), \mathrm{Stxi}_{\text {of }}$ mutation led to enhanced release rate. The maximum release rate at $250 \mathrm{mM}$ sucrose showed o.IO \pm 0.02 pool unit/s $(n=I 7)$ for control, $0.37 \pm 0.07$ for $\operatorname{StxIB}_{\text {of }}(n=I 8 ; p<0.000 I)$. In 
response to $350 \mathrm{mM}$ sucrose, $0.40 \pm 0.04 \mathrm{pool}$ unit/s $(\mathrm{n}=\mathrm{I} 7)$ and $0.90 \pm 0 . \mathrm{IO}$ pool unit/s $(n=18)$ were released $(p=0.0010)$. For $500 \mathrm{mM}$ sucrose, I.24 \pm 0.04 pool unit/s (n=I7) and I.64 \pm o.IO pool unit/s ( $=$ = I7; $p=0.0$ IO9) was observed in control and $\mathrm{StxiB}_{\text {of }}$, respectively.

We also added the spontaneous release rate at $\mathrm{mM}$ hypertonicity into the plot to show that even without any external force, the release rate is enhanced and therefore the behavior of $\mathrm{StxiB}_{\text {of }}$ at rest is consistent with our finding with stimulation of hypertonic solution. As previously described, spontaneous release rates (without hypertonicity) were drastically enhanced by constitutive opening of StxiB (0.00I5 pool unit/s $\pm 0.0004(\mathrm{n}=62)$ in control and $0.0033 \pm 0.0004$ pool unit/s $(n=62)$ in $\left.\mathrm{StxiB}_{\mathrm{of}} ; \quad p<0.000 \mathrm{I}\right)$. Therefore, we can conclude that openmutation of StxiB enhanced $\mathrm{Ca}^{2+}$ independent release rate both in spontaneous release activity as well as in the accelerated kinetics by hypertonic pressure.

In summary, it is likely that, at least in larger part, the observed increase in vesicular release rate during evoked responses in $\mathrm{StxI}_{\text {of }}$ is caused by a reduced energy barrier for $\mathrm{Ca}^{2+}$-independent fusion of the vesicles. 


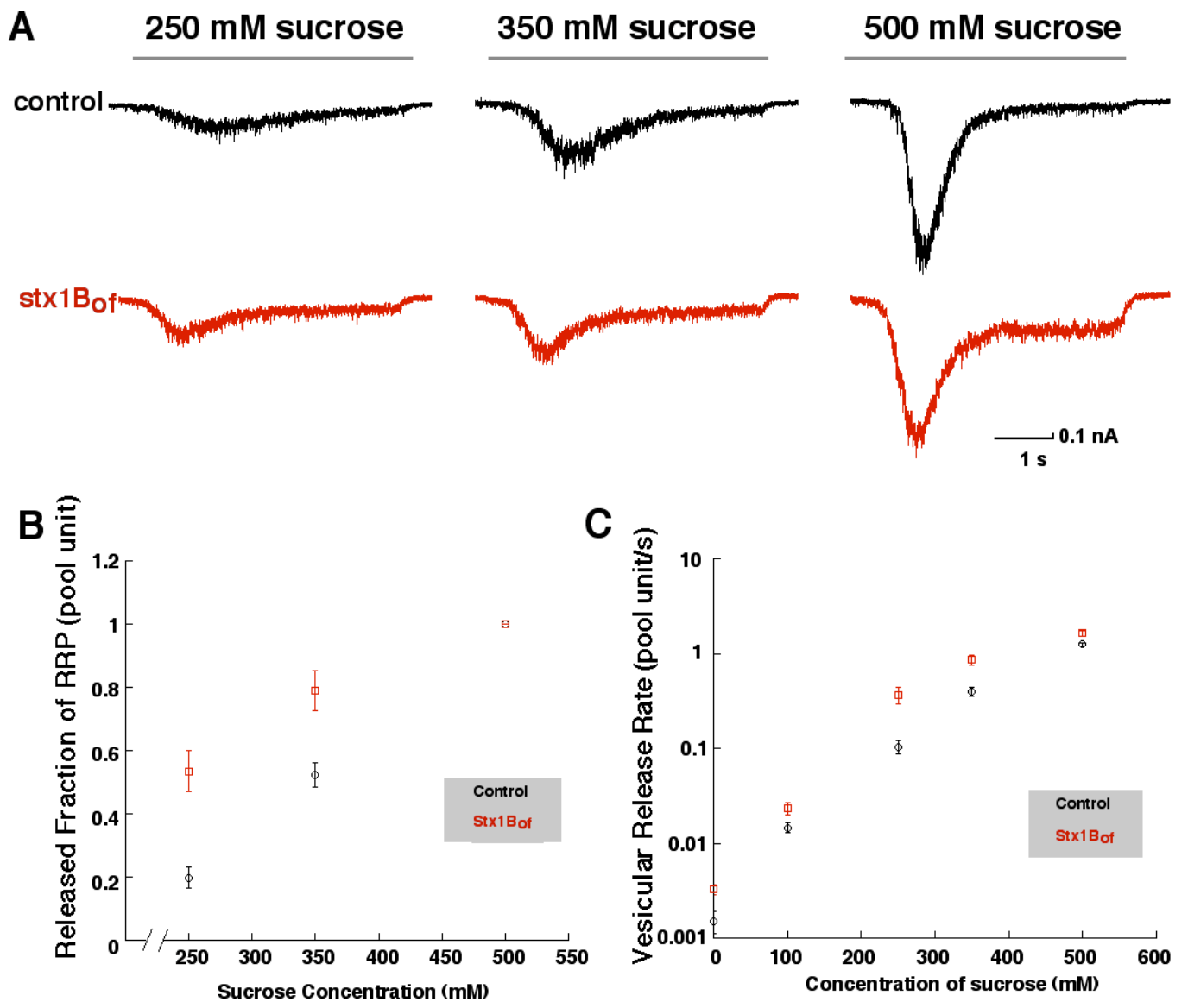

[Figure 20] Determination of an energy barrier for fusion by released fraction of RRP and maximum released pool unit/s. To test the possibility that the open-form mutation leads to a lower energy barrier for fusion osmotic pressure lower than $500 \mathrm{mM}$ sucrose was applied. The relative pool units released and release rate at various concentration of hypertonic solution (0-500 mM) were compared. A, Representative sample traces of responses evoked by 250, 350 and $500 \mathrm{mM}$ hypertonic sucrose solution. Gray bars above traces show time hypertonic solution was applied. B, Relative charge released in response to 250 and $350 \mathrm{mM}$ sucrose to charge of RRP C, Calculated pool unit per second in various concentrations of sucrose. Released pool unit/s at 250, 350 and $500 \mathrm{mM}$ was determined as maximum slope of normalized, integrated transient component. Release rate at 0 and $100 \mathrm{mM}$ were gained by the mini frequencies over total number of vesicles in RRP. 


\section{I9 Priming rate of vesicles}

So far, we have analyzed the effect of open-form mutation on the efficiency of neurotransmitter release. Since switching conformation of Stxi is one of the major events during the priming reaction, one of the initial questions was whether switching conformation of Stxi indeed improves efficiency of priming rate.

As mentioned previously, mean amplitude of the steady state component of the sucrose response conveys information about the refilling rate, thus, priming efficiency of RRP. We have already pointed out that $\mathrm{Stxi}_{\text {of }}$ neurons show larger steady state inward current from the averaged shape of sucrose response (figure I9) compared to StxiAKO control, which behaves like wild-type (figure I2).

Assuming that refilling is a linear process, the mean amplitude divided by the charge of RRP depicts estimated refilling pool unit per second. Absolute currentinduced steady state inward activity was slightly but not significantly larger in StxiB $_{\text {of }}$ cells (Figure $2 \mathrm{IB} ; \mathrm{I} 20 \pm$ IO pA, $\mathrm{n}=66$ for control; $\mathrm{I} 80 \pm 20 \mathrm{pA}, \mathrm{n}=96$ $p=0.0933)$. Taking the overall smaller pool size in $\mathrm{StxIB}_{\text {of }}$ into consideration, the difference was strongly significant. (Figure 2IC; 0.II6 pool unit/s $\pm 0.008 \mathrm{n}=66$ for control and 0.370 pool unit/s $\pm 0.030, \mathrm{n}=96$ for $\left.\mathrm{StxIB}_{\text {of }} ; p<0.000 \mathrm{I}\right)$. 
A more reliable evaluation for rate of pool refilling is to apply pairs of 500 $\mathrm{mM}$ sucrose at varying inter-pulse intervals (I, 4, 7 and Io sec). The first application of sucrose depletes the RRP, while the second sucrose response shows refilled fraction of the RRP during the inter-pulse interval. As can be correlated with the previous result from the measurement of steady state inward current of the sucrose response (figure I9 and figure 2I), refilling kinetics of the pool is notably faster in StxiB $B_{\text {of }}$ group $\left(\tau=2.3255^{\mathrm{S}}\right.$ in control: $\tau=\mathrm{I} .082 \mathrm{~S}$ in $\mathrm{StxIB}_{\text {of }}$; figure 22). For example, one second after pool depletion, RRP of $\mathrm{StxI}_{\text {of }}$ was refilled as much as $0.50 \pm 0.04$ of the total pool $(n=18)$, while $0.30 \pm 0.02$ pool unit $(n=I 8)$ was replenished in the control state $(p=0.0003)$.

These results concerning the rate of RRP refilling imply that the opening of Stxi may be one of time limiting steps in the priming reaction.

\subsection{Diacylglycerol/ $\beta$-phorbol ester induced augmentation}

According to current hypotheses, opening of StXI is mediated by essential priming factor, Munci3 (Augustin et al., I999; Richmond et al., 200I). If the only role of Munciz in exocytosis is to open Stxi, its role of Munciz in the $\mathrm{StxiB}_{\text {of }}$ neuron, should have become concealed. Besides being an essential priming factor, Muncizs are also the sole target of diacylglycerol (DAG)/ $\beta$-phorbol ester $(\beta$-PE) mediating augmentation of neurotransmitter release (Rhee et al., 2002). We used this property 
to test whether Munciz is still acting on the priming/release process. If $\beta$-PE still causes potentiation of the synaptic response in $\mathrm{StxI}_{\text {of }}$ neurons, this would indicate that Munciz does more than opening Stxi. In contrast, if Munciz only opens StXI as its sole role in regulation of exocytosis, $\beta$-PE should not induce any change in synaptic responsiveness in $\mathrm{StxI}_{\text {of }}$ neurons.

We, therefore, measured potentiation of synaptic responses upon application of I $\mu \mathrm{M} \beta$-phorbolester dibutyrate (PDBU). After obtaining base line responses I $\mu \mathrm{M}$ PDBU was applied for I min and was subsequently washed out for 5 min. Amplitudes of EPSCs in PDBU were normalized to the initial base line, and the degree of potentiation was measured by EPSCs after augmented responses reach steady state. While control cells show $2.44 \pm 0.13$ fold enhanced neurotransmitter release $(n=45), \mathrm{StxIB}_{\text {of }}$ cells show much reduced enhancement of I.45 \pm 0.05 fold by $\operatorname{PDBU}(n=43, p<0.000 \mathrm{I}$; figure $23 \mathrm{~A})$. This data, depicting that $\mathrm{StxIB}_{\text {of }}$ still shows sensitivity in response to PDBU, leads to the conclusion that apart from opening Stxi, Munciz indeed plays additional roles in the synaptic release process.

Furthermore, the reduced PDBU response could be the consequence of the intrinsic high release probability of $\mathrm{StxIB}_{\text {of }}$, while the EPSC of $\mathrm{StxIB}_{\text {of }}$ is as sensitive as control to PDBU, implying that although Munci3 may exercise part of its function by switching the conformation of StxI, PDBU-stimulated Munc I3 could employ a different mechanism to enhance neurotransmitter release.

To evaluate this possibility we correlated the degree of augmentation of EPSC as a function of vesicular release probability. If high vesicular release 
probability was the cause of the reduction in PDBU augmentation, we would see inverse correlation between the two parameters.

Figure $23 \mathrm{~B}$ shows the combination of vesicular release probability and PDBU augmentation measured from both control and $\mathrm{Stxi}_{\text {of }}$ neurons. Stxi $\mathrm{B}_{\text {of }}$ (red dots) have intrinsically higher vesicular release probability and smaller degree of potentiation in comparison to control (black dots; figure ${ }_{23} \mathrm{C}$ ). However, a continuously distributed inverse correlation appears to form between these two parameters. Based on this finding, we would propose that the reduction in degree of potentiation by $\mathrm{PDBU}$ in $\mathrm{StxI}_{\text {of }}$ is due to intrinsically high vesicular release probability rather than impaired interaction of Munciz with its target in changing neurotransmitter release probability.

However, in all cases, the augmentation of neurotransmitter release in $\mathrm{StxiB}_{\text {of }}$ neurons suggest that Munc I3-I has a role alternative to that of switching the conformation of Stxi in response to PDBU and thus to DAG. 


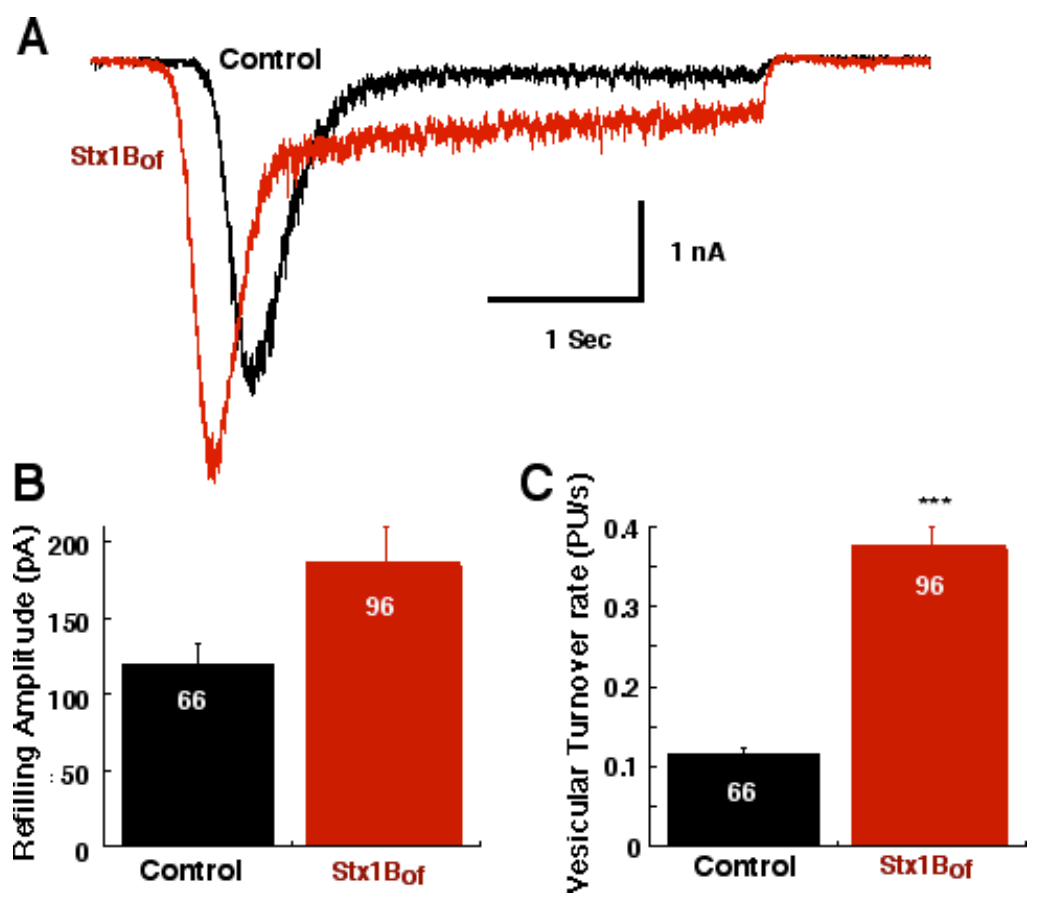

[Figure 21] Steady state amplitude of sucrose response. Sustained component of sucrose response was termed refilling amplitude. A, Representative traces (Black-control; red-Stx $1 \mathrm{~B}_{\mathrm{of}}$ ) $\mathbf{B}$, Average of mean amplitude in sustained component $\mathbf{C}$, Mean amplitude of steady state component was divided by the charge of RRP to standardize various pool size (refilling pool unit/s). Refilling of RRP in Stx1B of cells were significantly faster than control. ${ }^{* * *} p<0.0001$ 

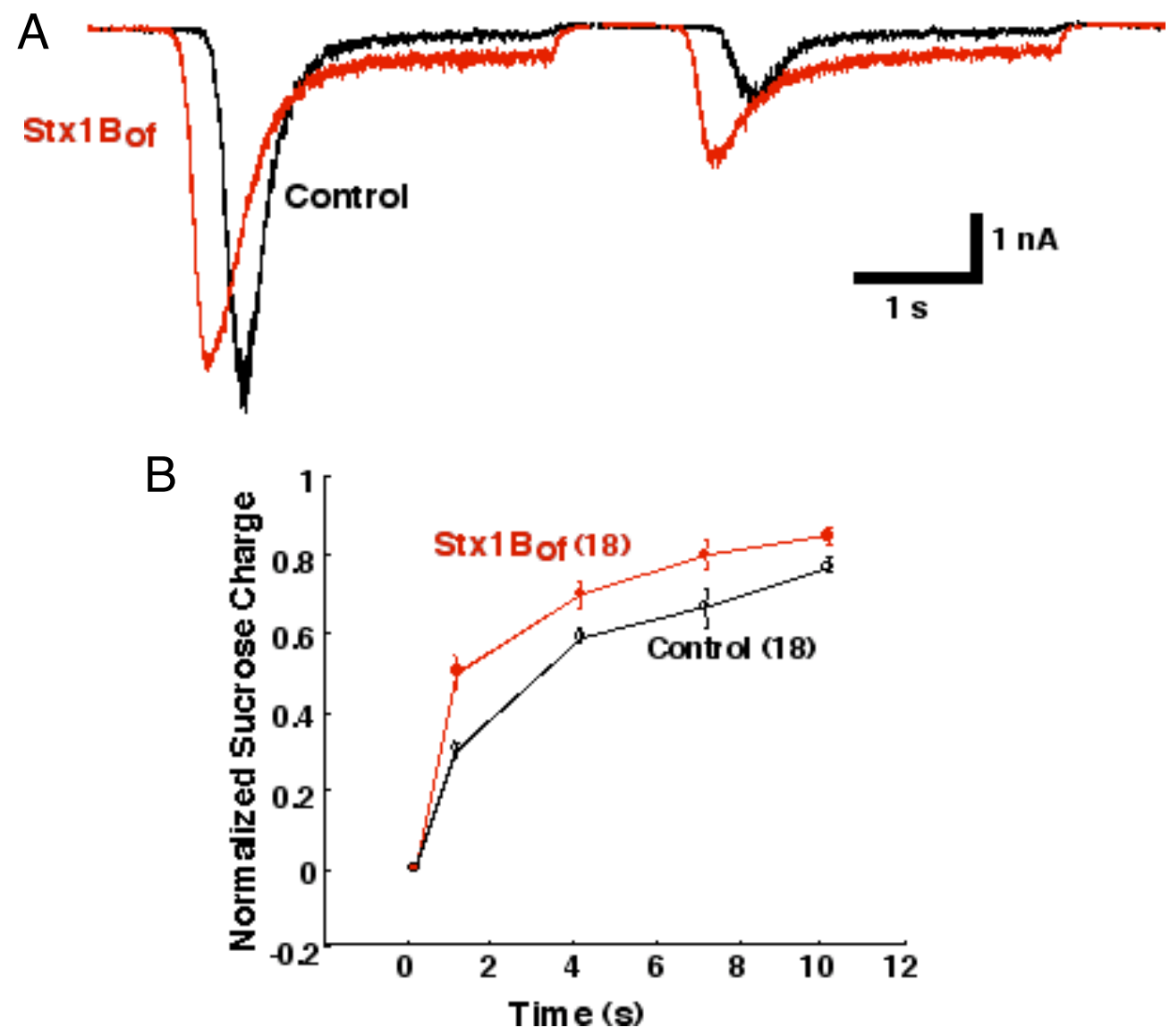

[Figure 22] Comparison of priming rate between control and Stx1B $\mathrm{B}_{\text {of }}$ neurons. To compare priming rate of vesicles into the RRP, paired sucrose responses were compared. After a certain time from depletion of the pool by first sucrose application, sucrose was re-applied and the ratio for refilled charge compared to the first is determined as refilling of the time internal. A, Sample traces for each group (Blackcontrol; Red-Stx $\left.1 \mathrm{~B}_{\mathrm{of}}\right)$. B, After $1 \mathrm{~s}$ of depletion around half of RRP was refilled in Stx $1 \mathrm{~B}_{\text {of }}$ cell, while only $30 \%$ of the pool is refilled in control. This difference correlates well with the finding from the sustained component of sucrose response. It should be noted from the identical offset points of the sucrose responses in the control and Stx $1 B_{\text {of }}$ raw traces that the faster onset is due to inherent release properties of Stx $1 B_{\text {of }}$ and not the fast flow application system. 
A

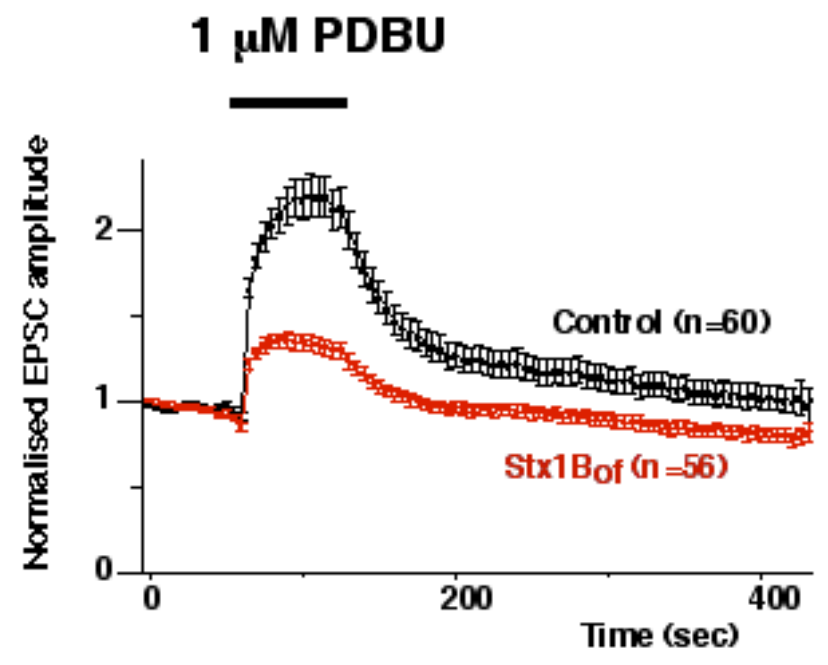

B

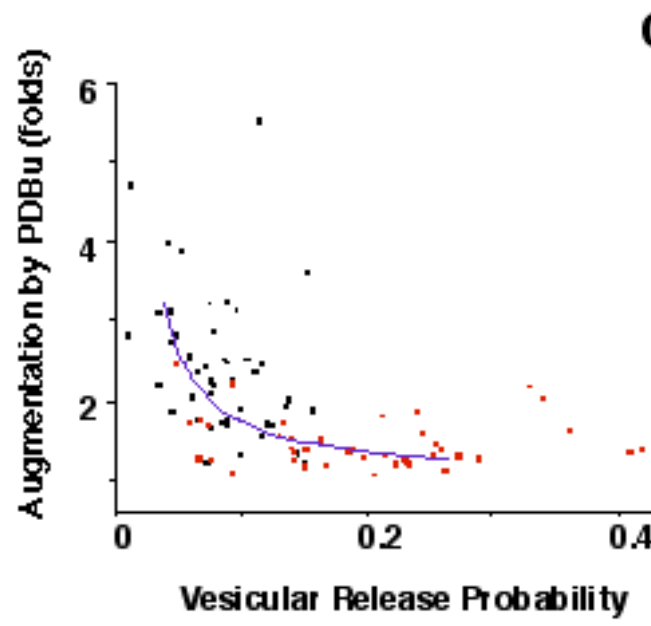

C

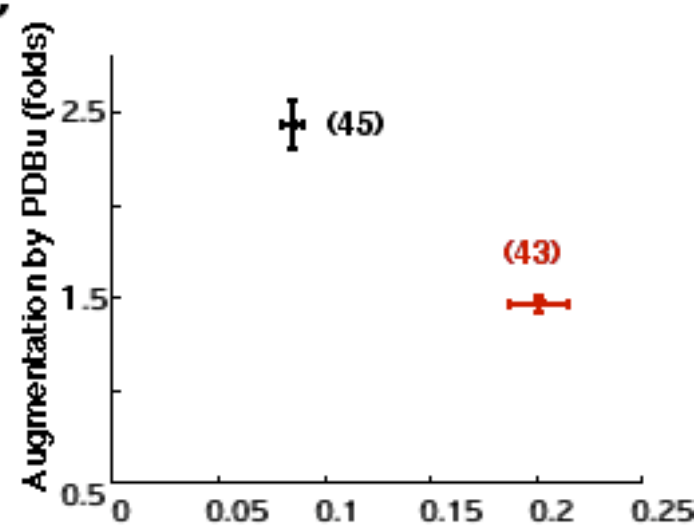

Vesicular Release Probability

[Figure 23] Alternative role of Munc13 in vesicle priming/fusion. A, To examine whether Munc13-1 has different role than switching conformation of Stx1, the level of augmentation by PDBU was measured. While control neurons show $2.44 \pm 0.13$ fold enhanced EPSCs, Stx $1 \mathrm{~B}_{\text {of }}$ neurons have $1.45 \pm 0.08$ fold augmentation in response to PDBU. B. To resolve whether reduced degree of potentiation in Stx $1 \mathrm{~B}_{\text {of }}$ is due to intrinsically high release probability, the degree of augmentation was plotted as a function of release probability (Black-control; Red-Stx $1 \mathrm{~B}_{\mathrm{of}}$ ). Note that two groups fall in a continuous distribution forming inverse correlation between these two parameters. C. Mean release probability and potentiation are plotted. Error bar shows S.E.M. Numbers in blanket shows number of cells tested. 
5. Discussion 


\section{DISCUSSION}

The purpose of this study was, first, to identify functional differences between StxiA and StxiB and secondly, to study the physiological impact of mutation of SNARE molecule, Stxi that has been found in biochemical experiment to lock the conformation of Stxi in open form and prevent the formation of closed conformation.

We initially characterized synaptic properties of mice lacking StxiA and found that synapses lacking StxiA do not lead to any obvious defects in neurotransmission in comparison to wild-type synapses. Subsequently, StxiAKO mice were crossed with $\mathrm{StxI}_{\text {of }}$ mutant mice to obtain homogeneous conformation of Stxi to direct compare between StxiB wild-type and StxiB $_{\text {of }}$. Mice expressing an open mutation of StxiB on a StxiA knock out background exhibited a 2.2 fold increase in release efficacy most likely caused by a reduction in the energy barrier for membrane fusion. In addition, synapses containing the constitutively open conformation of StxiB led to significantly faster vesicle priming.

Despite lines of evidence predicting functional differences between the two major secretory cell-specific isoforms of syntaxin, namely, StxiA and StxiB, including differential distribution, binding affinity to Muncis and inhibitory roles on 
voltage-gated $\mathrm{Ca}^{2+}$ channel (see introduction part 2.9), StxiA null mice showed typical hippocampal synaptic release properties of wild-type mice.

A possible explanation for this finding might be the presence of StxiB as the dominant endogenously expressed isoform in the hippocampal neurons was considered. However, in keeping with previous studies, our immunohistochemistry result shown in the present study indicates that these two isoforms are natively expressed throughout the hippocampal area (see figure 8 and (Ruiz-Montasell et al., 1996)). Therefore StxiB cannot be the predominant isoform in hippocampus.

In general, deletion of one isoform may lead to an enhanced expression of the other. However, the lack of StxiA does not detectably affect the expression of StxiB. This can be explained by the presence of alternative isoforms of syntaxin other than StxiB, as a compensatory measure for the absence of StxiA. Alternatively and more likely, StxiA may not functionally differ from StxiB and there can be substantially great amounts of total Stxi present in hippocampal neurons as has been shown previously by Hu et al. (Hu et al., 2002).

To provide a clearer answer for the functional differences between the two isoforms, we would need to compare the synapses using only StxiA and StxiB, respectively. The comparison between neurons from anterior lobes of pituitary gland where no StxiB was found (Ruiz-Montasell et al., I996) and that of StxiAKO 
mice may provide a better resolution. Furthermore, the parallel analysis of StxiB null mice, or characterization of the double knock out synapses rescued by either of those isoforms will be needed.

As previously described in the introduction (part 2.9), Stxi has at least two conformations: a closed conformation in which core complex domain is masked by the N-terminal Habc domain, so that it is unable to form SNARE complexes, and an open conformation, where the SNARE motif of StxI is exposed and ready to form SNARE complexes, possibly through an intermediate conformation. However, it has not yet been verified what can be caused from the open conformation of StxI.

Since a conformational switch to the open state is believed to be a key step in the priming reaction, an enhanced priming rate in $\mathrm{StxI}_{\text {of }}($ Figure 2I, 22) is not too surprising. Vesicles docked at active zones in $\mathrm{StxIB}_{\text {of }}$ most likely have a higher probability to face an active Stxi (open-conformation of Stxi), without having to wait for being acted upon by the priming proteins. We also discovered that the vesicles primed with StxiB that are constitutively open have higher fusion efficiency than the vesicles with wild-type StxiB. Since even in the wild-type context, all the primed vesicles are, in any case, supposed to have open Stxi, this observation was rather unforeseen. 
The increased vesicular release probability was determined by two independent measurements. The bigger population of vesicles was released by an action potential and secondly the short-term plasticity experiment revealed much stronger depression, which is an indicative of higher release probability.

Nevertheless, we observed that the total synaptic output per action potential per synapse was identical between the StxiAKO control and the open-form mutant. This was demonstrated by similar amplitudes of EPSC (Figure 13) as well as by unchanged synaptic release probability, measured using the MK-80I method (Figure I5). Based on these findings, together with the same size of EPSC, we could conclude that the increased vesicular release probability and decreased size of RRP compensates each other to hold the same synaptic strength as in wild-type neurons.

These two findings correlate well with decreased RRP size in $\mathrm{StxiB}_{\text {of }}$ neurons (Figure 13). Reduced RRP size was also rather surprising because we noticed that the vesicle priming rate is actually enhanced. If the equilibrium between the priming and umpriming is relevant for size of the RRP, we would expect the opposite, an increase in pool size. Thus, we suggest that the reduced RRP size is a compensatory mechanism to preserve the size of EPSC in the effective range so as to maintain consistency of synaptic transmission. 
The cause of increased release efficacy can be explained by reduced activation energy of the fusion reaction, based on the following findings: (I) increased level of vesicular release probability coincides with that of spontaneous release probability [figure 13 and $\mathrm{I} 8$ ]. (2) early onset, and narrower half width of the sucrose responses indicating faster kinetics and increased efficiency of vesicular fusion by given constant osmotic pressure [figure 19]. (3) a larger fraction of the pool was released at lower concentration of sucrose, implying ease of fusion [figure $20 \mathrm{~B}$ ] (4) increased release rate (higher pool unit/s) by every given concentration of sucrose [figure $20 \mathrm{C}]$. Similar phenomena were observed in the previous studies by $\beta$-PE stimulation (Stevens and Sullivan, 1998) and by high frequency stimulation (Stevens and Wesseling, 1999)

One of the possible explanations would be following. Stxi continuously switches its conformation with binding to either Muncr3 or Munc I8-I, reaching equilibrium between the open and closed state. The greater the portion of the core complex region exposed, the more completely the SNARE complex forms. Hence, a greater part of the unstable hemifusion intermediate is stabilized energetically. In the open form mutant, because the equilibrium is set to the fully open state, every SNARE formed is a complete one. Thus, intermediate states are stabilized to a greater extent, which drives the fusion process more easily. However, a half-formed SNARE has not been reported so far in the literature and it is not known whether Muncr3 binding is reversible, hence, this idea cannot be evidently theorized. 
Another possible explanation for the increased release can be suggested based on an observed obvious left shift in sensitivity of release machinery to external $\mathrm{Ca}^{2+}$ concentration as a cause of phenotype. Elevation of external $\mathrm{Ca}^{2+}$ in $\mathrm{StxiB}_{\text {of }}$ caused reduced potentiation and the synapses using $\mathrm{StxI}_{\text {of }}$ have less sensitivity to reduction of external $\mathrm{Ca}^{2+}$, which is consistent with higher sensitivity to $\mathrm{Ca}^{2+}$.

As explained above, Stxi has a negative regulatory effect on $\mathrm{Ca}^{2+}$ channels. If introduction of the mutation in Stxi molecule causes des-inhibition of $\mathrm{Ca}^{2+}$ channel function, then it will subsequently lead to a greater $\mathrm{Ca}^{2+}$ influx and enhanced sensitivity to external $\mathrm{Ca}^{2+}$. The possibility of altered regulation of $\mathrm{Ca}^{2+}$ channels is supported by the evidence that transiently transfected tsA-2or cells with an open conformation of Stxi did not show a hyperpolarizing shift in the half-inactivation potential of N-type $\mathrm{Ca}^{2+}$ channel (Jarvis et al., 2002).

Since the preparation we used in this study does not allow direct measurement of $\mathrm{Ca}^{2+}$ current at the presynaptic terminal, we measured somatic $\mathrm{Ca}^{2+}$ current to verify this possibility. Furthermore, for providing a better resolution, the L-type-dependent $\mathrm{Ca}^{2+}$ current, which normally contributes little in neurotransmission, was blocked using nifedipine and the amplitude of the P/Q- and $\mathrm{N}$ - type channel-dependent current was compared. We found that both in the presence and absence of the L-type $\mathrm{Ca}^{2+}$ channel contribution, the somatic current remained unchanged (Figure I7). In spite of this finding, the possible influence of 
Stxi open mutation on $\mathrm{Ca}^{2+}$ channel inhibition cannot be completely ruled out, since one has to consider the fact that reliable electrical control of the presynaptic $\mathrm{Ca}^{2+}$ current is hard to achieve. The membrane of neuronal processes is normally to some extent isolated from the electrode leading to separation by an axial access resistancedepending on the distance between the membrane and the electrode. This introduces significant voltage-drop or space clamp error, leading to inaccuracy in estimation of actual presynaptic $\mathrm{Ca}^{2+}$ current. Therefore, further studies involving direct measurement of the presynaptic $\mathrm{Ca}^{2+}$ current, such as Calyx of Held (Felmy et al., 2003), will be needed to overcome this problem.

In the light of these ideas, undetected difference in $\mathrm{Ca}^{2+}$ influx at the presynaptic terminal could indeed explain the increased release probability, and enhanced priming rate, but not the parallel observations of increased rate of spontaneous release or the kinetic change of sucrose response. In addition, based on the finding that the level of increased spontaneous release probability correlates with increased vesicular release probability, differential regulation of $\mathrm{Ca}^{2+}$ channels cannot be the major cause.

The most reasonable explanation would be that in $\mathrm{StxiB}_{\text {of }}$, there are increased numbers of SNARE complexes per vesicle and this causes the observed phenotype. Under physiological conditions, free Stxi switches its conformation to establish equilibrium between the open and closed conformations in a short time 
(Margittai et al., 2003b) and the open-form mutation shifts the equilibrium towards the open state.

In order to sustain the open, active conformation, Stxi needs extra interaction with regulatory proteins such as Munc I3 and RIM (Koushika et al., 200Ib; Richmond et al., 200I). This requirement for interactions decrease the chances of having open-form Stxi in comparison to the $\mathrm{StxIB}_{\text {of }}$ situation, where all of the Stxi molecules are already in their open and active state. The number of SNARE complexes critically depends on the proportion of open-form StxI. The more open-form of StxI, the larger number of assembled SNARE complexes are formed between the plasma and vesicle membrane. Accordingly, more SNARE complexes could be formed per vesicle, and each of the complexes may provide the energy needed to drive fusion (Lin and Scheller, I997). Such vesicles with inherently open form of Stxi need to overcome lower threshold energy for fusion and therefore they fuse with noticeably higher release probability and accelerated fusion kinetics.

The results presented so far have shown that opening of Stxi, known to be a functional consequence of interaction with Munci3-I, makes vesicles more ready for priming, as well as for fusion. This raises the question whether enhancing the function of Munci3-I in wild-type neurons can lead to similar phenomena i.e. enhanced release probability. Recent unpublished data from our laboratory, as well as a previous report show that PDBU application causes similar phenomena with open mutation of Stxi, in terms of earlier onset of sucrose response, and faster pool 
unit depletion by sucrose (J.Basu and C. Rosenmund, personal communication (Stevens and Sullivan, 1998)). Based on the finding that Munci3 is the sole molecular target for $\beta$-PE mediated augmentation of neurotransmitter release (Rhee et al., 2002), we can conclude that such a functional enhancement of Munci3 indeed showed similar release properties to those of $\mathrm{Stxi}_{\text {of }}$, which, in turn, support the hypothesis that Muncrz-I is the molecule switching the conformation of StxI.

Now, focus can be directed to answer the question whether conformational switching Stxi is the only role of Munciz. This issue can be addressed by exploring whether Muncr3 can still exert its function for neurotransmitter release in the neuron expressing open-form of Stxi. If the role of Munciz in neurotransmitter release was nothing more than that of opening up StxI, then the effect of PDBU upon Muncrz would not persist in this mutation, and subsequently, augmentation of neurotransmitter release would not be expected. By applying PDBU onto StxiB ${ }_{\text {of }}$ cells, Munciz was stimulated and potentiation of EPSC was observed. Although the level of PDBU-mediated augmentation was significantly reduced in $\mathrm{StxiB}_{\text {of }}$ as compared to its control counterpart, enhanced neurotransmitter release was observed (Figure23).

One possible interpretation of this result is that there is another molecule (or other molecules) that can mediate PDBU-induced augmentation, recruiting a mechanism(s) alternative to that of Munciz. However, this is very unlikely because 
mutation of Munc $\mathrm{I}_{3}$ for lacking phorbol ester interaction $\left(\mathrm{H}_{5} 67 \mathrm{~K}\right.$ mutation) led to completely abolished augmentation under the same experimental condition (Rhee et al., 2002).

Alternatively, considering that PDBU is indeed acting upon Munciz only, and that level of augmentation in the $\mathrm{StxI}_{\text {of }}$ is reduced, PDBU mediated Munci3 stimulation exerts its effect on neurotransmitter release via a different mechanism, independent of Stxi opening. In this case, Munciz does not open Stxi to potentiate the release but acts upon a different target in the fusion process for facilitating the release at least in response to PDBU. Therefore, $\mathrm{StxiB}_{\text {of }}$ cells would show the same level of augmentation with that of wild-type. However, as emphasized by our results, PDBU-mediated augmentation is significantly reduced. This may be because the intrinsically higher, and more saturated vesicular release probability, as seen in the $\mathrm{StxiB}_{\text {of }}$ neurons, may not allow the same degree of PDBU augmentation. Alternatively, it can be also explained if Muncr3 exerts its role in potentiate EPSC, partially by opening StxI, in addition to alternative pathway.

To check this possibility, the augmentation level was plotted against vesicular release probability of the cell (figure $23 \mathrm{~B}, \mathrm{C}$ ). If the assumption is correct, overlapped negative correlation between the two factors is expected. In this study, as plotted in figure $23 \mathrm{~B}$, these two parameters showed relatively weak but overlaid negative correlation between each other. Therefore, we would suggest that the 
reduced augmentation by PDBU is rather the consequence of intrinsically higher release probability.

However, our results so far do not clearly resolve whether the role of Muncr3 in response to $\beta$-PE is to mediate, solely a mechanism that is alternative to opening of StxI or both the opening of StxI in addition to the other mechanism. To provide an answer of this question, further study addressing $\beta$-PE response in $\mathrm{Stxi}_{\text {of }}$ neurons, with artificially lowered release probability equivalent to that of control e.g. by modifying external $\mathrm{Ca}^{2+}$ concentration, is required.

The hypothesis that Munci3 has a different way of enhancing neurotransmitter release, other than opening Stxi conflicts with the finding in $C$. elegans, demonstrating that overexpression of constitutively open form of StxI bypasses the need of uncr3 (Richmond et al., 200I). Recently, the same overexpression experiment has been performed in a mouse system. An open mutation of Stxi overexpressed in Munciz double knockout mouse hippocampal neuron culture showed partial rescue of neurotransmitter release. Although EPSCs were found in this system, these releases showed very rapid run-down, suggesting that the rescue is far from being complete (Rhee and Rosenmund, unpublished data). This finding provides another line of evidence suggesting additional role of Muncrz besides opening of Stxı. This difference between nematode and mammalian data can be explained by the redundancy and complexity of Munciz function in the mammalian system. However, this preliminary data requires very careful 
interpretation, since overexpression of wild-type Stxi can cause $\mathrm{Ca}^{2+}$ channel inhibition and defects in transmission (Bezprozvanny et al., 2000; Stanley, 2003; Zamponi, 2003). Therefore, tightly controlled expression of $\mathrm{Stxi}_{\text {of }}$, ideally under endogenous gene expression, is required to test this hypothesis.

When taken together, our present study suggests that, the individual conformations of Stxi control the probability of formation of the SNARE complex, and thereby act as crucial regulators of $\mathrm{Ca}^{2+}$-independent membrane fusion efficacy and important determinants of short-term plasticity of synaptic transmission. 
6. References 


\section{REFERENCES}

Aguado, F., Majo, G., Ruiz-Montasell, B., Llorens, J., Marsal, J., and Blasi, J. (I999). Syntaxin IA and IB display distinct distribution patterns in the rat peripheral nervous system. Neuroscience $88,437-446$.

Aravamudan, B., Fergestad, T., Davis, W. S., Rodesch, C. K., and Broadie, K. (I999). Drosophila UNC-I3 is essential for synaptic transmission. Nature Neuroscience 2, 965-97I.

Augustin, I., Rosenmund, C., Südhof, T. C., and Brose, N. (I999). Munciz-I is essential for fusion competence of glutamatergic synaptic vesicles. Nature $400,457-46 \mathrm{I}$.

Bekkers, J. M., and Stevens, C. F. (I99I). Excitatory and inhibitory autaptic currents in isolated hippocampal neurons maintained in cell culture. Proc Natl Acad Sci U S A 88, 7834-7838.

Bennett, M. K., Calakos, N., and Scheller, R. H. (I992). Syntaxin: a synaptic protein implicated in docking of synaptic vesicles at presynaptic active zones. Science $257,255^{-2} 59$.

Bennett, M. K., Garcia-Arraras, J. E., Elferink, L. A., Peterson, K., Fleming, A. M., Hazuka, C. D., and Scheller, R. H. (1993). The syntaxin family of vesicular transport receptors. Cell 74, 863-873. 
Betz, A., Okamoto, M., Benseler, F., and Brose, N. (1997). Direct interaction of the rat unc-13 homologue Munci3-I with the $\mathrm{N}$ terminus of syntaxin. Journal of Biological Chemistry 272, 2520-2526.

Betz, A., Thakur, P., Junge, H. J., Ashery, U., Rhee, J. S., Scheuss, V., Rosenmund, C., Rettig, J., and Brose, N. (200I). Functional interaction of the active zone proteins Muncr3-I and RIMI in synaptic vesicle priming. Neuron 30, 183-196.

Bezprozvanny, I., Scheller, R. H., and Tsien, R. W. (1995). Functional impact of syntaxin on gating of N-type and Q-type calcium channels. Nature 378 , $623-626$.

Bezprozvanny, I., Zhong, P., Scheller, R. H., and Tsien, R. W. (2000). Molecular determinants of the functional interaction between syntaxin and $\mathrm{N}$-type $\mathrm{Ca} 2+$ channel gating. Proceedings of the National Academy of Sciences of the United States of America 97, 13943-13948.

Binz, T., Blasi, J., Yamasaki, S., Baumeister, A., Link, E., Südhof, T. C., Jahn, R., and Niemann, H. (1994). Proteolysis of SNAP-25 by types E and A botulinal neurotoxins. Journal of Biological Chemistry 269, I6I7-1620.

Blasi, J., Chapman, E. R., Yamasaki, S., Binz, T., Niemann, H., and Jahn, R. (1993). Botulinum neurotoxin Ci blocks neurotransmitter release by means of cleaving HPC-I/syntaxin. EMBO Journal I2, 482I-4828.

Brenner, S. (1974). The genetics of Caenorhabditis elegans. Genetics 77, 71-94. 
Broadie, K., Prokop, A., Bellen, H. J., O'Kane, C. J., Schulze, K. L., and Sweeney, S. T. (1995). Syntaxin and synaptobrevin function downstream of vesicle docking in Drosophila. Neuron ${ }_{5}, 663-673$.

Brose, N., Hofmann, K., Hata, Y., and Südhof, T. C. (I995). Mammalian homologues of Caenorhabditis elegans unc-I3 gene define novel family of C2-domain proteins. J Biol Chem 270, 25273-25280.

Brose, N., Petrenko, A. G., Südhof, T. C., and Jahn, R. (I992). Synaptotagmin: a calcium sensor on the synaptic vesicle surface. Science 256, IO2I-IO25.

Catterall, W. A. (I999). Interactions of presynaptic Ca2+ channels and snare proteins in neurotransmitter release. Ann N Y Acad Sci 868, I44-I59.

Clements, J. D., Lester, R. A., Tong, G., Jahr, C. E., and Westbrook, G. L. (I992). The time course of glutamate in the synaptic cleft. Science 258 , I498-I5OI.

Deitcher, D. L., Ueda, A., Stewart, B. A., Burgess, R. W., Kidokoro, Y., and Schwarz, T. L. (1998). Distinct requirements for evoked and spontaneous release of neurotransmitter are revealed by mutations in the Drosophila gene neuronal-synaptobrevin. J Neurosci $I 8,2028-2039$.

Dick, O., tom Dieck, S., Altrock, W. D., Ammermuller, J., Weiler, R., Garner, C. C., Gundelfinger, E. D., and Brandstatter, J. H. (2003). The presynaptic active zone protein bassoon is essential for photoreceptor ribbon synapse formation in the retina. Neuron $37,775-786$.

Dobrunz, L. E., and Stevens, C. F. (1997). Heterogeneity of release probability, facilitation, and depletion at central synapses. Neuron I8, 995-Io08. 
Dulubova, I., Sugita, S., Hill, S., Hosaka, M., Fernandez, I., Südhof, T., and Rizo, J. (I999). A conformational switch in syntaxin during exocytosis: role of Munci8. The EMBO Journal $I 8,4372-4382$.

Fasshauer, D. (2003). Structural insights into the SNARE mechanism. Biochim Biophys Acta I64I, 87-97.

Felmy, F., Neher, E., and Schneggenburger, R. (2003). The timing of phasic transmitter release is Caz+-dependent and lacks a direct influence of presynaptic membrane potential. Proc Natl Acad Sci U S A IOO, I5200$\mathrm{I} 52 \mathrm{O}$.

Fernandez, I., Ubach, J., Dulubova, I., Zhang, X., Südhof, T. C., and Rizo, J. (1998). Three-dimensional structure of an evolutionarily conserved Nterminal domain of syntaxin IA. Cell 94, 84I-849.

Fernandez-Chacon, R., Konigstorfer, A., Gerber, S. H., Garcia, J., Matos, M. F., Stevens, C. F., Brose, N., Rizo, J., Rosenmund, C., and Südhof, T. C. (200I). Synaptotagmin I functions as a calcium regulator of release probability. Nature $4 \mathrm{IO}, 4 \mathrm{I}-49$.

Geppert, M., Goda, Y., Hammer, R. E., Li, C., Rosahl, T. W., Stevens, C. F., and Südhof, T. C. (I994). Synaptotagmin I: a major Caz+ sensor for transmitter release at a central synapse. Cell 79, 717-727.

Harlow, M. L., Ress, D., Stoschek, A., Marshall, R. M., and McMahan, U. J. (200I). The architecture of active zone material at the frog's neuromuscular junction. Nature 409, 479-484. 
Hata, Y., Slaughter, C. A., and Südhof, T. C. (I993). Synaptic vesicle fusion complex contains unc-I 8 homologue bound to syntaxin. Nature $366,347^{-}$ 35 I.

Helme-Guizon, A., Davis, S., Israel, M., Lesbats, B., Mallet, J., Laroche, S., and Hicks, A. (1998). Increase in syntaxin IB and glutamate release in mossy fibre terminals following induction of LTP in the dentate gyrus: a candidate molecular mechanism underlying transsynaptic plasticity. European Journal of Neuroscience IO, 223I-2237.

Hu, K., Carroll, J., Fedorovich, S., Rickman, C., Sukhodub, A., and Davletov, B. (2002). Vesicular restriction of synaptobrevin suggests a role for calcium in membrane fusion. Nature $4 I_{5}, 646-650$.

Jagadish, M. N., Tellam, J. T., Macaulay, S. L., Gough, K. H., James, D. E., and Ward, C. W. (I997). Novel isoform of syntaxin I is expressed in mammalian cells. Biochemical Journal 32I, I5 $5^{\mathrm{I}-\mathrm{I}} 5^{6}$.

Jahn, R., Lang, T., and Südhof, T. C. (2003). Membrane fusion. Cell II2, 5I9-533.

Jarvis, S. E., Barr, W., Feng, Z. P., Hamid, J., and Zamponi, G. W. (2002). Molecular determinants of syntaxin I modulation of N-type calcium channels. Journal of Biological Chemistry 277, 44399-44407.

Jarvis, S. E., Magga, J. M., Beedle, A. M., Braun, J. E., and Zamponi, G. W. (2000). G protein modulation of $\mathrm{N}$-type calcium channels is facilitated by physical interactions between syntaxin IA and Gbetagamma. Journal of Biological Chemistry 275, 6388-6394. 
Jarvis, S. E., and Zamponi, G. W. (200I). Distinct molecular determinants govern syntaxin IA-mediated inactivation and G-protein inhibition of N-type calcium channels. Journal of Neuroscience 2I, 2939-2948.

Kandel, E. R., Schwartz, J. H., and Jessell, T. M. (2000). Principles of Neural

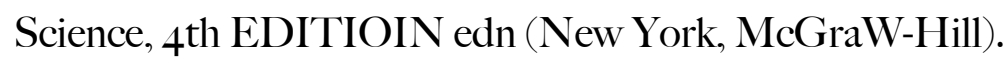

Koushika, S. P., Richmond, J. E., Hadwiger, G., Weimer, R. M., Jorgensen, E. M., and Nonet, M. L. (200I). A post-docking role for active zone protein Rim.[see comment]. Nature Neuroscience 4, 997-IO05.

Lin, R. C., and Scheller, R. H. (I997). Structural organization of the synaptic exocytosis core complex. Neuron 19, I087-I094.

Lu, Q., AtKisson, M. S., Jarvis, S. E., Feng, Z. P., Zamponi, G. W., and Dunlap, K. (200I). Syntaxin IA supports voltage-dependent inhibition of alpharB $\mathrm{Ca} 2+$ channels by Gbetagamma in chick sensory neurons. Journal of Neuroscience 2I, 2949-2957.

Margittai, M., Fasshauer, D., Jahn, R., and Langen, R. (2003a). The Habc domain and the SNARE core complex are connected by a highly flexible linker. Biochemistry 42, 4009-40I4.

Margittai, M., Widengren, J., Schweinberger, E., Schroder, G. F., Felekyan, S., Haustein, E., Konig, M., Fasshauer, D., Grubmuller, H., Jahn, R., and Seidel, C. A. (2003b). Single-molecule fluorescence resonance energy 
transfer reveals a dynamic equilibrium between closed and open conformations of syntaxin I. Proc Natl Acad Sci U S A IOO, I55'6-I552I.

Maruyama, I. N., and Brenner, S. (I99I). A phorbol ester/diacylglycerol-binding protein encoded by the unc-I3 gene of Caenorhabditis elegans. Proc Natl Acad Sci U S A 88, 5729-5733.

Misura, K. M., Scheller, R. H., and Weis, W. I. (2000). Three-dimensional structure of the neuronal-Seci-syntaxin Ia complex.[see comment]. Nature $404,355-362$.

Morihara, T., Mizoguchi, A., Takahashi, M., Kozaki, S., Tsujihara, T., Kawano, S., Shirasu, M., Ohmukai, T., Kitada, M., Kimura, K., et al. (I999). Distribution of synaptosomal-associated protein 25 in nerve growth cones and reduction of neurite outgrowth by botulinum neurotoxin A without altering growth cone morphology in dorsal root ganglion neurons and PCI2 cells. Neuroscience 9I, 695-706.

Munson, M., and Hughson, F. M. (2002). Conformational regulation of SNARE assembly and disassembly in vivo. J Biol Chem 277, 9375-938I.

Murthy, V. N., and Stevens, C. F. (I999). Reversal of synaptic vesicle docking at central synapses. Nat Neurosci 2, 5०3-5०7.

Nagamatsu, S., Fujiwara, T., Nakamichi, Y., Watanabe, T., Katahira, H., Sawa, H., and Akagawa, K. (1996). Expression and functional role of syntaxin I/HPC-I in pancreatic beta cells. Syntaxin IA, but not IB, plays a negative role in regulatory insulin release pathway. Journal of Biological Chemistry 27I, II6O-II65. 
Nonet, M. L., Saifee, O., Zhao, H., Rand, J. B., and Wei, L. (I998). Synaptic transmission deficits in Caenorhabditis elegans synaptobrevin mutants. Journal of Neuroscience $I 8,70-80$.

Novick, P., Field, C., and Schekman, R. (I980). Identification of 23 complementation groups required for post-translational events in the yeast secretory pathway. Cell 2I, 2O5-2I5.

Ohtsuka, T., Takao-Rikitsu, E., Inoue, E., Inoue, M., Takeuchi, M., Matsubara, K., Deguchi-Tawarada, M., Satoh, K., Morimoto, K., Nakanishi, H., and Takai, Y. (2002). Cast: a novel protein of the cytomatrix at the active zone of synapses that forms a ternary complex with RIMı and Munci3-I. J Cell Biol $158,577-590$.

Osen-Sand, A., Catsicas, M., Staple, J. K., Jones, K. A., Ayala, G., Knowles, J., Grenningloh, G., and Catsicas, S. (I993). Inhibition of axonal growth by SNAP-25 antisense oligonucleotides in vitro and in vivo. Nature $364,445^{-}$ 448 .

Osen-Sand, A., Staple, J. K., Naldi, E., Schiavo, G., Rossetto, O., Petitpierre, S., Malgaroli, A., Montecucco, C., and Catsicas, S. (1996). Common and distinct fusion proteins in axonal growth and transmitter release. J Comp Neurol 367, 222-234.

Otto, H., Hanson, P. I., and Jahn, R. (I997). Assembly and disassembly of a ternary complex of synaptobrevin, syntaxin, and SNAP-25 in the membrane of synaptic vesicles. Proceedings of the National Academy of Sciences of the United States of America 94, 6I97-620I. 
Pabst, S., Hazzard, J. W., Antonin, W., Südhof, T. C., Jahn, R., Rizo, J., and Fasshauer, D. (2000). Selective interaction of complexin with the neuronal SNARE complex. Determination of the binding regions. J Biol Chem 275, I9808-I98I8.

Perez-Branguli, F., Muhaisen, A., and Blasi, J. (2002). Munc I8a binding to syntaxin $\mathrm{IA}$ and $\mathrm{IB}$ isoforms defines its localization at the plasma membrane and blocks SNARE assembly in a three-hybrid system assay. Molecular \& Cellular Neurosciences 20, I69-I8O.

Perez-Branguli, F., Ruiz-Montasell, B., and Blasi, J. (1999). Differential interaction patterns in binding assays between recombinant syntaxin I and synaptobrevin isoforms. FEBS Letters $45^{8}, 60-64$.

Rao, S. S., Stewart, B. A., Rivlin, P. K., Vilinsky, I., Watson, B. O., Lang, C., Boulianne, G., Salpeter, M. M., and Deitcher, D. L. (200I). Two distinct effects on neurotransmission in a temperature-sensitive SNAP-25 mutant. Embo J 20, 676I-677I.

Reim, K., Mansour, M., Varoqueaux, F., McMahon, H. T., Südhof, T. C., Brose, N., and Rosenmund, C. (200I). Complexins regulate a late step in Ca2+dependent neurotransmitter release. Cell IO4, 7I-8I.

Rhee, J. S., Betz, A., Pyott, S., Reim, K., Varoqueaux, F., Augustin, I., Hesse, D., Südhof, T. C., Takahashi, M., Rosenmund, C., and Brose, N. (2002). Beta phorbol ester- and diacylglycerol-induced augmentation of transmitter release is mediated by Muncizs and not by PKCs. Cell IO8, I2II33. 
Richmond, J. E., Weimer, R. M., and Jorgensen, E. M. (200I). An open form of syntaxin bypasses the requirement for $\mathrm{UNC}-\mathrm{I} 3$ in vesicle priming. Nature 4I2, 338-34I.

Rizo, J., and Südhof, T. C. (2002). Snares and Muncı8 in synaptic vesicle fusion. Nat Rev Neurosci 3, 64I-653.

Rosenmund, C., Clements, J. D., and Westbrook, G. L. (1993). Nonuniform probability of glutamate release at a hippocampal synapse. Science 262 , $754-757$.

Rosenmund, C., Rettig, J., and Brose, N. (2003). Molecular mechanisms of active zone function. Curr Opin Neurobiol 13, 509-519.

Rosenmund, C., Sigler, A., Augustin, I., Reim, K., Brose, N., and Rhee, J. S. (2002). Differential control of vesicle priming and short-term plasticity by Munci3 isoforms. Neuron 33, 4II-424.

Rosenmund, C., and Stevens, C. F. (1996). Definition of the readily releasable pool of vesicles at hippocampal synapses. Neuron I6, II97-I207.

Ruiz-Montasell, B., Aguado, F., Majo, G., Chapman, E. R., Canals, J. M., Marsal, J., and Blasi, J. (1996). Differential distribution of syntaxin isoforms IA and IB in the rat central nervous system. European Journal of Neuroscience 8 , $2544-255^{2}$.

Sassa, T., Harada, S., Ogawa, H., Rand, J. B., Maruyama, I. N., and Hosono, R. (1999). Regulation of the UNC-I8-Caenorhabditis elegans syntaxin complex by UNC-13. Journal of Neuroscience 19, 4772-4777. 
Schikorski, T., and Stevens, C. F. (1997). Quantitative ultrastructural analysis of hippocampal excitatory synapses. J Neurosci $17,5858-5867$.

Schluter, O. M., Schmitz, F., Jahn, R., Rosenmund, C., and Südhof, T. C. (2004). A complete genetic analysis of neuronal Rab3 function. J Neurosci 24, $6629-6637$.

Schneggenburger, R., Sakaba, T., and Neher, E. (2002). Vesicle pools and shortterm synaptic depression: lessons from a large synapse. Trends Neurosci $25,206-212$.

Schoch, S., Castillo, P. E., Jo, T., Mukherjee, K., Geppert, M., Wang, Y., Schmitz, F., Malenka, R. C., and Südhof, T. C. (2002). RIMialpha forms a protein scaffold for regulating neurotransmitter release at the active zone. Nature $415,3^{2} \mathrm{I}-326$.

Schoch, S., Deak, F., Konigstorfer, A., Mozhayeva, M., Sara, Y., Südhof, T. C., and Kavalali, E. T. (200I). SNARE function analyzed in synaptobrevin/VAMP knockout mice. Science 294, III7-II22.

Schulze, K. L., Broadie, K., Perin, M. S., and Bellen, H. J. (I995). Genetic and electrophysiological studies of Drosophila syntaxin-IA demonstrate its role in nonneuronal secretion and neurotransmission. Cell $80,3 \mathrm{II}-320$.

Serra-Pages, C., Medley, Q. G., Tang, M., Hart, A., and Streuli, M. (I998). Liprins, a family of LAR transmembrane protein-tyrosine phosphatase-

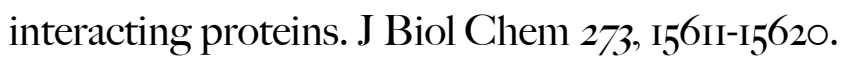


Siegel, G. J., Agranoff, B. W., Albers, R. W., Fisher, S. K., and Uhler, M. D. (I999). Basic Neurochemistry, Sixth Edition edn (Philadelphia, Lippincott Willians \& Wilkins).

Stanley, E. F. (2003). Syntaxin I modulation of presynaptic calcium channel inactivation revealed by botulinum toxin CI. European Journal of Neuroscience 17 , I3O3-I3O5.

Stanley, E. F., and Goping, G. (I99I). Characterization of a calcium current in a vertebrate cholinergic presynaptic nerve terminal. J Neurosci II, 985-993.

Stanley, E. F., and Mirotznik, R. R. (1997). Cleavage of syntaxin prevents G-protein regulation of presynaptic calcium channels. Nature $385,340-343$.

Stevens, C. F., and Sullivan, J. M. (I998). Regulation of the readily releasable vesicle pool by protein kinase C. Neuron 2I, 885-893.

Stevens, C. F., and Wesseling, J. F. (I999). Identification of a novel process limiting the rate of synaptic vesicle cycling at hippocampal synapses. Neuron 24 , IOI7-IO28.

Südhof, T. C. (2000). The synaptic vesicle cycle revisited. Neuron 28, 317-320.

Südhof, T. C., and Scheller, R. (200I). Mechanism and Regulation of Neurotransmitter Release. In Synapses, W. M. Cowan, T. C. Südhof, and C. F. Stevens, eds. (Baltimore, The Johns Hopkins University Press), pp. I77-2I5. 
Sutton, R. B., Fasshauer, D., Jahn, R., and Brunger, A. T. (I998). Crystal structure of a SNARE complex involved in synaptic exocytosis at $2.4 \mathrm{~A}$ resolution.[see comment]. Nature 395, 347-353.

Sweeney, S. T., Broadie, K., Keane, J., Niemann, H., and O'Kane, C. J. (I995). Targeted expression of tetanus toxin light chain in Drosophila specifically eliminates synaptic transmission and causes behavioral defects. Neuron I4, $34 \mathrm{I}-35^{\mathrm{I}}$

Takahashi, T., and Momiyama, A. (I993). Different types of calcium channels mediate central synaptic transmission. Nature $366,{ }_{5} 6-{ }_{5} 8$.

Teng, F. Y., Wang, Y., and Tang, B. L. (200I). The syntaxins. Genome Biology 2 , REVIEWS 3012.

Tucker, W. C., Weber, T., and Chapman, E. R. (2004). Reconstitution of Ca2+regulated membrane fusion by synaptotagmin and SNAREs. Science 304 , $435-438$.

Verhage, M., Maia, A. S., Plomp, J. J., Brussaard, A. B., Heeroma, J. H., Vermeer, H., Toonen, R. F., Hammer, R. E., van den Berg, T. K., Missler, M., et al. (2000). Synaptic assembly of the brain in the absence of neurotransmitter secretion. Science $287,864-869$.

Voets, T., Toonen, R. F., Brian, E. C., de Wit, H., Moser, T., Rettig, J., Südhof, T. C., Neher, E., and Verhage, M. (200I). Munci8-I promotes large densecore vesicle docking. Neuron 3 I, $5^{8 \mathrm{I}-59 \mathrm{I} \text {. }}$ 
Washbourne, P., Thompson, P. M., Carta, M., Costa, E. T., Mathews, J. R., Lopez-Bendito, G., Molnar, Z., Becher, M. W., Valenzuela, C. F., Partridge, L. D., and Wilson, M. C. (2002). Genetic ablation of the tSNARE SNAP-25 distinguishes mechanisms of neuroexocytosis. Nat Neurosci 5, I9-26.

Weber, T., Zemelman, B. V., McNew, J. A., Westermann, B., Gmachl, M., Parlati, F., Sollner, T. H., and Rothman, J. E. (I998). SNAREpins: minimal machinery for membrane fusion. Cell 92, 759-772.

Weimer, R. M., Richmond, J. E., Davis, W. S., Hadwiger, G., Nonet, M. L., and Jorgensen, E. M. (2003a). Defects in synaptic vesicle docking in unc-I8 mutants. Nature Neuroscience 6, IO23-IO3O.

Wheeler, D. B., Randall, A., and Tsien, R. W. (I994). Roles of N-type and Q-type $\mathrm{Ca} 2+$ channels in supporting hippocampal synaptic transmission. Science 264, IO7-III.

Wiser, O., Bennett, M. K., and Atlas, D. (1996). Functional interaction of syntaxin and SNAP-25 with voltage-sensitive L- and N-type Ca2+ channels. EMBO Journal $15,4 \mathrm{IOO}-4 \mathrm{IIO}$.

Wu, M. N., Fergestad, T., Lloyd, T. E., He, Y., Broadie, K., and Bellen, H. J. (I999). Syntaxin IA interacts with multiple exocytic proteins to regulate neurotransmitter release in vivo. [erratum appears in Neuron 2000 Mar;25(3):735]. Neuron 23, 593-605.

Yang, B., Steegmaier, M., Gonzalez, L. C., Jr., and Scheller, R. H. (2000). nSecI binds a closed conformation of syntaxiniA. Journal of Cell Biology I 4 , $247^{-2} 5^{2}$ 
Yoshihara, M., and Littleton, J. T. (2002). Synaptotagmin I functions as a calcium sensor to synchronize neurotransmitter release. Neuron 36, 897-908.

Zamponi, G. W. (2003). Regulation of presynaptic calcium channels by synaptic proteins. Journal of Pharmacological Sciences 92, 79-83.

Zhen, M., and Jin, Y. (I999). The liprin protein SYD-2 regulates the differentiation of presynaptic termini in C. elegans. Nature 4OI, 37I-375.

Zucker, R. S., and Regehr, W. G. (2002). Short-term synaptic plasticity. Annu Rev Physiol 64, 355-405. 


\section{Acknowledgements:}

I would like to thank all of the members of department of Membrane Biophysics for their scientific and professional support. I am extremely grateful to Professor Erwin Neher for being an exceptional model of my life as a scientist and for his constant support I am also indeed grateful for Professor Christian Rosenmund for their excellent guidance and encouragement.

Foremost, I acknowledge my past and present lab mates for their cooperative help and friendship. Especially, I am thankful for Dr. Alexander Meyer who provided me with brilliant suggestions and criticisms of my research. I wish to thank René Wiese, Albrecht Sigler, Susann Kaufmann and Michael Mansour for their sincere friendship and kind help for my German life. Also, I am more than happy to be with Jayeeta Basu, Mingshan Xue, Liyi Li, Dr. Jeong-Seop Rhee and Dr.Shutaro Katsurabayashi at our new lab in Houston. I cannot forget technical and administrative support of Ina Herfort, Ralf Nehering and Irmgard Barteczko.

I would also like to take this opportunity to thank my parents. Their endless love and belief created an environment that spurred my curiosity for research.

I dedicate this dissertation to my wife, Jiwon. 


\section{RAH, JONG-CHEOL}

$\begin{array}{ll}\text { Mai } 7 \text { I974 } & \begin{array}{l}\text { Geboren in Seoul, Korea } \\ \text { Sohn von Choong-Kyun Rah MBA und Choon-Hee Ban BSc. }\end{array} \\ \begin{array}{l}\text { Februar I993 - } \\ \text { Februar I999 }\end{array} & \begin{array}{l}\text { BSc. (Biologie/ Biochemie) an der Sogang University, Seoul, } \\ \text { Korea }\end{array} \\ \begin{array}{l}\text { Juli I995 - } \\ \text { Dezember I996 }\end{array} & \begin{array}{l}\text { Wehrdienst in der koreanischen Armee } \\ \text { September I999- } \\ \text { Februar 2000 }\end{array} \\ \begin{array}{l}\text { Austauschstudent am Imperial College of London, London, } \\ \text { Betreuung: Prof. Mustafa Djamgoz PhD }\end{array} \\ \begin{array}{l}\text { Maerz 2000 - } \\ \text { Februar 2002 }\end{array} \\ \begin{array}{l}\text { Korea } \\ \text { Betreuung: Prof.Yoo-Hun Suh, MD. PhD. }\end{array} \\ \begin{array}{l}\text { Austauschstudent am Imperial College of London, London, } \\ \text { Oktober 200I - } \\ \text { Februar 2002 }\end{array} \\ \begin{array}{l}\text { UK } \\ \text { Betreuung: Prof. Mustafa Djamgoz PhD }\end{array} \\ \begin{array}{l}\text { Dissertation in der Abteilung Membranbiophysik, } \\ \text { Arbeitsgruppe PD Dr.Christian Rosenmund, am Max-Planck- } \\ \text { Institut für biophysikalische Chemie, Göttingen, unter } \\ \text { Betreuung von PD Dr. Christian Rosenmund und Prof. Dr. } \\ \text { Erwin Neher }\end{array}\end{array}$

\section{Publikationen}

Jong-Cheol Rah, Stefan Gerber, Alexander Meyer, Jeong-Seop Rhee, Thomas Sudhof, Christian Rosenmund, Constitutively open conformation of syntaxin IB enhances vesicle priming and membrane exocytosis (in Vorbereitung)

Jong-Cheol Rah, Hye-Sun Kim, Sung Su Kim, Jae-Hyung Bach, Sung-Jin Jeong, JiHeui Seo, Cheol Hyoung Park, Yong-Sik Kim, and Yoo-Hun Suh, Effects of the carboxyl-terminal fragment of Alzheimer's amyloid precursor protein and amyloid bpeptide on the production of cytokines and nitric oxide in glial cells. FASEB J. $200 I$ Jun I5(8): $1463-1465$.

JH Kim, Jong-Cheol Rah, SP Fraser, KA Chang, MB Djamgoz, YH Suh Carboxyl-terminal peptide of beta-amyloid precursor protein blocks inositol I,4,5- 
trisphosphate-sensitive Ca2 + release in Xenopus laevis oocytes.. J Biol Chem. 2002 Jun $7 ; 277(23): 20256-63$.

JH Seo, Jong-Cheol Rah, SH Choi, JK Shin, K Min, HS Kim, CH Park, S Kim, EM Kim, SH Lee, S Lee, SW Suh, YH Suh a-Synuclein regulates neuronal survival via Bcl-2 family expression and $\mathrm{PI}_{3} /$ Akt kinase pathway. FASEB J. $2002 \mathrm{Nov}$; I6(I3):I826-I826. Epub 2002 Sep 05.

CH Park, SH Choi, CH Kim, JH Seo, JW Koo, Jong-Cheol Rah, HS Kim, SJ Jeong, WS Joo, YJ Lee, YS Kim, MS Kim, YH Suh. General pharmacology of indole $\left[2^{\prime}, 3^{\prime}: 3,4\right]$ pyrido[2,I-b]quinazolinium-5,7,8, I3-tetrahydro-I4-methyl-5-oxochloride, a new anti-dementia agent. Arzneimittelforschung. 2003;53(6):393-4OI.

JH Bach, HS Chae, Jong-Cheol Rah, MW Lee, SH Choi, JK Choi, YS Kim, KY Kim, WB Lee, YH Suh and SS Kim:C-terminal fragment of Amyloid Precursor Protein induces Astrocytosis J. Neurochem 200I Jul; 78(I):IO9-20

JH Seo, SH Kim, HS Kim, CH Park, SJ Jeong, JH Lee, SH Choi, KA Chang, Jong-Cheol Rah, JW Koo, EM Kim and YH Suh. Effects of Nicotine on APP Secretion and Ab- or CTIO5-Induced Toxicity 200I Biol.Psychiatry 200 I Feb I; 49(3):240-7

HS Kim, CH Park, SH Cha, JH Lee, SW Lee, YM Kim, Jong-Cheol Rah, SJ Jeong and YH Suh, C-terminal fragment of Alzheimer's APP destabilize calcium homeostasis and renders neuronal cells vulnerable to excitotoxicity. FASEB J. 2000 Aug;I4(II):I5O8-I7.

SJ Jeong, HS Kim, CH Park, KA Chang, DH Geum, CH Pak, JH Seo, JongCheol Rah, JH Lee, SH Choi, SG Lee and YH Suh, Subcellular localization of presenilins during mouse preimplantation development. FASEB J. $2000 \mathrm{Nov}$; I4 (I4): $217 I-2 I 76$.

CH Park, SH Choi, Y Piao, SH Kim, YJ Lee, HS Kim, SJ Jeong, Jong-Cheol Rah, JH Seo, KA Chang, YJ Jung and YH Suh. Glutamate and aspartate impair memory retenion and damage hypothalamic neurons in adult mice. Toxicol Lett. 2000 May I9; $I I 5(2): 117-25$

YH Suh, HS Kim, JP Lee, CH Park, SJ Jeong, SS Kim, Jong-Cheol Rah, JH Seo, SS Kim. Roles of A beta and carboxyl terminal peptide fragments of amyloid precursor protein in Alzheimer Disease. J Neural Transm Suppl 2000;(58): 65-82.

\section{(Buchkapitel)}

YH Suh, HS Kim, CH Park, JH Seo, JP Lee, SJ Jeong, SS Kim, JH Lee, SH Choi, KA Chang, Jong-Cheol Rah, SS Kim. Etiological roles of Ab and carboxyl terminal peptide fragments of amyloid precursor protein in Alzheimer disease.

Neuroscientific Basis of Dementia.

Goettingen, I7.09.2004 